KUL-TF-06/34

IFIC/06-29

FTUV-06/1216

hep-th/0612210

\title{
Special geometry for arbitrary signatures
}

\author{
María A. Lledół, Óscar Maciáł, Antoine Van Proeyen ${ }^{b}$ and Veeravalli S. Varadarajan ${ }^{\natural}$ \\ \#Departament de Física Teòrica, Universitat de València and IFIC, \\ C/ Dr. Moliner, 50, E-46100 Burjassot (València), Spain. \\ ' Instituut voor Theoretische Fysica, Katholieke Universiteit Leuven. \\ Celestijnenlaan 200D B-3001 Leuven, Belgium. \\ ${ }^{\natural}$ Department of Mathematics, UCLA. Los Angeles, CA 90095-1555, USA \\ E-mail: Maria.Lledo@ific.uv.es, Oscar.Macia@ific.uv.es, \\ Antoine.VanProeyen@fys.kuleuven.be,vsv@math.ucla.edu
}

\begin{abstract}
In this paper we generalize special geometry to arbitrary signatures in target space. We formulate the definitions in a precise mathematical setting and give a translation to the coordinate formalism used in physics. For the projective case, we first discuss in detail projective Kähler manifolds, appearing in $N=1$ supergravity. We develop a new point of view based on the intrinsic construction of the line bundle. The topological properties are then derived and the Levi-Civita connection in the projective manifold is obtained as a particular projection of a Levi-Civita connection in a 'mother' manifold with one extra complex dimension. The origin of this approach is in the superconformal formalism of physics, which is also explained in detail. Finally, we specialize these results to projective special Kähler manifolds and provide explicit examples with different choices of signature.
\end{abstract}

Contribution to the handbook on pseudo-Riemannian geometry and supersymmetry, ed. V. Cortés, published by the European Mathematical Society in the series "IRMA Lectures in Mathematics and Theoretical Physics". 


\section{Contents}

\begin{tabular}{lll}
\hline 1 & Introduction & 3 \\
\hline
\end{tabular}

2 Kähler manifolds $\quad \mathbf{6}$

$2.1 \quad$ Generalities on quasicomplex and complex manifolds . . . . . 7

2.2 Hermitian metrics and Kähler metrics . . . . . . . . . . . . . . 99 9

2.3 Hermitian line bundles and fiber metrics . . . . . . . . . . . . 15

3 Rigid special Kähler manifolds $\quad 18$

3.1 Some geometric preliminaries . . . . . . . . . . . . . . . 18

3.2 Definition of rigid special Kähler manifolds . . . . . . . . . . . 19

3.3 The signature of the metric . . . . . . . . . . . . . . . . . 24

3.4 The prepotential . . . . . . . . . . . . . . . . . . . . . . . 28

3.5 The pseudo-Kähler case . . . . . . . . . . . . . . . . . . 32

3.6 A special pseudo-Kähler manifold . . . . . . . . . . . . . . . . 33

3.7 The holomorphic cubic form . . . . . . . . . . . . . . . . 35

4 Projective Kähler (Kähler-Hodge) manifolds 38

4.1 Affine transformations, isometries and homothetic Killing vec-

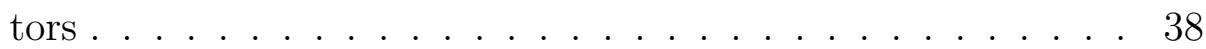

4.2 Definition of projective Kähler manifolds . . . . . . . . . . . . 44

4.3 The Levi-Civita connection on a Kähler-Hodge manifold . . . 51

4.4 Examples of Kähler-Hodge manifolds . . . . . . . . . . . . . . 56

$\begin{array}{llr}5 & \text { Conformal calculus } & 59\end{array}$

5.1 Real manifold. . . . . . . . . . . . . . . . . . . . . . 59

5.2 Kähler manifolds . . . . . . . . . . . . . . . . . . . . . 61

$\begin{array}{|ll|}6 & \text { Projective special Kähler manifolds }\end{array}$

6.1 Definition of projective special Kähler manifolds . . . . . . . . 65

6.2 Examples of projective special Kähler manifolds . . . . . . . . 68

\begin{tabular}{lll|}
\hline 7 & Conclusions & 73 \\
\hline
\end{tabular}

\begin{tabular}{ll}
\hline A Some technical results & 75
\end{tabular}

\begin{tabular}{|lll}
\hline B Connection on a principal bundle and covariant derivative & 77
\end{tabular} 


\section{Introduction}

Special Kähler geometry is the geometry of the manifold spanned by the scalars of vector multiplets of $D=4, N=2$ supersymmetry. The rigid version, that occurs in supersymmetry without gravity, appeared first in Refs. [1, 2]. The construction for $N=2$ supergravity appeared in Ref. 3], and it is called projective special Kähler geometry. It played an important role in several developments of string theory.

These first formulations offered a local point of view. For the rigid case, the condition for a Kähler geometry to be 'special' is the existence of a preferred set of holomorphic coordinates $z^{i}$, called special coordinates in which the Kähler potential $\mathcal{K}$ can be expressed in terms of a holomorphic function, the prepotential $\mathcal{F}$,

$$
g_{i \bar{\jmath}}=\frac{\partial \mathcal{K}}{\partial z^{i} \partial \bar{z}^{\bar{\jmath}}}, \quad \mathcal{K}=2 \Im\left(\frac{\partial \mathcal{F}}{\partial z^{k}} \bar{z}^{\bar{k}}\right) .
$$

It is seen then as a further restriction on the metric, compatible with the complex structure. For the projective case, the original construction was based on superconformal tensor calculus and involves a projectivization of the manifold due to the extra vector field, the graviphoton, which does not have associated a scalar. In simple words, one has a rigid special manifold with a dilation symmetry and a non physical scalar, which is projected out by fixing the symmetry.

The property of being a special Kähler manifold is then a purely geometrical one, and can be formulated independently of supersymmetry. It is given though in terms of a preferred set of coordinates. Although this local formulation is not incomplete (there has to exists an open cover of the manifold by special coordinates), it remains the intriguing question if there is a way of defining what is a special Kähler manifold with global statements, independent of coordinates. The first attempts were in Refs. [4, 5, 6]. A set of equivalent definitions was found in Ref. [7], and later on, a mathematical formulation appeared in Ref. [8].

One fundamental ingredient in the global approach is the existence of a certain flat symplectic bundle. Peculiar to Freed's formulation [8] is that the symplectic bundle is recognised as the tangent bundle, so the construction is intrinsic. In fact, the rigid case (see Definition 3.1) comes out very elegantly, and for this part we will follow closely Freed's work (with the exception of the pseudo-Riemannian case, which we will mention later). The projective 
case is much more involved. We define a projective special manifold in terms of a rigid special manifold with a homothetic Killing vector (see Definition 4.2). In this way, the definition is not only intrinsic but directly related to the way in which it is obtained in supergravity [3]. The point of contact of this definition with Freed's work is in his Proposition 4.6.

So far as for Riemannian, special Kähler manifolds. Pseudo-Riemannian special Kähler manifolds ${ }^{1}$ are very relevant in supergravity. A physically sensible supergravity theory must have a positive definite target-space metric. From the conformal calculus approach it is known that in order to get such positive definite metric the rigid Kähler manifold before projection has to signature $(2,2 n)$. But pseudo-Riemannian special Kähler manifolds present an additional complication. Special coordinates are complex coordinates constructed from a set of flat Darboux coordinates $\left(q^{i}, p_{i}\right)$ by taking the holomorphic extension of the $q^{i}$ s (or, alternatively, of the $p_{i}^{\prime} s$ ). They have then the prepotential property (1). When the signature of the metric is indefinite, this holomorphic extension does not always result in a set of $n$ independent holomorphic coordinates. There is a subclass of Darboux systems that have this property. It is important thought that one can always make a constant symplectic rotation to coordinates $\left(q^{\prime i}, p_{i}^{\prime}\right)$ such that the $q^{i}$ 's extend to special coordinates, so there is still a covering of the manifold by special coordinates. But not all flat Darboux systems are suitable to obtain special coordinates. As a consequence, the structure group of the bundle is reduced to a subgroup of the symplectic group. This was first observed in [12]. Nevertheless, flat Darboux coordinates which do not lead to special coordinates nor prepotential are very relevant. They were used to prove that one can break $N=2$ supersymmetry partially to $N=1[13$ and not necessarily to $N=0$, as it was thought before. This is an extremely important property for phenomenological applications. It is then one of the main motives of this work (which was missing in Ref. [8]) to generalize the construction of special geometry to arbitrary signatures.

In another context, it has been recently shown [14, 15, 16] that relating flat Darboux coordinates with the real central charges and attractor equations

\footnotetext{
${ }^{1}$ Note that all discussions on the signature in this work concern the signature of the Kähler manifold, i.e. the target manifold of the supergravity theory. This is unrelated to the signature of spacetime, which we keep Minkowskian to have the standard special geometries. Discussions on generalizations to Euclidean spacetime signature are in Refs. [9, 10, 11].
} 
would have a simplifying role in the description of the attractor mechanism of black holes in $N=2$ supergravity (see Refs. [17, 18, 19] for the attractor mechanism).

Pseudo-Riemannian, projective special Kähler manifolds appear also as dimensional reductions of supergravity theories in eleven dimensions and exotic signatures, obtained by duality transformations from the standard Minkowskian signature. These are the theories $M^{*}$ and $M^{\prime}$, in signatures $(9,2)$ and $(6,5)$ proposed in Ref. [20]. The pseudo-Riemannian special manifolds arising in $D=4$ are discussed in Ref. [21].

An important part of the work in dealing with projective special Kähler manifolds concerns in fact a more general class of Kähler manifolds, the socalled Kähler-Hodge manifolds. ${ }^{2}$ It was found in Ref. [22] that the Kähler geometries of $N=1$ supergravity should be Kähler-Hodge. We propose an intrinsic definition of projective Kähler manifolds (see Definition 4.2), inspired in the conformal calculus approach used in physics. Then we show that they have integer Kähler cohomology class, so they are Kähler-Hodge.

The paper is as much self contained as possible, so we have included vast review material. On the other hand, having in mind the connection to physics, we have tried to work everything out in coordinates, as to have the sometimes difficult translation between two languages, the physicist's and the mathematician's one, each of them with its own advantages. We have also taken time in explaining some examples, which may clarify the abstract definitions.

The paper is organized as follows.

In Section 2 we review basic material on complex and Kähler manifolds and Hermitian bundles. It is used extensively in the paper, so with it we set the basic notation. The reader can also skip it and come back to it punctually when some concept is called for.

Section 3 is devoted to the rigid case. We start with some geometric preliminaries not included in Section 2 and then we take up the definition of rigid special Kähler manifolds. We follow the lines of Ref. [8], explaining carefully how the formulas in coordinates are obtained from the abstract definition. Then we treat the pseudo-Riemannian case, giving some clarifying

\footnotetext{
${ }^{2}$ A Kähler-Hodge manifold is a Kähler manifold with integer Kähler cohomology class.
} 
simple examples. We come back to Freed's treatment for the holomorphic cubic form, which will be used later on.

Section 4 is dedicated to projective Kähler manifolds as a previous step towards projective special geometry. We introduce some concepts on affine transformations and homothetic Killing vectors and derive some of its properties. This material is not new, but perhaps not so widely known, so it is fundamental to have it at hand. Then we define projective Kähler manifolds starting from a Kähler manifold $\tilde{\mathcal{M}}$ (with arbitrary signature) which has an action of $\mathbb{C}^{\times}$(as well as other properties, see Definition 4.2). We then consider $\mathcal{M}=\tilde{\mathcal{M}} / \mathbb{C}^{\times}$and construct the symplectic and the line bundles over it. The line bundle has a Hermitian metric induced from the Kähler metric on $\tilde{\mathcal{M}}$ (here the importance of the intrinsic approach), whose Ricci form turns out to be closed and non degenerate, defining then a symplectic structure (actually, a Kähler one). Since it is the first Chern class of a line bundle, the manifold is Kähler-Hodge.

We then propose an alternative and beautiful way of understanding the Levi-Civita connection in $\mathcal{M}$, directly induced from the one in $\tilde{\mathcal{M}}$. It is a bit involved, but it really gives precious insight into the geometry of $\mathcal{M}$.

Section 5 is a brief excursion on the origin of projective Kähler geometry as it is seen from a model in physics. It is the simplest one to consider, and it does not include supersymmetry. Indeed, the ideas of conformal calculus are more general than their applications to supergravity.

In Section 6 we impose on $\tilde{\mathcal{M}}$ the condition to be rigid special Kähler, then $\mathcal{M}$ will be a projective special Kähler manifold. The precise definition is Definition 6.1, and the consequences are analysed in the sequel. In particular, we obtain the holomorphic cubic form and then the formula for the curvature. We conclude with some examples, in particular the pseudo-Riemannian space

$$
\frac{\mathrm{SU}(1,2)}{\mathrm{SU}(1,1) \times \mathrm{U}(1)} \text {. }
$$

\section{Kähler manifolds}

This first section recapitulates the basic definitions on complex manifolds and Kähler manifolds in particular. It is essentially a summary of part of Chapter IX in Ref. [23]. It can be skipped by readers familiar with Kähler manifolds or used just to set the conventions. 


\subsection{Generalities on quasicomplex and complex mani- folds}

Let $\mathcal{M}$ be a quasicomplex (or almost complex) manifold of dimension $2 n$, with $J: T \mathcal{M} \mapsto T \mathcal{M}$ the quasicomplex structure, $J^{2}=-1$.

\section{Remark 2.1}

Suppose that $\mathcal{M}$ is a complex manifold and that $\left(z^{1}, \ldots z^{n}\right)$ are complex coordinates on an open set $U \subset \mathcal{M}, z^{j}=x^{j}+\mathrm{i} y^{j}$. Then, $\left(x^{1}, \ldots x^{n}, y^{1}, \ldots y^{n}\right)$ is a coordinate system in $U$ and we have that

$$
J\left(\frac{\partial}{\partial x^{j}}\right)=\frac{\partial}{\partial y^{j}}, \quad J\left(\frac{\partial}{\partial y^{j}}\right)=-\frac{\partial}{\partial x^{j}}, \quad j=1, \ldots n .
$$

Let $T_{m}^{c} \mathcal{M}$ denote the complexification of the tangent space at $m \in \mathcal{M}$. We denote by $T_{m}^{1,0} \mathcal{M}$ and $T_{m}^{0,1} \mathcal{M}$ the eigenspaces of $J$ at $m$ with eigenvalues $\mathrm{i}$ and $-\mathrm{i}$ respectively. Then

$$
Z=X-\mathrm{i} J X \in T_{m}^{1,0} \mathcal{M}, \quad \bar{Z}=X+\mathrm{i} J X \in T_{m}^{0,1} \mathcal{M}
$$

for any real vector $X \in T_{m} \mathcal{M}$. The operation $Z \mapsto \bar{Z}$ is a real linear endomorphism called complex conjugation. From now on we will denote the (complexified) tangent space simply as $T_{m}=T_{m} \mathcal{M}$.

Let $T_{m}^{* c}$ denote the complexification of the cotangent space at $m$ and $T^{* c}$ the complexified cotangent bundle of $\mathcal{M}$. Let $\omega \in T^{*}$. The pull back, at each point $m$, of $\omega$ through $J$,

$$
J^{*} \omega_{m}(X)=\omega_{m}(J X), \quad \forall X \in T_{m}
$$

defines an endomorphism

$$
J^{*}: T^{*} \rightarrow T^{*}
$$

with $\left(J^{*}\right)^{2}=-1$, which extends in the obvious way to the complexified cotangent space. The eigenspaces of eigenvalues $\mathrm{i}$ and $-\mathrm{i}$ of $J^{*}$ at $m$ are denoted as

$$
\Omega_{m}^{1,0}=\Lambda^{1,0} T_{m}^{* c} \quad \text { and } \quad \Omega_{m}^{0,1}=\Lambda^{0,1} T_{m}^{* c}
$$


respectively. One has that

$$
\begin{array}{ll}
\Omega_{m}^{0,1}=\left\{\omega_{m} \in T_{m}^{* c} / \omega_{m}(Z)=0\right. & \left.\forall Z \in T_{m}^{1,0}\right\} \\
\Omega_{m}^{1,0}=\left\{\omega_{m} \in T_{m}^{* c} / \omega_{m}(Z)=0\right. & \left.\forall Z \in T_{m}^{0,1}\right\}
\end{array}
$$

Since the exterior product space, $\Omega_{m}=\sum_{r=0}^{2 n} \Lambda^{r} T_{m}^{* c}$, is generated by $\Omega_{m}^{0,0}, \Omega_{m}^{1,0}$ and $\Omega_{m}^{0,1}, \Omega_{m}$ has a bigrading

$$
\Omega_{m}=\sum_{p, q=0}^{n} \Omega_{m}^{p, q},
$$

and so has the space of complex forms

$$
\Omega=\sum_{p, q=0}^{n} \Omega^{p, q} .
$$

\section{Remark 2.2}

If $\mathcal{M}$ is a complex manifold, as in Remark 2.1, then

$$
\begin{aligned}
& T_{m}^{1,0}=\operatorname{span}_{\mathbb{C}}\left\{\left.\frac{\partial}{\partial z^{j}}\right|_{m}=\left.\frac{1}{2}\left(\frac{\partial}{\partial x^{j}}-\mathrm{i} \frac{\partial}{\partial y^{j}}\right)\right|_{m}\right\}_{j=1}^{n}, \\
& T_{m}^{0,1}=\operatorname{span}_{\mathbb{C}}\left\{\left.\frac{\partial}{\partial \bar{z}^{j}}\right|_{m}=\left.\frac{1}{2}\left(\frac{\partial}{\partial x^{j}}+\mathrm{i} \frac{\partial}{\partial y^{j}}\right)\right|_{m}\right\}_{j=1}^{n} .
\end{aligned}
$$

For the complex forms we have

$$
\begin{aligned}
& \Omega_{m}^{1,0}=\operatorname{span}_{\mathbb{C}}\left\{\left.\mathrm{d} z^{j}\right|_{m}=\left.\left(\mathrm{d} x^{j}+\mathrm{id} y^{j}\right)\right|_{m}\right\}_{j=1}^{n}, \\
& \Omega_{m}^{0,1}=\operatorname{span}_{\mathbb{C}}\left\{\left.\mathrm{d} \bar{z}^{\bar{\jmath}}\right|_{m}=\left.\left(\mathrm{d} x^{j}-\mathrm{id} y^{j}\right)\right|_{m}\right\}_{j=1}^{n} .
\end{aligned}
$$

The set of forms

$$
\begin{array}{r}
\left\{\mathrm{d} z^{j_{1}} \wedge \mathrm{d} z^{j_{2}} \wedge \ldots \mathrm{d} z^{j_{p}} \wedge \mathrm{d} \bar{z}^{\bar{k}_{1}} \wedge \mathrm{d} \bar{z}^{\bar{k}_{2}} \wedge \ldots \mathrm{d} \bar{z}^{\bar{k}_{q}}\right\} \\
1 \leq j_{1} \leq j_{2} \leq \ldots j_{p} \leq n, \quad 1 \leq \bar{k}_{1} \leq \bar{k}_{2} \leq \ldots \bar{k}_{q} \leq n
\end{array}
$$

is a local basis of $\Omega^{p, q}$.

For a complex manifold one can prove [23] that the differential

$$
\mathrm{d} \Omega^{p, q} \subset \Omega^{p+1, q}+\Omega^{p, q+1} .
$$


Then we can define $\partial: \Omega^{p, q} \rightarrow \Omega^{p+1, q}$ and $\bar{\partial}: \Omega^{p, q} \rightarrow \Omega^{p, q+1}$ as

$$
\mathrm{d}=\partial+\bar{\partial}
$$

and since $\mathrm{d}^{2}=0$ we have

$$
\partial^{2}=0, \quad \bar{\partial}^{2}=0, \quad \partial \circ \bar{\partial}+\bar{\partial} \circ \partial=0 .
$$

A form $\omega \in \Omega^{p, 0}$ is said to be holomorphic if $\bar{\partial} \omega=0$. A form $\omega \in \Omega^{0, p}$ is said to be antiholomorphic if $\partial \omega=0$. A function is holomorphic if

$$
\frac{\partial}{\partial \bar{z}^{j}} f=0, \quad j=1, \ldots n
$$

(respectively, antiholomorphic). A holomorphic vector field $Z$ is a complex vector field of type $(p, 0)$ such that $Z f$ is holomorphic for every holomorphic $f$. Locally,

$$
Z=\sum_{j=1}^{n} f^{j} \frac{\partial}{\partial z^{j}}
$$

with all the $f^{j}$ holomorphic.

\subsection{Hermitian metrics and Kähler metrics}

A Hermitian metric on a quasicomplex manifold $\mathcal{M}$ with quasicomplex structure $J$ is a Riemannian metric $g$ such that

$$
g(J X, J Y)=g(X, Y), \quad \forall X, Y \in T \mathcal{M} .
$$

Every paracompact quasicomplex manifold admits a Hermitian metric. This is because for a given Riemannian metric $h$ and a quasicomplex structure $J$ we can obtain a Hermitian metric by setting

$$
g(X, Y)=h(X, Y)+h(J X, J Y) \quad \forall X, Y \in T \mathcal{M},
$$

$g$ is extended to $T^{c}$ by linearity. It is easy to check that

1. $g(Z, W)=0$ for $Z, W$ of type $(1,0)$,

2. $g(Z, \bar{Z})>0$

3. $g(\bar{Z}, \bar{W})=\overline{g(Z, W)}$.

The fundamental 2-form of a Hermitian metric is

$$
\Phi(X, Y)=g(X, J Y) \quad \forall X, Y \in T \mathcal{M} .
$$

It is non degenerate at each point of the manifold. 
Remark 2.3 Almost complex linear connections

The torsion of a quasicomplex structure $J$ is the tensor field (1-covariant, 2-contravariant)

$$
N(X, Y)=2\{[J X, J Y]-[X, Y]-J[X, J Y]-J[J X, Y]\} .
$$

A quasicomplex structure is said to be integrable if it has no torsion. This is equivalent to saying that the commutator of two vector fields of type $(1,0)$ (alternatively $(0,1))$ is a vector field of type $(1,0)$ (alternatively $(0,1)$ ). To see this, let $Z, W$ be such that $J Z=\mathrm{i} Z$ and $J W=\mathrm{i} W$, then if $N(Z, W)=0$ it is immediate that $J[Z, W]=\mathrm{i}[Z, W]$. In the other direction, a real vector field can be always written as the sum $X=Z+\bar{Z}$, where $Z$ is $(1,0)$ and $\bar{Z}$ is $(0,1)$. Let also $Y=W+\bar{W}$. Then it is immediate to prove that $N(X, Y)=0$.

A quasicomplex structure is a complex structure if and only if it is integrable. This is the Newlander-Nirenberg theorem [24].

We say that a linear connection is quasicomplex if the covariant derivative of the quasicomplex structure is zero (which is equivalent to being a connection in the bundle of complex linear frames). Every quasicomplex manifold admits a quasicomplex affine connection whose torsion $T$ is proportional to the torsion $N$ of the quasicomplex structure.

In general, the Riemannian connection associated to a Hermitian metric is not quasicomplex. If it is so, then the quasicomplex structure has no torsion and the fundamental form is closed. The converse is also true: for a complex manifold, the Riemannian connection of a Hermitian metric is quasicomplex if and only if the fundamental 2 -form $\Phi$ is closed. (The proof of these statements can be read in Ref. [23], Chapter IX.)

A quasicomplex manifold, with a Hermitian metric is a quasi-Kähler (or almost Kähler) manifold if the fundamental form is closed.

Let $\mathcal{M}$ be a differential manifold. A symplectic structure on $\mathcal{M}$ is a 2-form $\Phi$ such that

i. It is closed, $\mathrm{d} \Phi=0$,

ii. It is non degenerate: for every $X \in T \mathcal{M}$, there exists $Y \in T \mathcal{M}$ such that $\Phi(X, Y) \neq 0$.

The couple $(\mathcal{M}, \Phi)$ is a symplectic manifold, and $\mathcal{M}$ has always even dimension. In any symplectic manifold, we have local Darboux coordinates defined by the following theorem. 
Theorem 2.4 (Darboux) If $\mathcal{M}$ is a symplectic manifold, $\operatorname{dim} \mathcal{M}=2 n$, for each $m \in \mathcal{M}$ there is a chart $(U, \varphi: U \rightarrow \mathbb{R})$ such that $\varphi(m)=0$ and for $u \in U, \varphi(u)=\left(x^{1}(u), \ldots x^{n}(u), y_{1}(u), \ldots y_{n}(u)\right)$ and $\Phi$ on the open set $U$ is

$$
\left.\Phi\right|_{U}=\sum_{i=1}^{n} \mathrm{~d} x^{i} \wedge \mathrm{d} y_{i}
$$

Notice that a quasi-Kähler manifold is a symplectic manifold, since the fundamental 2-form is non degenerate. If, in addition, the manifold is complex then it is a Kähler manifold. Moreover, let $D$ be a Riemannian connection, so $D_{X} g=0$ for every vector field $X$ on $\mathcal{M}$. We have that

$$
D_{X} \Phi\left(Y, Y^{\prime}\right)=D_{X} g\left(Y, J Y^{\prime}\right)+g\left(Y,\left(D_{X} J\right) Y^{\prime}\right)=0,
$$

which means that the Riemannian connection is trivially a symplectic connection.

The holonomy of a Kähler manifold of complex dimension $n$ is a subgroup of $\mathrm{U}(n) \simeq \mathrm{O}(2 n) \cap \mathrm{GL}(n, \mathbb{C})$, since the Riemannian connection is quasicomplex. Here $\mathrm{GL}(n, \mathbb{C})$ is taken in its real representation

$$
A+\mathrm{i} B \longrightarrow\left(\begin{array}{cc}
A & B \\
-B & A
\end{array}\right)
$$

One can prove that if the manifold is Ricci flat then the restricted holonomy group (that is, considering only parallel displacements along paths that are homotopic to a point) is contained in $\mathrm{SU}(n)$.

Kähler manifolds in coordinates. On a quasicomplex manifold, we can consider the principal bundle of unitary frames, that is the bundle of complex frames that are orthonormal with respect to the Hermitian metric. Its structural group is $\mathrm{U}(n)$. We will denote this bundle by $\mathrm{U}(\mathcal{M})$. We want to give the metric, connection and curvature of a Kähler manifold in coordinates.

Let $\mathcal{M}$ be a complex manifold, with Hermitian metric $g$ and complex structure $J$. We use the notation of Remarks 2.1, and 2.2 and denote

$$
Z_{i}=\frac{\partial}{\partial z^{i}}, \quad \bar{Z}_{i}=Z_{\bar{\imath}}=\frac{\partial}{\partial \bar{z}^{i}} .
$$


It is easy to see that

$$
g_{i j}=g\left(Z_{i}, Z_{j}\right)=0, \quad g_{\bar{\imath} \bar{\jmath}}=g\left(\bar{Z}_{i}, \bar{Z}_{j}\right)=0,
$$

and the only non zero components are of the form $g_{i \bar{\jmath}}=g\left(Z_{i}, \bar{Z}_{j}\right)=g_{\bar{\jmath} i}$, sq

$$
g=g_{i \bar{\jmath}}\left(\mathrm{d} z^{i} \otimes \mathrm{d} \bar{z}^{\bar{\jmath}}+\mathrm{d} \bar{z}^{\bar{\jmath}} \otimes \mathrm{d} z^{i}\right)=2 g_{i \bar{\jmath}} \mathrm{d} z^{i} \mathrm{~d} \bar{z}^{\bar{\jmath}} .
$$

Since $J\left(Z_{i}\right)=\mathrm{i} Z_{i}$ and $J\left(\bar{Z}_{\bar{\imath}}\right)=-\mathrm{i} \bar{Z}_{\bar{\imath}}$, the fundamental 2 -form is

$$
\Phi=-2 \mathrm{i} g_{i \bar{\jmath}} \mathrm{d} z^{i} \wedge \mathrm{d} \bar{z}^{\bar{\jmath}} .
$$

If $\mathcal{M}$ is a Kähler manifold, the fundamental form is closed, so

$$
\mathrm{d} \Phi=-2 \mathrm{i}\left(\frac{g_{i \bar{\jmath}}}{\partial z^{k}} \mathrm{~d} z^{k} \wedge \mathrm{d} z^{i} \wedge \mathrm{d} \bar{z}^{\bar{\jmath}}+\frac{g_{i \bar{\jmath}}}{\partial \bar{z}^{\bar{k}}} \mathrm{~d} \bar{z}^{\bar{k}} \wedge \mathrm{d} z^{i} \wedge \mathrm{d} \bar{z}^{\bar{\jmath}}\right)=0
$$

which implies

$$
\frac{g_{i \bar{\jmath}}}{\partial z^{k}}-\frac{g_{k \bar{\jmath}}}{\partial z^{i}}=0, \quad \frac{g_{i \bar{\jmath}}}{\partial \bar{z}^{\bar{k}}}-\frac{g_{i \bar{k}}}{\partial \bar{z}^{\bar{j}}}=0 .
$$

These equations are the integrability condition for the existence of a real valued function $\mathcal{K}$ such that

$$
g_{i \bar{\jmath}}=\frac{\partial \mathcal{K}}{\partial z^{i} \bar{z}^{\bar{\jmath}}}
$$

For any real function $\mathcal{K}$, the tensor $g_{i \bar{j}}$ satisfies $\left(g_{i \bar{j}}\right)^{*}=g_{j \bar{\imath}}$, and property (5). If it is positive definite, then it is a Kähler metric on $\mathcal{M}$. So any Kähler metric can be written locally in this way. Notice also that $\mathcal{K}$ is defined modulo a holomorphic function $f$,

$$
\mathcal{K} \rightarrow \mathcal{K}+f(z)+f(\bar{z}) .
$$

$\mathcal{K}$ is the Kähler potential

We will denote by $I$ an arbitrary index in $\{1,2, \ldots 2 n\}$ and by $\left\{x^{I}\right\}$ arbitrary coordinates in $\mathcal{M}$. Let $Y=Y^{I} \frac{\partial}{\partial x^{I}}=Y^{I} \partial_{I}$ be a vector field on $\mathcal{M}$. The covariant derivative of $Y$ with respect to a linear connection can be written as

$$
\left(D_{J} Y\right)^{I}=\frac{\partial Y^{I}}{\partial x^{J}}+\Gamma_{J K}^{I} Y^{K}
$$

\footnotetext{
${ }^{3}$ Note the conventions with factors for symmetric products and for forms. A symmetric product of forms $\alpha \beta$ is $\frac{1}{2}(\alpha \otimes \beta+\beta \otimes \alpha)$. Similarly a wedge product is taken as $\alpha \wedge \beta=$ $\frac{1}{2}(\alpha \otimes \beta-\beta \otimes \alpha)$.
} 
where the Christoffel symbols $\Gamma$ are

$$
\Gamma_{J K}^{I}=\left(D_{J} \partial_{K}\right)^{I} .
$$

The Levi-Civita connection is the only torsionfree connection satisfying $D g=$ 0. The Christoffel symbols are

$$
\Gamma_{J I}^{K}=\frac{1}{2} g^{K L}\left(\frac{\partial g_{L I}}{\partial x^{J}}+\frac{\partial g_{J L}}{\partial x^{I}}-\frac{\partial g_{J I}}{\partial x^{L}}\right) .
$$

For a complex manifold, we can extend the covariant differentiation to complex vector fields by linearity. We can then consider $I=i$ for $I=1, \ldots n$ and $I=n+\bar{\imath}$ for $I=n+1, \ldots 2 n$. The Christoffel symbols become complex, and it is easy to see that

$$
\bar{\Gamma}_{J K}^{I}=\Gamma_{\bar{J} \bar{K}}^{\bar{I}},
$$

where we have denoted $\bar{I}=I+n$ for $I=i$ and $\bar{I}=I-n$ for $I=n+i$.

A linear connection is quasicomplex if the complex structure is parallel. For $J=\mathrm{id} z^{j} \otimes \partial_{j}-\mathrm{id} \bar{z}^{\bar{\jmath}} \otimes \partial_{\bar{\jmath}}$ this means

$$
\left(D_{A} J\right)_{C}^{B}=\Gamma_{A D}^{B} J_{C}^{D}-\Gamma_{A C}^{D} J_{D}^{B}=0 \Rightarrow \Gamma_{A \bar{c}}^{d}=\Gamma_{A c}^{\bar{d}}=0 .
$$

If the connection is torsionfree we have,

$$
T(X, Y)=D_{X} Y-D_{Y} X-[X, Y]=0 \Rightarrow \Gamma_{B C}^{A}=\Gamma_{C B}^{A},
$$

so the only non zero Christoffel symbols of the quasicomplex connection are

$$
\Gamma_{j k}^{i}=\Gamma_{k j}^{i}, \quad \Gamma_{\bar{\jmath} \bar{k}}^{\bar{\imath}}=\Gamma_{\bar{k} \bar{j}}^{\bar{c}} .
$$

If the Riemannian connection is quasicomplex then the manifold is a Kähler manifold, and we have from (6) and (5)

$$
\Gamma_{j k}^{i}=g^{i \bar{\ell}} \partial_{j} g_{\bar{\ell} k}, \quad \Gamma_{\bar{\jmath} \bar{k}}^{\bar{\imath}}=g^{\bar{\imath} \ell} \partial_{\bar{\jmath}} g_{\ell \bar{k}} .
$$

The curvature tensor associated to a linear connection is a 3-contravariant 1-covariant tensor given by

$$
R(X, Y) Z=\left[D_{X}, D_{Y}\right] Z-D_{[X, Y]} Z, \quad X, Y, Z \in T \mathcal{M}
$$

and in components

$$
R_{J K L}^{I}=\left(\frac{\partial \Gamma_{L J}^{I}}{\partial x^{K}}-\frac{\partial \Gamma_{K J}^{I}}{\partial x^{L}}\right)+\sum_{M}\left(\Gamma_{L J}^{M} \Gamma_{K M}^{I}-\Gamma_{K J}^{M} \Gamma_{L M}^{I}\right) .
$$


It satisfies $R_{J K L}^{I}=-R_{J L K}^{I}$.

If the connection is torsionfree, the curvature tensor satisfies the Bianchi identities

$$
\begin{aligned}
& R(X, Y) Z+R(Z, X) Y+R(Y, Z) X=0, \\
& D_{X} R(Y, Z)+D_{Z} R(X, Y)+D_{Y} R(Z, X)=0 .
\end{aligned}
$$

(If the torsion is not zero, then the Bianchi identities are modified by terms containing the torsion, see [23], volume I page 135.)

It is immediate to see that a for a quasicomplex connection

$$
R_{\bar{\jmath} k \ell}^{i}=0, \quad R_{\bar{\jmath} \bar{k} \bar{\ell}}^{i}=0, \quad R_{j \bar{k} \bar{\ell}}^{i}=0, \quad R_{\bar{\jmath} k \bar{\ell}}^{i}=0 .
$$

From (8) one finds that for a Kähler metric

$$
R_{j k \ell}^{i}=0,
$$

and the only components that can be different from zero are

$$
\begin{aligned}
R_{j k \bar{\ell}}^{i}=-\partial_{\bar{\ell}} \Gamma_{k j}^{i}, & R_{j \bar{k} \ell}^{i}=\partial_{\bar{k}} \Gamma_{j \ell}^{i}, \\
R_{\bar{\jmath} \bar{k} \ell}^{\bar{i}}=-\partial_{\ell} \Gamma_{\bar{k} \bar{\jmath}}^{\bar{\nu}}, & R_{\bar{\jmath} k \bar{\ell}}^{\bar{i}}=\partial_{k} \Gamma_{\bar{\jmath} \ell}^{\bar{\imath}},
\end{aligned}
$$

and those obtained using the symmetry property $R_{J K L}^{I}=-R_{J L K}^{I}$. The upper and lower line are related by complex conjugation.

The Ricci tensor is the contraction $R_{A B}=R_{A C B}^{C}$. We have that

$$
R_{i j}=R_{\bar{\imath} \bar{\jmath}}=0, \quad R_{i \bar{\jmath}}=\bar{R}_{\bar{\imath} j}=-\partial_{\bar{\jmath}} \Gamma_{i k}^{k}=-\partial_{\bar{\jmath}}\left(g^{k \bar{\ell}} \partial_{i} g_{k \bar{\ell}}\right) .
$$

Let $G=\operatorname{det} g_{i \bar{\jmath}}$, then

$$
\partial_{i} G=G g^{k \bar{\ell}} \partial_{i} g_{k \bar{\ell}} \Rightarrow R_{i \bar{\jmath}}=-\partial_{i} \partial_{\bar{\jmath}} \log |G| .
$$

Example 2.5 The complex projective space $\mathbb{C P}^{1}$

We consider the complex projective space of 1-dimensional subspaces in $\mathbb{C}^{2}$. Let $z^{1}, z^{2}$ be the natural coordinate system in $\mathbb{C}^{2}, z^{i}: \mathbb{C}^{2} \rightarrow \mathbb{C}$. They are complex linear maps. Let $U_{1}$ be the set of subspaces $S$ such that $\left.z^{1}\right|_{S} \neq 0$. Then $\left.z^{1}\right|_{S}$ spans the dual space to $S$, so we may write

$$
\left.z^{2}\right|_{S}=\left.t^{1} z^{1}\right|_{S}, \quad t^{1} \in \mathbb{C} .
$$


Each equation as 13 defines a subspace in $U_{1}$, so $t^{1}$ is a complex coordinate in $U_{1}$. In the same way we can define $U_{2}$ as the set of subspaces $S$ such that $\left.z^{2}\right|_{S} \neq 0$. Then we have that

$$
\left.z^{1}\right|_{S}=\left.t^{2} z^{2}\right|_{S}, \quad t^{2} \in \mathbb{C}
$$

and $t^{2}$ is a complex coordinate in $U_{2} \cdot\left\{\left(U_{1}, t^{1}\right),\left(U_{2}, t^{2}\right)\right\}$ is a complex atlas of $\mathbb{C P}^{1}$. In the intersection $U_{1} \cap U_{2}$ the gluing condition is

$$
t^{2}=\frac{1}{t^{1}}
$$

We want to define a Kähler metric on $\mathbb{C P}^{1}$. On $U_{1}$ and $U_{2}$ we consider, respectively, the following real-valued functions:

$$
f_{1}=\left(t^{1} \bar{t}^{1}+1\right), \quad f_{2}=\left(t^{2} \bar{t}^{2}+1\right) .
$$

It is easy to see that the two 2 -forms defined by

$$
\Phi_{1}=4 \mathrm{i} \partial \bar{\partial} \ln f_{1}=-4 \mathrm{i} \frac{1}{f_{1}^{2}} \mathrm{~d} t^{1} \wedge \mathrm{d} \bar{t}^{1}, \quad \Phi_{2}=4 \mathrm{i} \partial \bar{\partial} \ln f_{2}=-4 \mathrm{i} \frac{1}{f_{2}^{2}} \mathrm{~d} t^{2} \wedge \mathrm{d} \vec{t}^{2}
$$

coincide in the intersection, so they define globally a closed 2-form $\Phi$. The Kähler metric is then

$$
g(X, Y)=\Phi(J X, Y)
$$

One can see that it is positive definite by computing it in an open set:

$$
\mathrm{d} s^{2}=\frac{4}{\left(1+t^{1} \bar{t}^{1}\right)^{2}} \mathrm{~d} t^{1} \mathrm{~d} \bar{t}^{1}
$$

\subsection{Hermitian line bundles and fiber metrics}

Hermitian fiber metrics are introduced here and will be used later, in Section 4. The definitions and statements in this section can be found in Refs. [23, 25].

Let $E \rightarrow \mathcal{M}$ be a rank $k$ complex vector bundle over the complex manifold $\mathcal{M}$. Then the fiber at $m \in \mathcal{M}, E_{m}$, is a complex vector space of complex dimension $k$. 
Let us assume that the total space $E$ has a complex structure, that the projection $\pi: E \rightarrow \mathcal{M}$ is a holomorphic map $\}^{4}$ between complex manifolds and that there is a local trivialization $\left\{\mathcal{U}_{A}\right\}_{A \in I}$ such that the maps

$$
\pi^{-1}\left(\mathcal{U}_{A}\right) \rightarrow \mathcal{U}_{A} \times \mathbb{C}^{k}
$$

and their inverses are holomorphic with $\pi^{-1}(m)=E_{m} \approx \mathbb{C}^{k}$. Then we say that $E$ is a holomorphic vector bundle over $\mathcal{M}$.

Example 2.6 The tangent bundle of a complex manifold.

Let $\mathcal{M}$ be a complex manifold. Let $\left(x^{j}, y^{j}\right), j=1, \ldots n$ be coordinates on a neighbourhood $\mathcal{U}$ of $m \in \mathcal{M}$ such that the complex structure on $\mathcal{M}$ is given by

$$
J\left(\frac{\partial}{\partial x^{j}}\right)=\frac{\partial}{\partial y^{j}}, \quad J\left(\frac{\partial}{\partial y^{j}}\right)=-\frac{\partial}{\partial x^{j}}, \quad j=1, \ldots n .
$$

A vector on $m$ is of the form

$$
V_{m}=X_{m}^{j} \frac{\partial}{\partial x^{j}}+Y_{m}^{j} \frac{\partial}{\partial y^{j}} .
$$

The components $X_{m}^{j}, Y_{m}^{j}$ are coordinates on $T_{m}(\mathcal{M})$. On $\pi^{-1}(\mathcal{U})$ we have coordinates $\left(x^{j}, y^{j}, X^{j}, Y^{j}\right)$, and a quasicomplex structure on $T \mathcal{M}$ is given by (14) and

$$
J\left(\frac{\partial}{\partial X^{j}}\right)=\frac{\partial}{\partial Y^{j}}, \quad J\left(\frac{\partial}{\partial Y^{j}}\right)=-\frac{\partial}{\partial X^{j}}, \quad j=1, \ldots n .
$$

The quasicomplex structure is integrable and we have complex coordinates on $\pi^{-1}(\mathcal{U})$ :

$$
\left(z^{j}=x^{j}+\mathrm{i} y^{j}, \quad Z^{j}=X^{j}+\mathrm{i} Y^{j}\right) .
$$

$T \mathcal{M}$ is a complex manifold and a holomorphic vector bundle over $\mathcal{M}$.

A fiber metric on a vector bundle $E \rightarrow \mathcal{M}$ is a smooth assignment of an inner product on each fiber

$$
h_{m}: E_{m} \times E_{m} \longrightarrow \mathbb{R} .
$$

\footnotetext{
${ }^{4}$ That is, a map preserving the complex structures.
} 
If the fiber has a complex structure $J_{m}$ we require that the inner product is Hermitian,

$$
h(J X, J Y)=h(X, Y) \quad \forall X, Y \in E_{m},
$$

and we say that $E$ is a Hermitian vector bundle.

A connection $\nabla$ on $E$ is metric if $\nabla h=0$ (the connection is extended to $\left.E^{*} \otimes E^{*}\right)$. If the bundle is holomorphic, we can ask the covariant derivative of a holomorphic section to be holomorphic,

$$
\nabla s \in \Omega^{1,0}(E) \text { for } s \text { holomorphic. }
$$

There is a unique metric connection satisfying (15); it is the Hermitian connection of the Hermitian vector bundle (see for example [23]).

Let $\left\{s_{a}\right\}_{a=1, \ldots k}$ be a holomorphic frame of the bundle $E$ on a neighbourhood $\mathcal{U}$ of $m \in \mathcal{M}$ (that is, $k$ independent local sections) and $\left\{\alpha^{a}\right\}$ the dual coframe. The connection 1 -form on $\mathcal{U}$, for a connection satisfying $(15)$, is

$$
\left(\nabla s_{b}\right)=\Gamma_{b}^{a} s_{a}=\Gamma_{i}{ }_{b}{ }_{b} \mathrm{~d} z^{i} \otimes s_{a}, \quad i=1, \ldots n, \quad a, b=1, \ldots k,
$$

so the covariant derivative of a holomorphic section $s=a^{a} s_{a}$ is

$$
\nabla s=\left(\partial_{i} a^{a}+\Gamma_{i}{ }_{b}^{a} a^{b}\right) \mathrm{d} z^{i} \otimes s_{a} .
$$

The hermiticity of the fiber metric means

$$
h=2 h_{a \bar{b}} \alpha^{a} \bar{\alpha}^{b}
$$

and the condition for the connection to be metric is

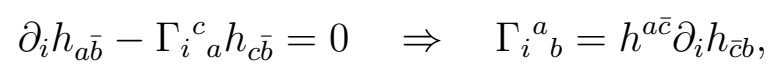

where $h^{a \bar{c}}$ is the inverse matrix of $h_{a \bar{c}}$. The curvature is then

$$
R_{b i \bar{\jmath}}^{a}=-h^{a \bar{c}} \partial_{i} \partial_{\bar{\jmath}} h_{b \bar{c}}+h^{a \bar{c}} h^{d \bar{e}} \partial_{i} h_{b \bar{e}} \partial_{\bar{\jmath}} h_{d \bar{c}} .
$$

We can define the Ricci form of the Hermitian bundle as the trace of the curvature tensor,

$$
\rho=-2 \mathrm{i} R_{a i \bar{\jmath}}^{a} \mathrm{~d} z^{i} \wedge \mathrm{d} \bar{z}^{j} .
$$

If $E$ is a Hermitian line bundle, that is, it has rank 1, then the metric is just

$$
h=\theta(z, \bar{z}) \alpha \bar{\alpha}
$$

and the Ricci form becomes

$$
\rho=-2 \mathrm{i} \bar{\partial} \partial \log |\theta|=-2 \mathrm{i} \partial_{i} \partial_{\bar{\jmath}} \log |\theta| \mathrm{d} z^{i} \wedge \mathrm{d} \bar{z}^{\bar{\jmath}} .
$$




\section{Rigid special Kähler manifolds}

In this section we will deal with rigid special Kähler geometry, or simply special Kähler geometry, as opposed to projective special Kähler geometry, which will be the subject of Section 6 .

\subsection{Some geometric preliminaries}

This part is inspired in the second chapter of Ref. [26].

Let $E$ be a vector bundle over $\mathcal{M}$ with a connection $\nabla$. For every vector field $X$ of $\mathcal{M}$ (section of $T \mathcal{M}$ ), $\nabla$ sends sections of $E$ to sections of $E$,

$$
\nabla_{X}: \Gamma(E) \longrightarrow \Gamma(E)
$$

Let $\Omega_{\mathcal{M}}^{p}(E)=\Lambda^{p}(\mathcal{M}) \otimes \Gamma(E)$ be the space of $E$-valued $p$-forms on $\mathcal{M}$, with $\Omega_{\mathcal{M}}^{0}(E)=\Gamma(E)$. We are going to define the covariant differential $\mathrm{d}_{\nabla}$ : $\Omega_{\mathcal{M}}^{p}(E) \rightarrow \Omega_{\mathcal{M}}^{p+1}(E)$. For 0 -forms we define

$$
\begin{aligned}
\Omega_{\mathcal{M}}^{0}(E) & \stackrel{\mathrm{d}_{\nabla}}{\longrightarrow} \Omega_{\mathcal{M}}^{1}(E) \\
F & \longrightarrow \mathrm{d}_{\nabla} F
\end{aligned} \quad \text { such that } \mathrm{d}_{\nabla} F(X)=\nabla_{X} F, \quad X \in T \mathcal{M}
$$

This definition can be extended to $\Omega_{\mathcal{M}}^{p}(E)$,

$$
\begin{aligned}
\Omega_{\mathcal{M}}^{p}(E) & \stackrel{\mathrm{d}_{\nabla}}{\longrightarrow} \Omega_{\mathcal{M}}^{p+1}(E) \\
F & \longrightarrow \mathrm{d}_{\nabla} F
\end{aligned}
$$

assuming the condition

$$
\mathrm{d}_{\nabla}(\alpha \wedge F)=\mathrm{d} \alpha \wedge F+(-1)^{p} \alpha \wedge \mathrm{d}_{\nabla} F
$$

for $\alpha \in \Lambda^{p}(\mathcal{M})$ and $F \in \Omega_{\mathcal{M}}^{q}(E)$. For example, if $F \in \Omega_{\mathcal{M}}^{1}(E)$, locally $F=\mathrm{d} x^{i} \otimes \alpha_{i}=\mathrm{d} x^{i} \wedge \alpha_{i}$ with $\alpha_{i} \in \Omega_{\mathcal{M}}^{0}(E)$, so

$$
\mathrm{d}_{\nabla} F(X, Y)=-\mathrm{d} x^{i} \wedge \mathrm{d}_{\nabla} \alpha_{i}(X, Y)=-\left(X^{i} \nabla_{Y} \alpha_{i}-Y^{i} \nabla_{X} \alpha_{i}\right) .
$$
then

If $A_{J}^{I}=A_{\mu J}^{I} \mathrm{~d} x^{\mu}$ is the 1 -form connection matrix in an open set $U \subset \mathcal{M}$,

$$
\left(\mathrm{d}_{\nabla} F\right)^{I}=\mathrm{d} F^{I}+A_{J}^{I} \wedge F^{J},
$$


from which it is easy to deduce the standard transformation rule under a local fiber (gauge) transformation

$$
F^{\prime}=U F, \quad\left(\mathrm{~d}_{\nabla} F\right)^{\prime}=U \mathrm{~d}_{\nabla} F, \quad \Rightarrow \quad A^{\prime}=-\mathrm{d} U U^{-1}+U A U^{-1},
$$

where $\left(\mathrm{d}_{\nabla} F\right)^{\prime} \equiv \mathrm{d} F^{\prime}+A^{\prime} F^{\prime}$. Differently than for the ordinary differential, $\mathrm{d}_{\nabla}^{2}$ is not zero in general. In fact,

$$
\left(\mathrm{d}_{\nabla}^{2} F\right)^{I}=\left(\mathrm{d} A_{K}^{I}+A_{J}^{I} \wedge A_{K}^{J}\right) \wedge F^{K}=R_{K}^{I} \wedge F^{K},
$$

where $R$ is the (Lie algebra valued) curvature 2 -form associated to the connection. A flat connection then defines a complex. The de Rham complex is associated to the trivial connection on the trivial bundle $E=\mathcal{M} \times V$.

It is easy to check the Bianchi identity $\mathrm{d}_{\nabla} R=0$. In the associated bundle with typical fiber the Lie algebra, the group acts with the adjoint representation. The covariant differential in such bundle is then

$$
\mathrm{d}_{\nabla} R_{J}^{I}=\mathrm{d} R_{J}^{I}+A_{K}^{I} \wedge R_{J}^{K}-A_{J}^{K} \wedge R_{K}^{I}=0 .
$$

\subsection{Definition of rigid special Kähler manifolds}

Here we follow the first section of Ref. [8].

Definition 3.1 Let $\mathcal{M}$ be a Kähler manifold with Kähler form $\Phi$ and complex structure $J$. A special Kähler structure on $\mathcal{M}$ is a real, flat, torsionfree, symplectic connection $\nabla$ satisfying

$$
\mathrm{d}_{\nabla} J=0 .
$$

$J$ is seen here as a 1-form with values in the tangent bundle $T \mathcal{M}$, and the covariant differential must be interpreted in the sense described in Section 3.1 .

As we have seen, a Kähler manifold is always symplectic, being the Kähler form $\Phi$ its symplectic form. On a symplectic manifold, a linear connection $\nabla$ is said to be symplectic if

$$
\nabla \Phi=0 .
$$

We want to see what is the meaning of the ingredients in this definition. We first examine the implications of the existence of a flat, torsionfree connection. 
Let $U$ be an open set, with coordinates $\left\{x^{I}\right\}_{I=1}^{2 n}$ and a (matrix-valued) connection 1-form $A_{L}^{K}=A_{M L}^{K} \mathrm{~d} x^{M}$.

Due to the flatness condition, $\mathrm{d}_{\nabla}^{2}=0$, so a $\mathrm{d}_{\nabla}$-closed form is locally $\mathrm{d}_{\nabla}$-exact.

Let $\mathbb{1 1}$ be the identity endomorphism in $T \mathcal{M}$. It can be seen as a $T \mathcal{M}$ valued 1-form, $\mathbb{1}=\partial_{I} \otimes d x^{I}$. The torsionfree condition can be expressed as

$$
\mathrm{d}_{\nabla} \mathbb{1}=\left(\mathrm{d}_{\nabla} \mathbb{1}\right)^{K} \partial_{K}=A_{L M}^{K} \mathrm{~d} x^{L} \wedge \mathrm{d} x^{M} \otimes \partial_{K}=0 \Rightarrow A_{L M}^{K}=A_{M L}^{K} .
$$

A local frame on $T \mathcal{M}$ on $U \subset_{\text {open }} \mathcal{M}$ is a set $\left\{\xi_{\alpha}=\xi_{\alpha}^{J} \partial_{J}\right\}_{\alpha=1}^{2 n}$ of $2 n$ local sections of $T U \subset T \mathcal{M}$ that are linearly independent for each point $x \in U$. Since the connection is flat (the curvature tensor is zero), there exists a flat frame, that is,

$$
\nabla_{I} \xi_{\alpha}=0 \quad \text { for } I=1, \ldots 2 n, \quad \text { or equivalently, } \quad \mathrm{d}_{\nabla} \xi_{\alpha}=0
$$

This is because the integrability condition of 21 is

$$
\mathrm{d}_{\nabla}^{2} \xi_{\alpha}=R_{K}^{I} \wedge \xi_{\alpha}^{K} \partial_{I}=0
$$

for the $2 n$ independent sections $\xi_{\alpha}$, which implies necessarily that $R_{K}^{I}=0$.

Let $\theta^{\alpha}=\theta_{I}^{\alpha} \mathrm{d} x^{I}$ be the dual coframe, that is $\theta^{\alpha}\left(\xi_{\beta}\right)=\delta_{\beta}^{\alpha}$. We have that

$$
\theta_{I}^{\alpha} \xi_{\beta}^{I}=\delta_{\alpha}^{\beta}, \quad \Leftrightarrow \quad \xi_{\alpha}^{J} \theta_{I}^{\alpha}=\delta_{I}^{J}
$$

Then we can express

$$
\mathbb{1 1}=\theta^{\alpha} \otimes \xi_{\alpha}=\theta^{\alpha} \wedge \xi_{\alpha}
$$

and

$$
\mathrm{d}_{\nabla} \mathbb{1}=0 \quad \Rightarrow \quad \mathrm{d}_{\nabla} \theta^{\alpha} \wedge \xi_{\alpha}-\theta^{\alpha} \wedge \mathrm{d}_{\nabla} \xi_{\alpha}=0 \quad \Rightarrow \quad \mathrm{d}_{\nabla} \theta^{\alpha}=\mathrm{d} \theta^{\alpha}=0 .
$$

This means that $\theta^{\alpha}=\mathrm{d} t^{\alpha}$ for some functions $t^{\alpha}$. Then $\xi_{\alpha}=\partial / \partial t_{\alpha}$ and $t^{\alpha}$ are local coordinates on $U$ called flat coordinates.

Up to here, we used the fact that the connection is real, flat and torsionfree. We introduce now the additional condition that the connection is symplectic, that is, $\nabla_{I} \Phi=0$. We denote the symplectic matrix as

$$
P=\left(\begin{array}{cc}
0 & \mathbb{1}_{n \times n} \\
-\mathbb{1}_{n \times n} & 0
\end{array}\right) .
$$


The coordinates $t^{\alpha}$ are Darboux coordinates if

$$
\Phi\left(\xi_{\alpha}, \xi_{\beta}\right)=P_{\alpha \beta}, \quad \text { so } \quad \Phi=\frac{1}{2} P_{\alpha \beta} \mathrm{d} t^{\alpha} \wedge \mathrm{d} t^{\beta} .
$$

It is possible to choose the flat coordinates $t^{\alpha}$ in such a way that they are Darboux. This is because

$$
\partial_{I}\left(\Phi\left(\xi_{\alpha}, \xi_{\beta}\right)\right)=\nabla_{I}(\Phi)\left(\xi_{\alpha}, \xi_{\beta}\right)-\Phi\left(\nabla_{I} \xi_{\alpha}, \xi_{\beta}\right)-\Phi\left(\xi_{\alpha}, \nabla_{I} \xi_{\beta}\right)=0,
$$

so $\Phi\left(\xi_{\alpha}, \xi_{\beta}\right)$ is a constant (antisymmetric, non degenerate) matrix which can always be brought to the form (22) by a linear change of coordinates.

We see that the existence of a flat, torsionfree, symplectic connection on $\mathcal{M}$ is equivalent to having a covering by flat Darboux coordinates (it is also said that $\mathcal{M}$ has a flat symplectic structure). If $\left\{q^{\alpha}\right\}_{\alpha=1}^{2 n}$ are also flat Darboux coordinates, we have that the transition functions satisfy

$$
\mathrm{d} q^{\alpha}=\frac{\partial q^{\alpha}}{\partial t^{\beta}} \mathrm{d} t^{\beta}, \quad \nabla_{t^{\gamma}} \mathrm{d} q^{\alpha}=\frac{\partial q^{\alpha}}{\partial t^{\beta} \partial t^{\gamma}} \mathrm{d} t^{\beta}=0
$$

since

$$
\nabla_{t^{\gamma}} \mathrm{d} t^{\beta}=\nabla_{t^{\gamma}} \theta^{\beta}=0
$$

This implies

$$
\frac{\partial q^{\alpha}}{\partial t^{\beta} \partial t^{\gamma}}=0 \Rightarrow q^{\alpha}=A_{\beta}^{\alpha} t^{\beta}+c^{\alpha}
$$

with $A_{\beta}^{\alpha}$ and $c^{\alpha}$ constant. It follows that $A \in \operatorname{Sp}(2 n)$.

Let us now consider the condition (18). In arbitrary coordinates $\left\{x^{I}\right\}_{I=1}^{2 n}$ it becomes

$$
J=J^{I} \partial_{I}=J_{K}^{I} \partial_{I} \otimes \mathrm{d} x^{K}, \quad\left(\mathrm{~d}_{\nabla} J\right)^{I}=\mathrm{d} J^{I}+A_{L}^{I} \wedge J^{L}=0,
$$

which in components reads

$$
\frac{1}{2}\left(\partial_{M} J_{N}^{I}-\partial_{N} J_{M}^{I}\right)+\frac{1}{2}\left(A_{M L}^{I} J_{N}^{L}-A_{N L}^{I} J_{M}^{L}\right)=0 .
$$

(The factor $1 / 2$ appears when $M$ and $N$ are arbitrary, so each strict component is counted twice). This implies, assuming that the connection is torsionfree, that

$$
A_{i \bar{\jmath}}^{I}=A_{\bar{\imath} j}^{I}=0 .
$$


The connection $\nabla$ is a linear connection (a connection on the frame bundle of $\mathcal{M}$ ), so one can compute

$$
\left(\nabla_{I} J\right)_{L}^{K}=\partial_{I} J_{L}^{K}+A_{I M}^{K} J_{L}^{M}-A_{I L}^{M} J_{M}^{K} .
$$

The condition $\nabla_{I} J=0$ together with the torsionfreeness implies, in addition to (24) that

$$
A_{\bar{m} \bar{n}}^{i}=A_{m n}^{\bar{\imath}}=0 \text {. }
$$

If the connection is torsionfree and $\nabla_{I} J=0$ then we have that $\mathrm{d}_{\nabla} J=0$, but the converse is not necessarily true. Then the flat symplectic connection is not necessarily complex.

The complex structure can be written locally in terms of the complex coordinates $\left\{z^{j}\right\}_{j=0}^{n}$ as

$$
J=\mathrm{i}\left(\partial_{z^{j}} \otimes \mathrm{d} z^{j}-\partial_{\bar{z}^{\bar{\jmath}}} \otimes \mathrm{d} \bar{z}^{\bar{\jmath}}\right)=\mathrm{i}\left(\pi^{(1,0)}-\pi^{(0,1)}\right),
$$

where

$$
\pi^{(1,0)}=\partial_{z^{j}} \otimes \mathrm{d} z^{j} \quad \text { and } \quad \pi^{(0,1)}=\partial_{\bar{z}^{\bar{\jmath}}} \otimes \mathrm{d} \bar{z}^{\bar{\jmath}}
$$

are the projectors onto the $T \mathcal{M}^{(1,0)}$ and $T \mathcal{M}^{(0,1)}$ spaces respectively. The condition $\mathrm{d}_{\nabla} J=0$, together with the torsionfreeness, is equivalent to

$$
\mathrm{d}_{\nabla} \pi^{(1,0)}=0
$$

Indeed, one can also write

$$
\mathbb{1}=\pi^{(1,0)}+\pi^{(0,1)}
$$

and the torsionfree condition was expressed as $\mathrm{d}_{\nabla} \mathbb{1 1}=0$.

Using the Poincaré lemma, $\mathrm{d}_{\nabla} \pi^{(1,0)}=0$ implies that locally there exists a complex vector field $\chi$ such that

$$
\nabla \chi=\mathrm{d}_{\nabla} \chi=\pi^{(1,0)},
$$

which is unique up to a flat complex vector field.

Let $\left\{x^{j}, y_{j}\right\}_{j=1}^{n}$ be a flat Darboux coordinate system, that is,

$$
\Phi=\mathrm{d} x^{j} \wedge \mathrm{d} y_{j}, \quad \text { and } \quad \mathrm{d}_{\nabla}\left(\frac{\partial}{\partial x^{j}}\right)=0, \quad \mathrm{~d}_{\nabla}\left(\frac{\partial}{\partial y_{j}}\right)=0
$$


In this coordinate system we denote

$$
\chi=\frac{1}{2}\left(\eta^{i} \frac{\partial}{\partial x^{i}}-\lambda_{j} \frac{\partial}{\partial y_{j}}\right)
$$

where $\eta^{j}, \lambda_{j}$ are complex functions ( $\chi$ is a complex vector field). Taking the covariant differential and using (28) we obtain

$$
\pi^{(1,0)}=\mathrm{d}_{\nabla} \chi=\frac{1}{2}\left(\mathrm{~d} \eta^{j} \otimes \frac{\partial}{\partial x^{j}}-\mathrm{d} \lambda_{j} \otimes \frac{\partial}{\partial y_{j}}\right) .
$$

$\pi^{(1,0)}$ is a $(1,0)$ tensor, so it follows that $\eta^{j}$ and $\lambda_{j}$ are holomorphic functions. Taking the real part of this equation we have, using (27),

$$
\Re\left(\pi^{(1,0)}\right)=\frac{1}{2} \mathbb{1}=\frac{1}{2}\left(\mathrm{~d} x^{j} \otimes \frac{\partial}{\partial x^{j}}+\mathrm{d} y_{j} \otimes \frac{\partial}{\partial y_{j}}\right),
$$

so we can identify

$$
\Re\left(\mathrm{d} \eta^{j}\right)=\mathrm{d} x^{j}, \quad \Re\left(\mathrm{d} \lambda_{j}\right)=-\mathrm{d} y_{j} .
$$

Together with the condition that $\eta^{j}$ and $\lambda_{j}$ are holomorphic, we have that

$$
\mathrm{d} \eta^{j}=\mathrm{d} x^{j}-\mathrm{i} J^{*} \mathrm{~d} x^{j}, \quad \mathrm{~d} \lambda_{j}=-\mathrm{d} y_{j}+\mathrm{i} J^{*} \mathrm{~d} y_{j} .
$$

We want to see under what conditions the sets $\left\{\eta^{j}\right\}$ and $\left\{\lambda_{j}\right\}$ are sets of complex coordinates. Let $\left\{z^{1}, \ldots, z^{n}\right\}$ be complex coordinates with

$$
\begin{aligned}
& z^{l}=\xi^{l}+\mathrm{i} \omega^{l}, \quad \mathrm{~d} z^{l}=\mathrm{d} \xi^{l}+\mathrm{id} \omega^{l}, \quad \frac{\partial}{\partial z^{l}}=\frac{1}{2}\left(\frac{\partial}{\partial \xi^{l}}-\mathrm{i} \frac{\partial}{\partial \omega^{l}}\right), \\
& \frac{\partial}{\partial \xi^{l}}=\frac{\partial}{\partial z^{l}}+\frac{\partial}{\partial \bar{z}^{l}}, \quad \frac{\partial}{\partial \omega^{l}}=\mathrm{i}\left(\frac{\partial}{\partial z^{l}}-\frac{\partial}{\partial \bar{z}^{l}}\right) .
\end{aligned}
$$

We have

$$
\mathrm{d} \eta^{j}=\alpha_{l}^{j}(z) \mathrm{d} z^{l}, \quad \mathrm{~d} \lambda_{j}=\beta_{j l}(z) \mathrm{d} z^{l} .
$$

$\pi^{(1,0)}$ is the projector on the space of holomorphic vectors. This means that it kills all the antiholomorphic vectors and its image is the set of all the holomorphic vectors. Using (30), (34) and (35) we have

$$
\begin{aligned}
& \pi^{(1,0)}\left(\frac{\partial}{\partial \xi^{j}}\right)=\frac{1}{2}\left(\alpha^{l}{ }_{j} \frac{\partial}{\partial x^{l}}-\beta_{l j} \frac{\partial}{\partial y_{l}}\right), \\
& \pi^{(1,0)}\left(\frac{\partial}{\partial \omega^{j}}\right)=\frac{\mathrm{i}}{2}\left(\alpha^{l}{ }_{j} \frac{\partial}{\partial x^{l}}-\beta_{l j} \frac{\partial}{\partial y_{l}}\right) .
\end{aligned}
$$


The set $\left\{\partial / \partial \xi^{l}, \partial / \partial \omega^{l}\right\}$ forms a basis of the tangent space, so the vectors in (36) and (37) are linearly independent. This implies that the $2 n \times n$ matrix

$$
\left(\alpha^{T},-\beta^{T}\right)
$$

has rank $n$ (the superindex $T$ indicates the usual transpose). From the set of $2 n$ functions $\left(\eta^{j}, \lambda_{j}\right)$ we can always select $n$ independent holomorphic functions that form a set of holomorphic coordinates.

From the fact that the symplectic form $\Phi=\mathrm{d} x^{i} \wedge \mathrm{d} y_{j}$ is of type $(1,1)$ and using (32) and (35) in (28), and compare with (4), we obtain as conditions for $\alpha$ and $\beta$

$$
\begin{aligned}
& -\alpha^{T} \beta+\beta^{T} \alpha=0, \\
& \beta^{T} \bar{\alpha}-\alpha^{T} \bar{\beta}=8 \mathrm{i} g,
\end{aligned}
$$

where $g$ is the $n \times n$ matrix $g_{i \bar{\jmath}}$.

These are the equations that we can obtain in general where we have not used any information on the signature of the metric.

\subsection{The signature of the metric}

Let us first assume that the metric is positive definite (Riemannian metric). We want to show that $\alpha$ itself has rank $n$. Suppose that $\operatorname{rank}(\alpha)<n$. Then, there exists a holomorphic vector $c$ such that

$$
\alpha^{j}{ }_{l}(z) c^{l}(z)=0
$$

But then $\beta_{j l} c^{l}(z) \neq 0$, since otherwise the total rank of the matrix 38 would be lower than $n$. This means that there exists a non zero, holomorphic linear combination of the vectors $\left\{\partial / \partial y^{k}\right\}$, namely

$$
\gamma=\sum \tilde{c}_{k}(z) \frac{\partial}{\partial y_{k}} \neq 0, \quad \tilde{c}_{j}(z)=\beta_{j k} c^{k}
$$

Then, as $\gamma$ has only $y$-components,

$$
0=\Phi(\gamma, \bar{\gamma})=g(\gamma, J \bar{\gamma})=g(\gamma,-\mathrm{i} \bar{\gamma})=-\mathrm{i} g(\gamma, \bar{\gamma})
$$

For a Kähler manifold (with positive definite metric)

$$
g(\gamma, \bar{\gamma})=0 \Leftrightarrow \gamma=0
$$


so we have a contradiction and $\alpha$ must have rank $n$. Looking now to 35 we can conclude that $\left\{\eta_{j}\right\}$ is a set of holomorphic coordinates.

In the same way we can prove that $\beta$ has rank $n$ so $\left\{\lambda_{j}\right\}$ is also a set of holomorphic coordinates.

Remark 3.2 Symplectic transformations (Riemannian case).

One can see independently that a real symplectic transformation cannot change the rank of $\alpha$ and $\beta$, provided they satisfy the following conditions:

1. $\alpha$ and $\beta$ have rank $n$.

2. $-\alpha^{T} \beta+\beta^{T} \alpha=0$,

3. $\beta^{T} \bar{\alpha}-\alpha^{T} \bar{\beta}=8 \mathrm{i} g$, where $g$ is the $n \times n$ matrix $g_{i \bar{\jmath}}$.

In our case, conditions 2. and 3. were obtained in (39).

Let us first introduce the vielbein for the metric

$$
\left(\begin{array}{cc}
0 & g \\
g^{T} & 0
\end{array}\right)=E\left(\begin{array}{cc}
0 & \mathbb{1} \\
11 & 0
\end{array}\right) E^{T}, \quad E=\left(\begin{array}{cc}
e & 0 \\
0 & \bar{e}
\end{array}\right), \quad g=e \bar{e}^{T} .
$$

We can define

$$
\alpha=2 \alpha^{\prime} e^{T}, \quad \beta=2 \beta^{\prime} e^{T},
$$

so we can express 2. and 3. as

2. $-\alpha^{\prime T} \beta^{\prime}+\beta^{\prime T} \alpha^{\prime}=0$,

3. $\beta^{\prime T} \bar{\alpha}^{\prime}-\alpha^{\prime T} \bar{\beta}^{\prime}=2 \mathrm{i} 11$.

Let us denote $\alpha^{\prime}=\alpha_{0}^{\prime}+\mathrm{i} \alpha_{1}^{\prime}, \beta^{\prime}=\beta_{0}^{\prime}+\mathrm{i} \beta_{1}^{\prime}$ with $\alpha_{i}^{\prime}$ and $\beta_{i}^{\prime}$ real. We define the matrix

$$
S_{0}=\left(\begin{array}{cc}
\alpha_{0}^{\prime} & \alpha_{1}^{\prime} \\
\beta_{0}^{\prime} & \beta_{1}^{\prime}
\end{array}\right) \text {. }
$$

Then properties 2. and 3. are equivalent to

$$
S_{0}^{T}\left(\begin{array}{cc}
0 & \mathbb{1 1} \\
-\mathbb{1 1} & 0
\end{array}\right) S_{0}=\left(\begin{array}{cc}
0 & \mathbb{1 1} \\
-11 & 0
\end{array}\right) .
$$

(41) means that $S_{0}$ is a symplectic matrix, $S_{0} \in \operatorname{Sp}(2 n, \mathbb{R})$.

We are ready now to prove the statement above. We have that

$$
S_{0}\left(\begin{array}{c}
11 \\
\text { i11 }
\end{array}\right)=\left(\begin{array}{c}
\alpha^{\prime} \\
\beta^{\prime}
\end{array}\right)
$$


We assume that $\operatorname{rank}\left(\alpha^{\prime}\right)=\operatorname{rank}\left(\beta^{\prime}\right)=n$ and that (41) holds. We want to prove that

$$
\text { for } \hat{S}=\left(\begin{array}{cc}
\hat{A} & \hat{B} \\
\hat{C} & \hat{D}
\end{array}\right) \in \operatorname{Sp}(2 n, \mathbb{R}) \text {, the matrix }\left(\begin{array}{c}
\hat{\alpha} \\
\hat{\beta}
\end{array}\right)=\hat{S}\left(\begin{array}{c}
\alpha^{\prime} \\
\beta^{\prime}
\end{array}\right)
$$

is $\operatorname{such}$ that $\operatorname{rank}(\hat{\alpha})=\operatorname{rank}(\hat{\beta})=n$. Let us write

$$
\hat{S}\left(\begin{array}{c}
\alpha^{\prime} \\
\beta^{\prime}
\end{array}\right)=\hat{S} S_{0} S_{0}^{-1}\left(\begin{array}{c}
\alpha^{\prime} \\
\beta^{\prime}
\end{array}\right)=S\left(\begin{array}{c}
11 \\
\text { i11 }
\end{array}\right), \quad \text { with } \quad S=\hat{S} S_{0} .
$$

$S$ is an arbitrary matrix in $\operatorname{Sp}(2 n, \mathbb{R})$, so all we have to prove is that

$$
S\left(\begin{array}{c}
11 \\
\mathrm{i} 11
\end{array}\right)=\left(\begin{array}{ll}
A & B \\
C & D
\end{array}\right)\left(\begin{array}{c}
11 \\
\mathrm{i} 11
\end{array}\right)=\left(\begin{array}{l}
A+\mathrm{i} B \\
C+\mathrm{i} D
\end{array}\right)
$$

is such that $\operatorname{rank}(A+\mathrm{i} B)=\operatorname{rank}(C+\mathrm{i} D)=n$. We consider the matrices

$$
M=\mathrm{i}(A+\mathrm{i} B), \quad N=(C+\mathrm{i} D) .
$$

We have that

$$
\mathcal{A} \equiv M^{\dagger} N=\mathbb{1}-\mathrm{i} \mathcal{A}_{H}, \quad \mathcal{A}_{H}=A^{T} C+B^{T} D,
$$

since $S$ is a symplectic matrix,

$$
A^{T} C=C^{T} A, \quad B^{T} D=D^{T} B, \quad A^{T} D-C^{T} B=\mathbb{1} .
$$

The matrix $\mathcal{A}_{H}$ is therefore also symmetric, and can be diagonalized such that $\mathcal{A}$ is diagonalized with eigenvalues of the form $(1+\mathrm{i} a) \neq 0$. The determinant of $\mathcal{A}$ is the product of its eigenvalues, so it is different from zero. This implies that $\operatorname{det} M \neq 0, \operatorname{det} N \neq 0$, so our statement is proven.

Remark 3.3 Symplectic transformations (pseudo-Riemannian case).

If $g$ has pseudo-Riemannian signature, there are symplectic transformation changing the rank of $\alpha$ and $\beta$ satisfying 1 to 3 in Remark 3.2. It is enough to give one of such symplectic matrices. First we realize that, as before, conditions 2 and 3 can be put as

2. $-\alpha^{\prime T} \beta^{\prime}+\beta^{\prime T} \alpha^{\prime}=0$, 
3. $\beta^{\prime T} \bar{\alpha}^{\prime}-\alpha^{\prime T} \bar{\beta}^{\prime}=2 \mathrm{i} \eta$,

where $\eta$ is the flat pseudo-Riemannian metric. For definiteness, let us assume that the signature of $\eta$ is $(n-1,1)$ (the other cases can be obtained in the same way). We take $\eta$ in the standard form

$$
\eta=\left(\begin{array}{ccccc}
1 & 0 & 0 & 0 & \cdots \\
0 & 1 & 0 & 0 & \cdots \\
\cdots & & & & \\
0 & 0 & \cdots & 0 & 1 \\
0 & 0 & \cdots & 1 & 0
\end{array}\right)
$$

$\eta^{2}=\mathbb{1 1}$ and the vielbein is defined accordingly. We have that the matrix

$$
S_{0}=\left(\begin{array}{cc}
\alpha_{0}^{\prime} & \alpha_{1}^{\prime} \eta \\
\beta_{0}^{\prime} & \beta_{1}^{\prime} \eta
\end{array}\right)
$$

is a symplectic matrix, condition that is equivalent to 2 and 3 . Also, we have that

$$
S_{0}\left(\begin{array}{c}
11 \\
i \eta
\end{array}\right)=\left(\begin{array}{c}
\alpha^{\prime} \\
\beta^{\prime}
\end{array}\right)
$$

so we can bring $\left(\begin{array}{c}\alpha^{\prime} \\ \beta^{\prime}\end{array}\right)$ to the standard form $\left(\begin{array}{c}11 \\ \text { i } \eta\end{array}\right)$ with the symplectic transformation $S_{0}^{-1}$. It is enough to consider the $n=2$ case. The symplectic matrix

$$
S=\left(\begin{array}{cccc}
1 & 0 & 0 & 0 \\
0 & 0 & 0 & -1 \\
0 & 0 & 1 & 0 \\
0 & 1 & 0 & 0
\end{array}\right)
$$

has the property

$$
S\left(\begin{array}{c}
11 \\
\mathrm{i} \eta
\end{array}\right)=S\left(\begin{array}{ll}
1 & 0 \\
0 & 1 \\
0 & \mathrm{i} \\
\mathrm{i} & 0
\end{array}\right)=\left(\begin{array}{cc}
1 & 0 \\
-\mathrm{i} & 0 \\
0 & \mathrm{i} \\
0 & 1
\end{array}\right)=\left(\begin{array}{c}
\alpha^{\prime \prime} \\
\beta^{\prime \prime}
\end{array}\right)
$$

with the property that $\operatorname{det} \alpha^{\prime}=\operatorname{det} \beta^{\prime}=0$, as we wanted to show. The proof in the remark 3.2 is not valid here because the real part of $\mathcal{A}$, determined by $(A+\mathrm{i} B \eta)$ and $(C+\mathrm{i} D \eta)$, would be zero. 
We consider now a pseudo-Riemannian metric (pseudo-Kähler manifold). Notice that in this case (40) is not true since we can have null vectors. In fact, assume that we have a holomorphic vector field $\gamma$ such that $\Phi(\gamma, \bar{\gamma})=0$ and let us consider the vectors

$$
N_{ \pm}=\gamma \pm \bar{\gamma}
$$

Since $g$ is of type $(1,1)$ and $\Phi(\gamma, \bar{\gamma})=-\mathrm{i} g(\gamma, \bar{\gamma})=0$, each one of the terms below is separately 0 ,

$$
g\left(N_{ \pm}, N_{ \pm}\right)=g(\gamma, \gamma)+g(\bar{\gamma}, \bar{\gamma}) \pm 2 g(\gamma, \bar{\gamma})=0
$$

and then $N_{+}$and i $N_{-}$are null, real vectors. On the other hand, if $N$ is a null, real vector, its holomorphic and antiholomorphic extensions $\gamma=N-\mathrm{i} J N$ satisfy $g(\gamma, \bar{\gamma})=0$ and thus $\Phi(\gamma, \bar{\gamma})=0$. We will treat the case of special pseudo-Kähler manifolds in section 3.5 .

We have thus proven in this section that for a positive definite metric, the matrices $\alpha$ and $\beta$ are each of rank $n$. When the metric is not positive definite, this proof breaks down due to null vectors that may be zero modes of these matrices. However, these matrices might even then still be invertible (see the example in Section 3.6). In fact, in Ref. [7] it is proven that with a symplectic rotation we can always bring $\alpha$ to be non degenerate. A sketch of the proof is given in Appendix A.

\subsection{The prepotential}

We come back to the positive definite metric, or, at least that $\alpha$ and $\beta$ are invertible. Then $\left\{\eta^{j}\right\}_{j=1}^{n}$ and $\left\{\lambda_{j}\right\}_{j=1}^{n}$ are called conjugate coordinate systems. Eqs. (26) and (35) then imply

$$
\pi^{(1,0)}=\mathrm{d} \eta^{j} \otimes \frac{\partial}{\partial \eta^{j}}
$$

from which, comparing with (30),

$$
\frac{\partial}{\partial \eta^{j}}=\frac{1}{2}\left(\frac{\partial}{\partial x^{j}}-\tau_{j k} \frac{\partial}{\partial y_{k}}\right), \quad \tau_{j k}=\frac{\partial \lambda_{k}}{\partial \eta^{j}} .
$$


The Kähler form is

$$
\Phi=\mathrm{d} x^{j} \wedge \mathrm{d} y_{j}=-\frac{1}{4}\left(\mathrm{~d} \eta^{j}+\mathrm{d} \bar{\eta}^{\bar{\jmath}}\right) \wedge\left(\tau_{j k} \mathrm{~d} \eta^{k}+\tau_{\bar{\jmath} \bar{k}} \mathrm{~d} \bar{\eta}^{k}\right) .
$$

Since it is of type $(1,1)$, it follows that

$$
\tau_{i j}=\tau_{j i}
$$

so

$$
\Phi=\frac{1}{4} \mathrm{~d} \eta^{i} \wedge \mathrm{d} \bar{\eta}^{\bar{\jmath}}\left(\tau_{i j}-\bar{\tau}_{\bar{\imath} \bar{\jmath}}\right) .
$$

Comparing to (4), we see the metric and the Kähler form become

$$
g_{i \bar{\jmath}}=-\frac{1}{4} \Im\left(\tau_{i j}\right), \quad \Phi=\frac{1}{2} \mathrm{i} \Im\left(\tau_{i j}\right) \mathrm{d} \eta^{i} \wedge \mathrm{d} \bar{\eta}^{\bar{\jmath}} .
$$

Because of (43), there exists a local holomorphic function, determined up to a constant, such that

$$
\lambda_{j}=-8 \frac{\partial \mathcal{F}}{\partial \eta^{j}}, \quad \tau_{i j}=-8 \frac{\partial^{2} \mathcal{F}}{\partial \eta^{i} \partial \eta^{j}} .
$$

$\mathcal{F}$ is called the holomorphic prepotential. In terms of it, the Kähler potential becomes

$$
\mathcal{K}=-\frac{1}{4} \Im\left(\lambda_{k} \bar{\eta}^{k}\right)=2 \Im\left(\frac{\partial \mathcal{F}}{\partial \eta^{k}} \bar{\eta}^{k}\right) .
$$

The coordinate system $\left\{\eta^{j}\right\}_{j=1}^{n}$ is a special coordinate system.

In the particular case in which $\tau_{j k}=\mathrm{i} \delta_{j k}$, then $\eta^{j}=x^{j}+\mathrm{i} y_{j}$, and

$$
\Phi=\frac{\mathrm{i}}{2} \mathrm{~d} \eta^{i} \wedge \mathrm{d} \bar{\eta}^{\bar{\imath}}
$$

so the manifold is locally isometric to $\mathbb{C}^{n}$.

Recovering the flat connection. A structure of special geometry can be given, in an open set, by a holomorphic function $\mathcal{F}(\eta)$ such that $\Im\left(\tau_{i j}\right)$ with $\tau_{i j}$ as in (45), is a non singular, negative definite matrix. The holomorphic coordinates are declared to be special coordinates. From the knowledge of $\mathcal{F}$ we can recover the flat symplectic coordinates

$$
x^{i}=\Re\left(\eta^{i}\right), \quad y_{j}=-\Re\left(\lambda_{j}\right)=8 \Re\left(\frac{\partial \mathcal{F}}{\partial \eta^{j}}\right)
$$


(up to a constant) and also reconstruct the symplectic section

$$
\chi=\frac{1}{2}\left(\eta^{i} \frac{\partial}{\partial x^{i}}-\lambda_{j} \frac{\partial}{\partial y_{j}}\right) .
$$

In the flat coordinates the coefficients of the flat connection are zero (that is, the covariant derivatives are usual derivatives). If we want to use the holomorphic coordinates, these coefficients are not zero anymore. We want to compute them in the coordinates $\left(\eta^{j}\right)$. In order to do this, we perform the coordinate change so the connection transforms as

$$
A^{\prime}=A+\Lambda^{-1} \mathrm{~d} \Lambda
$$

with $A=0$ and

$$
\left(\begin{array}{l}
\mathrm{d} x \\
\mathrm{~d} y
\end{array}\right)=\Lambda\left(\begin{array}{l}
\mathrm{d} \eta \\
\mathrm{d} \bar{\eta}
\end{array}\right)=\frac{1}{2}\left(\begin{array}{cc}
\mathbb{1} & \mathbb{1} \\
-\tau & -\bar{\tau}
\end{array}\right)\left(\begin{array}{l}
\mathrm{d} \eta \\
\mathrm{d} \bar{\eta}
\end{array}\right) .
$$

$A$ and $A^{\prime}$ are considered here as matrices $A_{J}^{I}, \bar{\tau}$ is $\tau_{\bar{\imath} \bar{\jmath}}$ and $\Lambda_{I J}$ is written in terms of blocks of size $n \times n$. We have

$$
\Lambda^{-1}=2\left(\begin{array}{cc}
\beta \bar{\tau} & \beta \\
-\beta \tau & -\beta
\end{array}\right), \quad \beta=(\bar{\tau}-\tau)^{-1}=-\frac{1}{8} \mathrm{i} g^{-1} .
$$

Then

$$
\begin{gathered}
A^{\prime}=\Lambda^{-1} \mathrm{~d} \Lambda=\left(\begin{array}{cc}
-\beta & 0 \\
\beta & 0
\end{array}\right) \mathrm{d} \tau+\left(\begin{array}{cc}
0 & -\beta \\
0 & \beta
\end{array}\right) \mathrm{d} \bar{\tau}, \quad \mathrm{d} \tau_{i j}=\frac{\partial \tau_{i j}}{\partial \eta^{k}} \mathrm{~d} \eta^{k}, \\
\text { with } \quad A^{\prime}=\left(\begin{array}{cc}
A_{k}^{\prime j} & A_{\bar{k}}^{\prime j} \\
A_{k}^{\prime \bar{j}} & A_{\bar{k}}^{\prime \bar{j}}
\end{array}\right) .
\end{gathered}
$$

From this expression one can check that conditions (24) are satisfied.

Let us compute the covariant differential of a vector with only holomorphic components, $H=H^{i} \partial / \partial \eta^{i}$. Notice that, acting on such vector, only the first term in 47) contributes, so

$$
\begin{aligned}
\nabla H & =\left(\nabla_{i} H^{j}\right) \mathrm{d} \eta^{i} \otimes \frac{\partial}{\partial \eta^{j}}+\left(\nabla_{\bar{\imath}} H^{j}\right) \mathrm{d} \bar{\eta}^{\bar{\imath}} \otimes \frac{\partial}{\partial \eta^{j}} \\
& =\partial_{i} H^{j} \mathrm{~d} \eta^{i} \otimes \frac{\partial}{\partial \eta^{j}}+A_{k}^{\prime j} H^{k} \otimes \frac{\partial}{\partial \eta^{j}}+A_{k}^{\prime \bar{j}} H^{k} \otimes \frac{\partial}{\partial \bar{\eta}^{\bar{\jmath}}}+\partial_{\bar{\imath}} H^{j} \mathrm{~d} \bar{\eta}^{\bar{\imath}} \otimes \frac{\partial}{\partial \eta^{j}} \\
& =\partial_{i} H^{j} \mathrm{~d} \eta^{i} \otimes \frac{\partial}{\partial \eta^{j}}+\partial_{\bar{\imath}} H^{j} \mathrm{~d} \bar{\eta}^{\bar{\imath}} \otimes \frac{\partial}{\partial \eta^{j}}-\frac{1}{2} \frac{\partial \tau_{j l}}{\partial \eta^{k}} H^{j} \mathrm{~d} \eta^{k} \otimes \frac{\partial}{\partial y_{l}}
\end{aligned}
$$




$$
\text { since } \frac{\partial}{\partial y_{k}}=2\left(\beta^{j k} \frac{\partial}{\partial \eta^{j}}-\beta^{j k} \frac{\partial}{\partial \bar{\eta}^{j}}\right) \text {. }
$$

Example 3.4 The flat metric on $\mathbb{C}^{n}$

Let $z^{1}, \ldots, z^{n}$ be the standard coordinates in $\mathbb{C}^{n}$. To have a rigid special Kähler structure it is enough to give a holomorphic function $\mathcal{F}\left(z^{1}, \ldots, z^{n}\right)$ such that the matrix

$$
-\Im\left(\frac{\partial^{2} \mathcal{F}}{\partial z^{i} \partial z^{j}}\right)
$$

is positive definite and non degenerate. If we take

$$
\mathcal{F}=\frac{1}{4} \mathrm{i}\left(\left(z^{1}\right)^{2}+\cdots+\left(z^{n}\right)^{2}\right),
$$

we obtain the flat metric on $\mathbb{C}^{n}$

$$
g_{i \bar{\jmath}}=\delta_{i j} .
$$

Example 3.5 The upper half plane

In one complex dimension, we consider the holomorphic prepotential

$$
\mathcal{F}=-\frac{1}{24} \eta^{3},
$$

giving the metric

$$
\mathrm{d} s^{2}=\Im(\eta) \mathrm{d} \eta \mathrm{d} \bar{\eta},
$$

which is positive definite and non degenerate on

$$
\{\eta \in \mathbb{C} / \Im(\eta)>0\} .
$$

From $\mathcal{F}$ we can recover the symplectic coordinates

$$
x=\Re(\eta), \quad y=8 \Re\left(\frac{\partial \mathcal{F}}{\partial \eta}\right)=-\Re\left(\eta^{2}\right) .
$$


denoting $\eta=x+\mathrm{i} p$, we have that $y=p^{2}-x^{2}$ and

$$
\Im(\eta)=p=+\sqrt{y+x^{2}}, \quad y>-x^{2},
$$

from which the metric reads

$$
\mathrm{d} s^{2}=\frac{4\left(y+2 x^{2}\right) \mathrm{d} x^{2}+\mathrm{d} y^{2}+4 x \mathrm{~d} x \mathrm{~d} y}{4 \sqrt{y+x^{2}}} .
$$

This metric is not the Poincaré metric on the upper half plane. From (12) we can see that it has non constant curvature

$$
R=\frac{1}{4(\Im(\eta))^{3}} .
$$

\subsection{The pseudo-Kähler case}

As we have seen at the end of section 3.3 that in the pseudo-Kähler case we cannot conclude the independence of the $\eta^{i}$, so they may not form a complex coordinate system. Nevertheless, the $2 n \times n$ matrix $\left(\alpha^{T},-\beta^{T}\right)$ has still rank $n$, so at each point we can always perform a linear transformation $A$

$$
\left(\begin{array}{l}
\mathrm{d} \eta^{\prime} \\
\mathrm{d} \lambda^{\prime}
\end{array}\right)=A\left(\begin{array}{l}
\mathrm{d} \eta \\
\mathrm{d} \lambda
\end{array}\right)=\left(\begin{array}{c}
\alpha^{\prime} \\
\beta^{\prime}
\end{array}\right)(\mathrm{d} z),
$$

such that the matrix

$$
\left(\begin{array}{l}
\alpha^{\prime} \\
\beta^{\prime}
\end{array}\right)=A\left(\begin{array}{l}
\alpha \\
\beta
\end{array}\right)
$$

has $\alpha^{\prime}$ of rank $n$. Moreover, the linear transformation $A$ can be chosen as a transformation of the symplectic group. A proof of this fact is given in Lemma A1 in Ref. [7]. We reproduce a sketch of the proof and some further comments in Appendix A, Lemma A.1 and Remark A.2.

The conclusion is that there exists a locally finite covering by flat Darboux coordinates such that in each open set the matrix $\alpha$ has rank $n$ and then the functions $\eta_{i}$ are a system of complex coordinates. These will be also called

special coordinates. The calculation of the prepotential in these coordinates follows as in section 3.4 . 
The lesson to learn here is that, unlike the Kähler case, in the pseudoKähler case not all the Darboux coordinates are suitable to construct special complex coordinates, but one can equally cover the manifold with special coordinates. These systems of special Darboux coordinates transform in the intersections between charts with matrices belonging to a subgroup of $\operatorname{Sp}(2 n, \mathbb{R})$, the subgroup that preserves the maximal rank of the block $\alpha$ in the $2 n \times n$ matrix

$$
V=\left(\begin{array}{l}
\alpha \\
\beta
\end{array}\right) .
$$

It is easy to determine this subgroup. First, we notice that the matrices

$$
M_{0}=\left(\begin{array}{cc}
A & 0 \\
C & \left(A^{T}\right)^{-1}
\end{array}\right) \in \operatorname{Sp}(n, \mathbb{R})
$$

form a subgroup, and this subgroup is maximal (we relegate the proof to the Appendix, Lemma A.3). For matrices of this form, we have that $\operatorname{det} A \neq 0$, so the rank of $\alpha$ is preserved. On the other hand, as we proved in Remark 3.3. there exists always a symplectic transformation that does not preserve the rank of $\alpha$. The conclusion is that the flat symplectic structure of the tangent bundle is reduced to the subgroup of matrices

$$
\left\{\left(\begin{array}{cc}
A & 0 \\
C & \left(A^{T}\right)^{-1}
\end{array}\right)\right\} \subset \operatorname{Sp}(n, \mathbb{R}) .
$$

\subsection{A special pseudo-Kähler manifold}

Let $\left(z^{1}, z^{2}\right)$ be holomorphic coordinates on $\mathbb{C}^{2}$ and consider the prepotential

$$
\mathcal{F}=-\frac{1}{8} \mathrm{i} z^{1} z^{2},
$$

then

$$
\begin{aligned}
\tau_{i j} & =-8 \frac{\partial^{2} \mathcal{F}}{\partial z^{i} \partial z^{j}}=\left(\begin{array}{ll}
0 & \mathrm{i} \\
\mathrm{i} & 0
\end{array}\right)=\mathrm{i}\left(\begin{array}{ll}
0 & 1 \\
1 & 0
\end{array}\right), \\
g_{i \bar{\jmath}} & =-\frac{1}{4} \Im\left(\tau_{i j}\right)=-\frac{1}{4}\left(\begin{array}{ll}
0 & 1 \\
1 & 0
\end{array}\right), \\
\mathrm{d} s^{2} & =2 g_{i \bar{\jmath}} \mathrm{d} z^{i} \mathrm{~d} \bar{z}^{j}=-\frac{1}{2} \Im\left(\tau_{i j}\right) \mathrm{d} z^{i} \mathrm{~d} \bar{z}^{j}=-\frac{1}{2}\left(\mathrm{~d} z^{1} \mathrm{~d} \bar{z}^{2}+\mathrm{d} z^{2} \mathrm{~d} \bar{z}^{1}\right),
\end{aligned}
$$


which clearly has signature $(2,2)$ (null vectors always come in pairs, one holomorphic and one antiholomorphic). The Kähler form is

$$
\Phi=-2 \mathrm{i} g_{i \bar{\jmath}} \mathrm{d} z^{i} \wedge \mathrm{d} \bar{z}^{j}=\frac{\mathrm{i}}{2} \Im\left(\tau_{i j}\right) \mathrm{d} z^{i} \wedge \mathrm{d} \bar{z}^{j}=\frac{\mathrm{i}}{2}\left(\mathrm{~d} z^{1} \wedge \mathrm{d} \bar{z}^{2}+\mathrm{d} z^{2} \wedge \mathrm{d} \bar{z}^{1}\right) .
$$

Let us denote

$$
z^{1}=x^{1}+\mathrm{i} y_{2}, \quad z^{2}=x^{2}+\mathrm{i} y_{1},
$$

the real and imaginary parts of the complex holomorphic coordinates. These are the Darboux coordinates of (28). Then the Kähler form takes the standard form

$$
\Phi=\mathrm{d} x^{1} \wedge \mathrm{d} y_{1}+\mathrm{d} x^{2} \wedge \mathrm{d} y_{2},
$$

so $\left(x^{i}, y_{i}\right)$ are symplectic coordinates. For these symplectic coordinates, there is associated a special holomorphic system of coordinates, just as in the Riemannian case.

We want to show now that not all the symplectic coordinate systems have this property when the metric is pseudo-Riemannian. Let us make the following symplectic change of coordinates,

$$
\begin{array}{ll}
x^{\prime 1}=x^{1}, \quad x^{\prime 2}=y_{2}, \quad y_{1}^{\prime}=y_{1}, \quad y_{2}^{\prime}=-x^{2}, \\
\text { with } \quad z^{1}=x^{\prime 1}+\mathrm{i} x^{\prime 2}, \quad z^{2}=-y_{2}^{\prime}+\mathrm{i} y_{1}^{\prime} .
\end{array}
$$

We have

$$
\begin{aligned}
\pi^{(1,0)} & =\mathrm{d} z^{i} \otimes \frac{\partial}{\partial z^{i}}=\mathrm{d} z^{1} \otimes \frac{1}{2}\left(\frac{\partial}{\partial x^{1}}-\mathrm{i} \frac{\partial}{\partial y^{2}}\right)+\mathrm{d} z^{2} \otimes \frac{1}{2}\left(\frac{\partial}{\partial x^{2}}-\mathrm{i} \frac{\partial}{\partial y^{1}}\right) \\
& =\frac{1}{2}\left(\mathrm{~d} z^{1} \otimes \frac{\partial}{\partial x^{1}}+\mathrm{d} z^{2} \otimes \frac{\partial}{\partial x^{2}}-\mathrm{id} z^{2} \otimes \frac{\partial}{\partial y^{1}}-\mathrm{id} z^{1} \frac{\partial}{\partial y^{2}}\right)
\end{aligned}
$$

Comparing this equation with 30

$$
\begin{array}{ll}
\mathrm{d} \eta^{1}=\mathrm{d} z^{1}, & \mathrm{~d} \lambda_{1}=\mathrm{id} z^{2}, \\
\mathrm{~d} \eta^{2}=\mathrm{d} z^{2}, & \mathrm{~d} \lambda_{2}=\mathrm{id} z^{1},
\end{array}
$$

and thus, following 35

$$
\alpha^{T}=\left(\begin{array}{cc}
1 & 0 \\
0 & 1
\end{array}\right), \quad \beta^{T}=\mathrm{i}\left(\begin{array}{cc}
0 & 1 \\
1 & 0
\end{array}\right) .
$$


We can use the new variables $\left(x^{\prime}, y^{\prime}\right)$ defined in (51) to calculate $\left(\eta^{\prime}, \lambda^{\prime}\right)$

$$
\pi^{(1,0)}=\mathrm{d} z^{1} \otimes \frac{1}{2}\left(\frac{\partial}{\partial x^{\prime 1}}-\mathrm{i} \frac{\partial}{\partial x^{\prime 2}}\right)+\mathrm{d} z^{2} \otimes \frac{1}{2}\left(-\frac{\partial}{\partial y_{2}^{\prime}}-\mathrm{i} \frac{\partial}{\partial y_{1}^{\prime}}\right) .
$$

Comparing this equation with 30

$$
\begin{array}{cc}
\mathrm{d} \eta^{\prime}=\mathrm{d} z^{1}, & \mathrm{~d} \lambda^{\prime 1}=\mathrm{id} z^{2}, \\
\mathrm{~d} \eta^{\prime 2}=-\mathrm{id} z^{1}, & \mathrm{~d} \lambda^{\prime 2}=\mathrm{d} z^{2},
\end{array}
$$

then

$$
\alpha^{\prime T}=\left(\begin{array}{cc}
1 & -\mathrm{i} \\
0 & 0
\end{array}\right), \quad \quad \beta^{T}=\left(\begin{array}{cc}
0 & 0 \\
\mathrm{i} & 1
\end{array}\right) .
$$

We compute now the null vector, following the general case explained at the beginning of Section 3.3 . Let $c=\left(c_{1}, c_{2}\right)$ be such that

$$
c \alpha^{\prime T}=\left(c_{1}, c_{2}\right)\left(\begin{array}{cc}
1 & -\mathrm{i} \\
0 & 0
\end{array}\right)=(0,0) \quad \Rightarrow \quad c_{1}=0 .
$$

For any $c=\left(c_{1}, c_{2}\right)$ we have that

$$
c \beta^{T}=\left(c_{1}, c_{2}\right)\left(\begin{array}{cc}
0 & 0 \\
\mathrm{i} & 1
\end{array}\right)=c_{2}(\mathrm{i}, 1)
$$

so $\left(0, c_{2}\right)$ is not a null vector of $\beta^{\prime T}$. The vector $\gamma$ and its complex conjugate $\bar{\gamma}$ are then

$$
\begin{gathered}
\gamma=\mathrm{i} \frac{\partial}{\partial z^{1}}+\frac{\partial}{\partial z^{2}} \\
\bar{\gamma}=-\mathrm{i} \frac{\partial}{\partial \bar{z}^{1}}+\frac{\partial}{\partial \bar{z}^{2}}
\end{gathered}
$$

Then

$$
\Phi(\gamma, \bar{\gamma})=\frac{1}{2} \mathrm{i}\left(\mathrm{d} z^{1} \wedge \mathrm{d} \bar{z}^{2}+\mathrm{d} z^{2} \wedge \mathrm{d} \bar{z}^{1}\right)(\gamma \otimes \bar{\gamma})=0
$$

\subsection{The holomorphic cubic form}

Let $\mathcal{M}$ be a rigid special Kähler manifold. We want to compute the difference between the Levi-Civita connection $D$ and the flat connection $\nabla$. Using the same notation as in Ref. [8], we define the tensor $B_{\mathbb{R}}$ as

$$
B_{\mathbb{R}} \equiv \nabla-D, \quad B_{\mathbb{R}} \in \Omega^{1}\left(\mathcal{M}, \operatorname{End}_{\mathbb{R}} T \mathcal{M}\right) .
$$


Since both connections are symplectic, $D \Phi=0$ and $\nabla \Phi=0$ (see (2) and (19), we have that

$$
\begin{aligned}
\partial_{u}(\Phi(v, w)) & =\nabla_{u}(\Phi(v, w))=\Phi\left(\nabla_{u}(v), w\right)+\Phi\left(v, \nabla_{u}(w)\right), \\
\partial_{u}(\Phi(v, w)) & =D_{u}(\Phi(v, w))=\Phi\left(D_{u}(v), w\right)+\Phi\left(v, D_{u}(w)\right), \\
0 & =\Phi\left(\left(B_{\mathbb{R}}\right)_{u}(v), w\right)+\Phi\left(v,\left(B_{\mathbb{R}}\right)_{u}(w)\right) .
\end{aligned}
$$

This says that the endomorphism $\left(B_{\mathbb{R}}\right)_{u}$, for arbitrary $u$, is in the Lie algebra $\mathfrak{s p}(2 n, \mathbb{R})$ defined by $\Phi$. In components, using (7) and (24), we get

$$
\begin{aligned}
B_{\mathbb{R}}=\nabla-D & =\left(A_{i j}^{k}-\Gamma_{i j}^{k}\right) \mathrm{d} \eta^{i} \otimes \mathrm{d} \eta^{j} \otimes \partial_{k}+A_{i j}^{\bar{k}} \mathrm{~d} \eta^{i} \otimes \mathrm{d} \eta^{j} \otimes \partial_{\bar{k}} \\
& +\left(A_{\bar{\imath} \bar{\jmath}}^{\bar{k}}-\Gamma_{\bar{\imath} \bar{\jmath}}^{\bar{k}}\right) \mathrm{d} \bar{\eta}^{\bar{\imath}} \otimes \mathrm{d} \bar{\eta}^{\bar{\jmath}} \otimes \partial_{\bar{k}}+A_{\bar{\imath} \bar{\jmath}}^{k} \mathrm{~d} \bar{\eta}^{\bar{\imath}} \otimes \mathrm{d} \bar{\eta}^{\bar{\jmath}} \otimes \partial_{k} .
\end{aligned}
$$

Let $u, v, w$ vectors of type $(1,0)$. Then $\left(B_{\mathbb{R}}\right)_{u}(\bar{w})=0$, and the last line of (55), with $w$ replaced by $\bar{w}$, implies that $\Phi\left(\left(B_{\mathbb{R}}\right)_{u}(v), \bar{w}\right)=0$. In components, this means

$$
\left(A_{i j}^{k}-\Gamma_{i j}^{k}\right)=0, \quad\left(A_{\bar{\imath} \bar{\jmath}}^{\bar{k}}-\Gamma_{\bar{\imath} \bar{\jmath}}^{\bar{k}}\right)=0,
$$

where the second one follows by complex conjugation. One can define $B \in$ $\Omega^{1,0}(\operatorname{Hom}(T \mathcal{M}, \overline{T \mathcal{M}}))$ such that

$$
B=A_{i j}^{\bar{k}} \mathrm{~d} \eta^{i} \otimes \mathrm{d} \eta^{j} \otimes \partial_{\bar{k}} \quad \text { so } \quad B_{\mathbb{R}}=B+\bar{B} .
$$

Lowering the antiholomorphic index with the metric, we can define locally a holomorphic 3-tensor,

$$
\Xi_{i j k}=-2 \mathrm{i} g_{i \bar{\ell}} A_{j k}^{\bar{\ell}}, \quad \Leftrightarrow \quad A_{j k}^{\bar{\imath}}=\frac{1}{2} \mathrm{i} g^{\bar{\ell} \ell} \Xi_{\ell j k} .
$$

Using (47) and the fact that $g^{\bar{\jmath} \ell}=8 \mathrm{i} \beta^{\bar{\jmath} \ell}$ we get

$$
\Xi_{i j k}=-\frac{1}{4} \frac{\partial \tau_{i j}}{\partial \eta^{k}}=2 \frac{\partial^{3} \mathcal{F}}{\partial \eta^{i} \partial \eta^{j} \partial \eta^{k}},
$$

from which it follows that $\Xi$ is holomorphic and symmetric.

In Ref. [8] the following global definition is given for this tensor:

$$
\Xi(X, Y, Z)=\Phi\left(\pi^{(1,0)} X,\left(\nabla_{Y} \pi^{(1,0)}\right) Z\right) .
$$


In fact, since $D \pi^{(1,0)}=0$, we can substitute $\nabla$ by $B_{\mathbb{R}}$ in 58 so

$$
\begin{aligned}
\Xi(X, Y, Z) & =\Phi\left(\pi^{(1,0)} X,\left[\left(B_{\mathbb{R}}\right)_{Y}, \pi^{(1,0)}\right] Z\right) \\
& =\Phi\left(\pi^{(1,0)} X,\left[(B+\bar{B})_{Y}, \pi^{(1,0)}\right] Z\right) \\
& =\Phi\left(\pi^{(1,0)} X, B_{Y} \pi^{(1,0)} Z\right),
\end{aligned}
$$

which in components, using (4), gives (56).

It is then clear that given the flat connection $\nabla$ we can determine the cubic form $\Xi$. Conversely, assume that we are given a holomorphic symmetric cubic form $\Xi$ on a Kähler manifold. We can determine a tensor $B_{\mathbb{R}}=B+\bar{B}$ from (56). Then, a new connection is defined by $\nabla=D+B_{\mathbb{R}}$. The symmetry of $\Xi$ guarantees that $\nabla$ is torsionfree, symplectic and satisfies (18), as it follows straightforwardly from (56). The flatness condition imposes some restrictions on $\Xi$. We have to impose $\mathrm{d}_{\nabla}^{2}=0$, with

$$
\mathrm{d}_{\nabla} F=\mathrm{d}_{D} F+B \wedge F+\bar{B} \wedge F
$$

for $F \in \Omega_{\mathcal{M}}^{p}(T \mathcal{M})$. Then, if $R$ is the curvature of the Levi-Civita connection, $\frac{1}{2} R^{I}{ }_{J K L} \mathrm{~d} x^{K} \wedge \mathrm{d} x^{L}$, then

$$
\mathrm{d}_{\nabla}^{2}=0 \quad \Leftrightarrow \quad R+\mathrm{d}_{D} B+\mathrm{d}_{D} \bar{B}+B \wedge \bar{B}+\bar{B} \wedge B=0
$$

Analysing the holomorphic and antiholomorphic components in this equation, we obtain that the following expressions have to cancel separately,

$$
\begin{gathered}
R+B \wedge \bar{B}+\bar{B} \wedge B=0 \\
\mathrm{~d}_{D} B=0 \\
\mathrm{~d}_{D} \bar{B}=0 .
\end{gathered}
$$

Equations (60) and (61) are the complex conjugate of each other. (59) imposes a constraint on the curvature of the Kähler manifold. It should be expressed solely in terms of the holomorphic cubic form. In coordinates this means

$$
R_{i j \bar{k}}^{\ell}=A_{j i}^{\bar{p}} A_{\bar{p} \bar{k}}^{\ell}=\frac{1}{4} g^{\ell \bar{\ell}^{\prime}} g^{p^{\prime} \bar{p}} \Xi_{p^{\prime} j i} \bar{\Xi}_{\bar{p} \bar{\ell}^{\prime} \bar{k}}
$$

(60) imposes a constraint on the metric and the cubic tensor. In components we have

$$
B_{j}^{\bar{k}}=A_{i j}^{\bar{k}} \mathrm{~d} \eta^{i}=-\frac{1}{2 \mathrm{i}} g^{\bar{k} \ell} \Xi_{\ell i j} \mathrm{~d} \eta^{i}
$$


SO

$$
-2 \operatorname{id}_{D} B_{j}^{\bar{k}}=\mathrm{d}_{D} g^{\bar{k} \ell} \wedge \Xi_{\ell i j} \mathrm{~d} \eta^{i}+g^{\bar{k} \ell} \mathrm{d}_{D}\left(\Xi_{\ell i j} \mathrm{~d} \eta^{i}\right)=0 .
$$

Here, $g^{\bar{k} \ell}$ is understood as the components of an element of $\Omega^{0}(\mathcal{M}, T \mathcal{M} \otimes$ $\overline{T \mathcal{M}})$, so its covariant differential is the covariant derivative and it is 0 . Only the last term survives, so we have

$$
\mathrm{d}_{D}\left(\Xi_{\ell i j} \mathrm{~d} \eta^{i}\right)=\left(\partial_{k} \Xi_{l i j}-\Gamma_{k \ell}^{\ell^{\prime}} \Xi_{\ell^{\prime} i j}-\Gamma_{k j}^{j^{\prime}} \Xi_{\ell i j^{\prime}}\right) \mathrm{d} \eta^{k} \wedge \mathrm{d} \eta^{i}=0 .
$$

It is easy to see that for a metric as in (44) and a cubic form as in (58) this equation is satisfied identically. What this argument proves is that (59) and (60), or the equivalent statements $(62)$ and (63), are sufficient conditions to have a flat symplectic connection satisfying the requirements of a rigid special Kähler structure.

Indeed, given a Hermitian metric and and arbitrary holomorphic cubic form with components $\Xi_{i j k}=\Xi_{(i j k)}$, one can construct a torsionfree, symplectic connection as $\nabla=D+B_{\mathbb{R}}$, where the connection coefficients for $B_{\mathbb{R}}$ are determined by (56). This connection, by construction, satisfies $d_{\nabla} J=0$. Then (59), (60) are equivalent to the statement that $\nabla$ is flat, $\left(\mathrm{d}_{\nabla}^{2}=0\right)$. So given a Hermitian metric and a holomorphic cubic form, they will in this case define a special Kähler structure.

\section{Projective Kähler (Kähler-Hodge) manifolds}

\subsection{Affine transformations, isometries and homothetic Killing vectors}

For the results in this section, see ref. [23], Chapter VI.

An affine transformation of a manifold $\mathcal{M}$ with linear connection $\nabla$ is a diffeomorphism $f: \mathcal{M} \rightarrow \mathcal{M}$ whose tangent map Tf $: T \mathcal{M} \rightarrow T \mathcal{M}$ maps any parallel vector field along a curve $\gamma$ into a parallel vector field along the curve $f(\gamma)$. The push-forward by $f$ of a vector field $X$ on $\mathcal{M}$ is

$$
f_{*}(X)=T f \circ X \circ f^{-1},
$$

or, in components,

$$
f_{*} X(x)=\frac{\partial f^{I}}{\partial x^{J}} X^{J}\left(f^{-1}(x)\right) \partial_{I}
$$


If $Y$ and $Z$ are two vector fields on $\mathcal{M}$ and $f$ is an affine transformation, then

$$
\left(f_{*} \nabla_{Y} Z\right)=\nabla_{f_{*} Y}\left(f_{*} Z\right) .
$$

Let $K$ be a vector field on $\mathcal{M}$ and let $\varphi_{t}: U \rightarrow \mathcal{M}$ be the flow of $K$ on a neighbourhood $U$ of $x \in \mathcal{M}, t \in]-\epsilon, \epsilon\left[. \varphi_{t}\right.$ is a local uniparametric group of transformations, and for each $x \in \mathcal{M}, \varphi_{t}(x)$ is an integral curve of $K$ :

$$
\frac{\mathrm{d} \varphi_{t}(x)}{\mathrm{d} t}=K\left(\varphi_{t}(x)\right) .
$$

We say that $K$ is an infinitesimal affine transformation of $\mathcal{M}$ if $\varphi_{t}$ is an affine transformation of $U$ (the connection being the restriction of $\nabla$ to $U$ ). Specifying $f=\varphi_{t}$ in (65) and taking a derivative with respect to $t$ and putting $t=0$ one obtain 5

$$
\mathcal{L}_{K} \circ \nabla_{Y}-\nabla_{Y} \circ \mathcal{L}_{K}=\nabla_{[K, Y]}, \quad \text { for every vector field } Y \text { on } \mathcal{M},
$$

which characterizes $K$ as an infinitesimal affine transformation. (Here $\mathcal{L}_{K}$ stands for the Lie derivative with respect to $K$ ). In components, this condition reads

$$
K^{J} \partial_{J} \nabla_{I} Z^{L}-\nabla_{\sigma}\left(K^{J} \partial_{J} Z^{L}\right)+Z^{J} \nabla_{I} \partial_{J} K^{L}+\left(\partial_{I} K^{J}\right) \nabla_{J} Z^{L}=0 .
$$

The infinitesimal affine transformations form a subalgebra of the Lie algebra of vector fields on $\mathcal{M}$.

For torsionfree connections, 66 reduces to

$$
R(K, Y) Z+\nabla_{Y} \nabla_{Z} K-\nabla_{\nabla_{Y} Z} K=0,
$$

or, in components (as $Z$ is arbitrary)

$$
K^{J} R_{J I K}{ }^{L}+\nabla_{I} \nabla_{K} K^{L}=0,
$$

which was used in Ref. [27] as the definition of symmetry of the physical sigma model, independently of the action (in fact, such action may not exist).

A vector field $X$ on $\mathcal{M}$ is complete if each integral curve $\varphi_{t}(x)$ extends to $t \in]-\infty,+\infty$. This means that the local uniparametric group extends to a global uniparametric group

$$
\begin{aligned}
\mathbb{R} \times \mathcal{M} & \longrightarrow \mathcal{M} \\
(t, x) & \longrightarrow \varphi_{t}(x) .
\end{aligned}
$$

\footnotetext{
${ }^{5}$ One uses here $\left.\frac{\mathrm{d}}{\mathrm{d} t} \varphi_{t *} X\right|_{t=0}=-[K, X]=-\mathcal{L}_{K} X$, where $\left.\varphi_{t}(x)\right|_{t=0}=x$. The first can be derived from (64) with $f=\varphi_{t}$.
} 
We say that $\nabla$ is a complete linear connection if every geodesic extends to $t \in]-\infty,+\infty[$.

The Lie algebra of the Lie group ${ }^{6}$ of affine transformations consists of all infinitesimal affine transformations that are complete. Moreover, if $\nabla$ is complete then all the infinitesimal affine transformations are complete.

Let $\mathcal{M}$ be a manifold with Riemannian metric $g$ and Riemannian connection $\nabla$. An isometry of $\mathcal{M}$ is a transformation that leaves the metric invariant. An isometry is necessarily an affine transformation of $\mathcal{M}$ with respect to the Riemannian connection.

A vector field $X$ is an infinitesimal isometry (or Killing vector) if the uniparametric group of transformations generated by $X$ in a neighbourhood of $x \in \mathcal{M}$ (for arbitrary $x$ ) consists of local isometries. An infinitesimal isometry $X$ is characterized by

$$
\mathcal{L}_{X} g=0,
$$

which implies that the set of infinitesimal isometries is a Lie algebra.

As in the case of affine transformations, the Lie algebra of the Lie group of isometries is the Lie algebra of all complete infinitesimal isometries, and if $\mathcal{M}$ is complete all the infinitesimal isometries are complete.

We say that a transformation $\phi$ of a (pseudo) Riemannian manifold $\mathcal{M}$ is homothetic if there is a positive constant $a^{2}$ (which depends on $\phi$ ) such that

$$
\begin{gathered}
\left(\phi^{*} g\right)_{x}(X, Y)=g_{\phi(x)}(T \phi(X), T \phi(Y))=a^{2} g_{x}(X, Y), \\
\forall X, Y \in T \mathcal{M} \text { and } x \in \mathcal{M} .
\end{gathered}
$$

Notice that the Christoffel symbols for the metrics $g$ and $a^{2} g$ are the same, so the covariant derivatives are the same. It is then easy to see that a homothetic transformation is an affine transformation of the Levi-Civita connection.

An infinitesimal transformation $K$ of a (pseudo) Riemannian manifold is homothetic if its flow is a homothetic transformation in a neighbourhood of each point $x \in \mathcal{M}$. Infinitesimal homothetic transformations are also called homothetic Killing vectors and can be characterized as

$$
\mathcal{L}_{K} g=c g,
$$

\footnotetext{
${ }^{6}$ It is necessary to assume that $\mathcal{M}$ has a finite number of connected components.
} 
for a constant $c$. This can be seen by substituting $\phi=\varphi_{t}$, the flow of $X$, in (70) and taking the derivative with respect to $t$ at $t=0$. We obtain also

$$
c=\left.\frac{\mathrm{d} a^{2}}{\mathrm{~d} t}\right|_{t=0} .
$$

If $D$ is the Levi-Civita connection, then $(71)$ is equivalent to the statement that

$$
g\left(X, D_{Y} K\right)+g\left(Y, D_{X} K\right)=c g(X, Y) \quad \forall X, Y \in T \mathcal{M},
$$

since $D_{K} X-\mathcal{L}_{K} X=D_{X} K$. In components we have

$$
D_{I} K_{J}+D_{J} K_{I}=c g_{I J}
$$

Let us consider the 1 -form $g_{K}(X)=g(K, X)$. If $\nabla$ is a torsionfree connection we have that

$$
\mathrm{d} g_{K}(X, Y)=\nabla_{X}\left(g_{K}\right)(Y)-\nabla_{Y}\left(g_{K}\right)(X) .
$$

This is true for any 1-form. In our case,

$$
\nabla_{X}\left(g_{K}\right)(Y)=\nabla_{X}(g)(K, Y)+g\left(\nabla_{X} K, Y\right) .
$$

If the connection is compatible with the metric, $\nabla_{X}(g)=0$, we have

$$
\mathrm{d} g_{K}(X, Y)=g\left(\nabla_{X} K, Y\right)-g\left(\nabla_{Y} K, X\right),
$$

SO

$$
\mathrm{d} g_{K}=0 \quad \Leftrightarrow \quad g\left(\nabla_{X} K, Y\right)-g\left(\nabla_{Y} K, X\right)=0 \quad \forall X, Y \in T \mathcal{M}
$$

in components

$$
\nabla_{I} K_{J}-\nabla_{J} K_{I}=0
$$

We say that $K$ is a closed homothetic Killing vector if it is a homothetic Killing vector such that $g_{K}$ is a closed 1-form.

If $K$ is a closed, homothetic Killing vector and $D$ is the Levi-Civita connection, then equations 72 and 73 imply that

$$
D_{Y} K=\frac{1}{2} c Y, \quad \forall Y \in T \mathcal{M} .
$$

This condition is also sufficient. In components we have that

$$
D_{I} K^{J}=\frac{1}{2} c \delta_{I}^{J}
$$


Observe that the statement (74) involves only the connection, so we can use it to generalize the concept of closed homothetic Killing vector to any linear connection. For a torsionfree connection $\nabla$, we will say that a vector field is a closed homothetic Killing vector if

$$
\nabla_{Y} K=\frac{1}{2} c Y, \quad \forall Y \in T \mathcal{M}
$$

We would like to see if such a vector is in fact an infinitesimal affine transformation for the linear connection. For an arbitrary torsionfree connection (66) is reduced to (68). Using $(75)$ the last two terms of (68) vanish, so the condition for a closed homothetic Killing vector to be an infinitesimal affine transformation is

$$
R(K, Y) Z=0 .
$$

On the other hand, for an arbitrary connection, the integrability condition of 75$)$ is $R(Y, Z) K=0$ for all $Y$ and $Z$, which implies

$$
R(K, Y) Z-R(K, Z) Y=0
$$

by using the Bianchi identity (9). The symmetric combination in $Y$ and $Z$ is not zero in general.

We conclude that in general for a torsionfree connection, a closed homothetic Killing vector is not necessarily an infinitesimal affine transformation. For a flat, torsionfree connection, $(76)$ is trivial and thus in this case the closed homothetic Killing vector is an infinitesimal affine transformation.

For the Levi-Civita connection (not necessarily flat), we have seen that any homothetic transformation is an affine transformation. In fact, because of the symmetries of the curvature tensor

$$
R(K, Y) Z=0 \Leftrightarrow R(Y, Z) K=0
$$

so $K$ is an infinitesimal affine transformation, even if the connection is not flat.

Let $\mathcal{M}$ be a Kähler manifold with complex structure $J$ and let $g$ be the Hermitian metric. Let $H$ be a holomorphic vector field. The equations above can be extended to the complexified tangent space. We assume that $H$ is a homothetic Killing vector. In components this reads

$$
\mathcal{L}_{H} g_{\alpha \bar{\beta}}=g_{\gamma \bar{\beta}} D_{\alpha} H^{\gamma}=c g_{\alpha \bar{\beta}} \quad \Leftrightarrow \quad D_{Y} H=c Y, \quad Y \text { holomorphic. }
$$


As before, the last expression does not involve explicitly the metric and can be used as a generalization of holomorphic homothetic Killing vector for any linear connection. Since the metric is Hermitian, it is easy to see that $\bar{H}$ is homothetic with the same constant $c$ (real). It follows that $K=\frac{1}{2}(H+\bar{H})$ is also a homothetic Killing vector with constant $c$ while $\hat{K}=-J K=\frac{1}{2 \mathrm{i}}(H-\bar{H})$ is a Killing vector,

$$
\begin{gathered}
\mathcal{L}_{K} g=\frac{1}{2}\left(\mathcal{L}_{H} g+\mathcal{L}_{\bar{H}} g\right)=c g \\
\mathcal{L}_{\hat{K}} g=\frac{1}{2 \mathrm{i}}\left(\mathcal{L}_{H} g-\mathcal{L}_{\bar{H}} g\right)=0 .
\end{gathered}
$$

Notice that $(79)$ can be written in components as

$$
g_{\gamma \bar{\beta}} D_{\alpha} H^{\gamma}-g_{\bar{\gamma} \alpha} D_{\bar{\beta}} H^{\bar{\gamma}}=0,
$$

which is just the requirement that $K$ is closed, so if $H$ is a holomorphic, homothetic Killing vector then $K=\frac{1}{2}(H+\bar{H})$ is a closed homothetic Killing vector.

The converse is also true: if the metric has a closed homothetic Killing vector $K$, then $J K$ is a Killing vector. It also implies the presence of the holomorphic homothetic Killing vector $H=(1-\mathrm{i} J) K$, i.e. satisfying (77).

\section{Example 4.1 Euclidean space.}

We consider $\mathbb{C}^{n}$ with the metric

$$
\mathrm{d} s^{2}=\mathrm{d} z^{\alpha} \mathrm{d} \bar{z}^{\alpha}
$$

We take $H=z^{\beta} \partial / \partial z^{\beta}$. Then $\mathcal{L}_{H} g=g$, so $H$ is an holomorphic, homothetic Killing vector with $c=1$. We define $K=\frac{1}{2}(H+\bar{H})=\frac{1}{2}\left(z^{\beta} \partial / \partial z^{\beta}+\bar{z}^{\beta} \partial / \partial \bar{z}^{\beta}\right)$. Then

$$
g_{K}=\frac{1}{2}\left(z^{\alpha} \mathrm{d} \bar{z}^{\alpha}+\bar{z}^{\alpha} \mathrm{d} z^{\alpha}\right) \quad \Rightarrow \quad \mathrm{d} g_{K}=0 .
$$




\subsection{Definition of projective Kähler manifolds}

Let $\tilde{\mathcal{M}}$ be a complex manifold and let $H$ be a holomorphic vector field. Then $[H, \bar{H}]=0$, so $\{H, \bar{H}\}$ form an integrable distribution on $T \tilde{\mathcal{M}}$. By Frobenius theorem, there is a foliation of $\tilde{\mathcal{M}}$ whose leaves 7 are complex submanifolds of $\tilde{\mathcal{M}}$ whose tangent space is generated by $H$ and $\bar{H}$. If $H \neq 0$ at all points of $\tilde{\mathcal{M}}$ the foliation is regular; then all the leaves have complex dimension 1 . We can define an equivalence relation on $\tilde{\mathcal{M}}$ by declaring as equivalent two points if they belong to the same leaf. Then, if the foliation is regular, the quotient of $\tilde{\mathcal{M}}$ by this relation (the set of all equivalence classes) is a manifold.

Let $K$ and $\hat{K}$ be, as above, the real and imaginary parts of $H$, respectively, so $H=K+\mathrm{i} \hat{K}$. Let $\varphi_{\tau}$ and $\hat{\varphi}_{\sigma}$ the flows of $K$ and $\hat{K}$ respectively,

$$
\frac{\mathrm{d} \varphi_{\tau}(x)}{\mathrm{d} \tau}=K\left(\varphi_{\tau}(x)\right), \quad \frac{\mathrm{d} \hat{\varphi}_{\sigma}(x)}{\mathrm{d} \sigma}=\hat{K}\left(\hat{\varphi}_{\sigma}(x)\right), \quad \varphi_{0}(x)=\hat{\varphi}_{0}(x)=x .
$$

Since $[K, \hat{K}]=0, K$ is invariant under the flow of $\hat{K}$ and viceversa. This in turn implies that

$$
\varphi_{\tau} \circ \hat{\varphi}_{\sigma}=\hat{\varphi}_{\sigma} \circ \varphi_{\tau}
$$

Let us define $\lambda=\tau-\mathrm{i} \sigma$ and $\rho_{\lambda}=\varphi_{\tau} \circ \hat{\varphi}_{\sigma}$; then it is easy to see that

$$
\frac{\mathrm{d} \rho_{\lambda}(x)}{\mathrm{d} \lambda}=\frac{\mathrm{d} \rho_{\lambda}(x)}{\mathrm{d} \tau}+\mathrm{i} \frac{\mathrm{d} \rho_{\lambda}(x)}{\mathrm{d} \sigma}=H\left(\rho_{\lambda}(x)\right), \quad \rho_{0}(x)=x .
$$

$\rho_{\lambda}$ is a local, complex, 1-dimensional group of transformations,

$$
\rho_{\lambda} \circ \rho_{\lambda^{\prime}}=\rho_{\lambda+\lambda^{\prime}}
$$

$H$ is the fundamental vector field of the action of $G$.

We consider now on $\tilde{\mathcal{M}}$ a holomorphic action of $\mathbb{C}^{\times}$.

$$
\begin{aligned}
\mathbb{C}^{\times} \times \mathcal{M} & \longrightarrow \mathcal{M} \\
(b, x) & \longrightarrow \mathfrak{R}_{b}(x),
\end{aligned}
$$

with $b \in \mathbb{C}^{\times}$. Locally, $b=\exp \lambda$ and $\mathfrak{R}_{b}(x)=\rho_{\lambda}(x)$ with $\rho_{\lambda}$ as in 80 . Let $H$ be the (holomorphic) fundamental vector field of this action 80 . The orbits of the action are the integral submanifolds of the foliation defined by $H$. We assume also that the action is free, so the orbits are diffeomorphic to $\mathbb{C}^{\times}$. Since the group is abelian, the left action is also a right action, so $\tilde{\mathcal{M}}$ is a principal $\mathbb{C}^{\times}$-bundle over the orbit space $\tilde{\mathcal{M}} / \mathbb{C}^{\times}$.

\footnotetext{
${ }^{7}$ The leaves of a foliation are disjoint sets whose union is the whole manifold.
} 
Definition 4.2 (Projective Kähler manifold.) Let $\tilde{\mathcal{M}}$ be a (pseudo) Kähler manifold with metric $\tilde{g}$. We assume that on $\tilde{\mathcal{M}}$ there is a free holomorphic action of $\mathbb{C}^{\times}$such that the fundamental vector field $H$ is a non null, holomorphic homothetic Killing vector of the metric $\tilde{g}$ (or the Levi-Civita connection $\tilde{D})$,

$$
\mathcal{L}_{H} \tilde{g}=c \tilde{g} \quad \Leftrightarrow \quad \tilde{D}_{Y} H=c Y \quad \forall Y \in T^{1,0} \tilde{\mathcal{M}},
$$

such that $\tilde{g}(H, \bar{H}) \neq 0$. Then we say that $\mathcal{M}=\tilde{\mathcal{M}} / \mathbb{C}^{\times}$is a (pseudo) projective Kähler manifold.

We are going to show that $\mathcal{M}$ is a Kähler manifold itself, of a particular class. In order to do that, we need to construct a Hermitian line bundle on $\mathcal{M}$. It is in fact inherited from the tangent bundle on $T \tilde{\mathcal{M}}$.

The symplectic and line bundles and the fiber metric. $\tilde{\mathcal{M}}$ has the structure of a principal $\mathbb{C}^{\times}$-bundle over $\mathcal{M}, \pi: \tilde{\mathcal{M}} \rightarrow \mathcal{M}$. As in $((81))$ we denote the finite right action of $\mathbb{C}^{\times}$on $\tilde{\mathcal{M}}$

$$
\begin{aligned}
& \tilde{\mathcal{M}} \longrightarrow \tilde{\mathcal{M}} \\
& \tilde{m} \longrightarrow \mathfrak{R}_{b}(\tilde{m})
\end{aligned} \quad b \in \mathbb{C}^{\times}
$$

with $\Re_{1} \tilde{m}=\tilde{m}$.

Let $T \tilde{\mathcal{M}}$ be the tangent bundle. The tangent of the action above gives an action on $T \tilde{\mathcal{M}}$

$$
\begin{array}{ccc}
T \tilde{\mathcal{M}} & \longrightarrow \tilde{\mathcal{M}} \\
\left(\tilde{m}, v_{\tilde{m}}\right) & \longrightarrow\left(\Re_{b}(\tilde{m}), \mathrm{d} \Re_{b} v_{\tilde{m}}\right) .
\end{array}
$$

$T_{\tilde{m}} \tilde{\mathcal{M}}$ is a complex vector space, so we also have an action of $\mathbb{C}^{\times}$on it. We will simply denote it by multiplication,

$$
\begin{aligned}
T_{\tilde{m}} \tilde{\mathcal{M}} & \longrightarrow T_{\tilde{m}} \tilde{\mathcal{M}} \\
\left(\tilde{m}, v_{\tilde{m}}\right) & \longrightarrow\left(\tilde{m}, b v_{\tilde{m}}\right) .
\end{aligned}
$$

We are going to define an associated bundle to $\tilde{\mathcal{M}}$ using these actions. We identify elements in $T \tilde{\mathcal{M}}$ related by

$$
\left(\tilde{m}, v_{\tilde{m}}\right) \sim\left(\Re_{b}(\tilde{m}), b^{-1} \mathrm{~d} \Re_{b} v_{\tilde{m}}\right) .
$$


It is easy to see that this is an equivalence relation. The quotient space is a complex vector bundle over $\mathcal{M}$ of rank $n+1$, with $\operatorname{dim}_{\mathbb{C}} \mathcal{M}=n$. We will denote it by $\mathcal{H}=T \tilde{\mathcal{M}} / \sim$. It is a bundle over $\mathcal{M}$ associated to the $\mathbb{C}^{\times}$-principal bundle $\tilde{\mathcal{M}} \rightarrow \mathcal{M}[23]$, so there is an action of $\mathbb{C}^{\times}$on it. Also, the underlying real vector bundle of $\mathcal{H}$ (and its complexification) inherit the action of the symplectic group $\operatorname{Sp}(2 n+2, \mathbb{R})$ from $T \tilde{\mathcal{M}}$.

A vector in the kernel of the projection $\operatorname{ker}\left(\left.T \pi\right|_{\tilde{m}}\right) \approx \operatorname{span}_{\mathbb{C}}\{H(\tilde{m})\}$ is a vertical vector. We can consider the subbundle of $T \mathcal{M}$ consisting of vertical vectors. It is a trivial line subbundle of $T \tilde{\mathcal{M}}$, and it projects to a line bundle on $\mathcal{M}$. We will denote it by $L$. Two different trivializations $(\tilde{m}, \lambda H(\tilde{m}))$ and $\left(\tilde{m}^{\prime}, \lambda^{\prime} H\left(\tilde{m}^{\prime}\right)\right)$ (with $\left.\pi\left(\tilde{m}^{\prime}\right)=\pi(\tilde{m})\right)$ are related, according to $(82)$, by

$$
(\tilde{m}, \lambda H(\tilde{m})) \sim\left(\tilde{m}^{\prime}, \lambda^{\prime} H\left(\tilde{m}^{\prime}\right)\right) \quad \Rightarrow \quad \tilde{m}^{\prime}=\mathfrak{R}_{b}(\tilde{m}), \lambda^{\prime}=b^{-1} \lambda,
$$

since 80 implies for the finite global transformation that $\mathrm{d} \Re_{b} H(\tilde{m})=$ $H\left(\Re_{b}(\tilde{m})\right)$. The transition functions of the bundle are of the form $b^{-1}$.

On $L$ we can define a fiber metric. Let $(\tilde{m}, \lambda H(\tilde{m}))$ be a representative of the equivalence class $\left(m, v_{m}\right) \in L$, and the same for $(\tilde{m}, \sigma H(\tilde{m}))$ in $\left(m, u_{m}\right) \in$ $L$. We set

$$
h_{m}\left(v_{m}, \bar{u}_{m}\right)=\tilde{g}_{\tilde{m}}(\lambda H(\tilde{m}), \bar{\sigma} \bar{H}(\tilde{m}))=(\lambda \bar{\sigma}) \tilde{g}_{\tilde{m}}(H(\tilde{m}), \bar{H}(\tilde{m})) .
$$

We remind that $H(\tilde{m})$ is non null by assumption. We just have to check that this definition is independent of the representatives that we have used, so acting with $b \in \mathbb{C}^{\times}$we have

$$
\begin{aligned}
& \tilde{g}_{\tilde{m} b}\left(b^{-1} \lambda H\left(\Re_{b}(\tilde{m})\right), \bar{b}^{-1} \bar{\sigma} \bar{H}\left(\Re_{b}(\tilde{m})\right)\right) \\
& \quad=(\lambda \bar{\sigma})(b \bar{b})^{-1} \tilde{g}_{\mathfrak{R}_{b}(\tilde{m})}\left(H\left(\Re_{b}(\tilde{m})\right), \bar{H}\left(\Re_{b}(\tilde{m})\right)\right) \\
& \quad=(\lambda \bar{\sigma}) \tilde{g}_{\tilde{m}}(H(\tilde{m}), \bar{H}(\tilde{m})),
\end{aligned}
$$

as we wanted to show. The last equality follows from $\left(70\right.$, taking $b \bar{b}=a^{2}$.

We can now define the Kähler structure on $\mathcal{M}$.

The metric and the Kähler potential. Let $\alpha$ be a local basis of $L^{*}$ (a coframe) dual to the frame $\{H\}$ of $L$, so $\alpha(H)=1$. Using the formulae from the end of section 2.3 we have (the index $a$ runs only over one value and can be omitted)

$$
h=\theta \alpha \bar{\alpha}, \quad \theta=2 \tilde{g}_{\tilde{m}}(H(\tilde{m}), \bar{H}(\tilde{m})) .
$$


We want to compute $\theta$ using convenient coordinates in $\tilde{\mathcal{M}}$. Let $z^{i}, i=$ $1, \ldots n$ be complex coordinates on an open set $U \subset \mathcal{M}$. Let $s: U \rightarrow$ $\pi^{-1}(U)$ be a local section on $\mathcal{M}$. Then we can choose the local trivialization $\pi^{-1}(U) \approx U \times \mathbb{C}^{\times}$given by

$$
\tilde{m}=(m, \hat{y} s(m)), \quad m=\pi(\tilde{m}), \hat{y} \in \mathbb{C}^{\times} .
$$

$\left(\hat{z}^{i}, \hat{y}\right)$ are local coordinates on $\pi^{-1}(U) \stackrel{\text { open }}{\subset} \tilde{\mathcal{M}}$. We define homogeneous coordinates $\hat{\eta}^{\alpha}$ on $\pi^{-1}(U)$ as

$$
\hat{\eta}^{0}=\hat{y}, \quad \hat{\eta}^{\alpha}=\hat{y} \hat{z}^{i} \text { for } \alpha=i .
$$

The action of $\mathbb{C}^{\times}$on $\tilde{\mathcal{M}}$ defined in Eq. 81 , expressed

The action of $\mathbb{C}^{\times}$, expressed in these coordinates is simply

$$
\mathfrak{R}_{b}\left(\hat{z}^{i}, y\right)=\left(\hat{z}^{i}, b^{p} \hat{y}\right) \quad \mathfrak{R}_{b}\left(\hat{\eta}^{\alpha}\right)=b^{p} \hat{\eta}^{\alpha}
$$

for an arbitrary $p \in \mathbb{R}$. So the fundamental vector field is

$$
H=\hat{\eta}^{\alpha} \frac{\partial}{\partial \hat{\eta}^{\alpha}}=\hat{y} \frac{\partial}{\partial \hat{y}} .
$$

(One may choose a multiple of it, which by the definition 77 ) amounts to a rescaling of $c$.) The homothety condition is

$$
\mathcal{L}_{H} \tilde{g}_{\alpha \bar{\beta}}=\hat{\eta}^{\gamma} \frac{\partial}{\partial \hat{\eta}^{\gamma}} \tilde{g}_{\alpha \bar{\beta}}+\frac{\partial \hat{\eta}^{\gamma}}{\partial \hat{\eta}^{\alpha}} \tilde{g}_{\gamma \bar{\beta}}=c \tilde{g}_{\alpha \bar{\beta}} .
$$

We make the change of variables

$$
\eta^{\alpha}=\left(\hat{\eta}^{\alpha}\right)^{c}, \quad \Rightarrow \quad H=c \eta^{\alpha} \frac{\partial}{\partial \eta^{\alpha}} .
$$

In these coordinates the homothety condition is

$$
\mathcal{L}_{H} \tilde{g}_{\alpha \bar{\beta}}=c \eta^{\gamma} \frac{\partial}{\partial \eta^{\gamma}} \tilde{g}_{\alpha \bar{\beta}}+c \frac{\partial \eta^{\gamma}}{\partial \eta^{\alpha}} \tilde{g}_{\gamma \bar{\beta}}=c \tilde{g}_{\alpha \bar{\beta}} .
$$

This condition becomes simply

$$
\eta^{\gamma} \frac{\partial}{\partial \eta^{\gamma}} \tilde{g}_{\alpha \bar{\beta}}=0
$$


Together with its complex conjugate, (86) implies the following property of the metric,

$$
\tilde{g}_{\alpha \bar{\beta}}(\lambda \eta, \bar{\lambda} \bar{\eta})=\tilde{g}_{\alpha \bar{\beta}}(\eta, \bar{\eta}) .
$$

If we choose $p=1 / c$, the action $\Re_{b}$ in the coordinates $\eta^{\alpha}$ is

$$
\mathfrak{R}_{b} \eta^{\alpha}=b \eta^{\alpha}
$$

If we denote

$$
\eta^{0}=y, \quad \eta^{i}=y z^{i},
$$

then $z^{i}$ are coordinates on $\mathcal{M}$. The coordinates on $\tilde{\mathcal{M}}$ are also homogeneous coordinates, which we will further use, and from now on $\partial_{\alpha}=\frac{\partial}{\partial \eta^{\alpha}}$. We have that

$$
H=c \eta^{\alpha} \partial_{\alpha}=c y \frac{\partial}{\partial y} \text {. }
$$

The metric in these coordinates can be written in terms of a Kähler potential

$$
\tilde{g}_{\alpha \bar{\beta}}=\partial_{\alpha} \partial_{\bar{\beta}} \mathcal{K} .
$$

The transformation (87) leads to

$$
\mathcal{K}(\eta, \bar{\eta})=\mathcal{K}^{\prime}(\eta, \bar{\eta})+f(\eta)+f^{\prime}(\bar{\eta}),
$$

with

$$
\mathcal{K}^{\prime}(\lambda \eta, \bar{\lambda} \bar{\eta})=(\lambda \bar{\lambda}) \mathcal{K}(\eta, \bar{\eta}) .
$$

Since $\mathcal{K}$ is real, $f=\bar{f}^{\prime}$ and with a Kähler transformation

$$
\mathcal{K} \longrightarrow \mathcal{K}-f-\bar{f}
$$

we can take $\mathcal{K}^{\prime}$ as the Kähler potential. We will denote it as $\mathcal{K}$ from now on, so we have

$$
\mathcal{K}(\lambda \eta, \bar{\lambda} \bar{\eta})=(\lambda \bar{\lambda}) \mathcal{K}(\eta, \bar{\eta}) .
$$

In particular, this implies

$$
\eta^{\gamma} \partial_{\gamma} \mathcal{K}=\mathcal{K}, \quad \bar{\eta}^{\gamma} \partial_{\bar{\gamma}} \mathcal{K}=\mathcal{K}, \quad \eta^{\gamma} \bar{\eta}^{\delta} \partial_{\gamma} \partial_{\bar{\delta}} \mathcal{K}=\mathcal{K} .
$$

so the definition 83 gives

$$
\theta=\tilde{g}_{\alpha \bar{\beta}} H^{\alpha} \bar{H}^{\bar{\beta}}=c^{2} \eta^{\alpha} \bar{\eta}^{\bar{\beta}} \partial_{\alpha} \bar{\partial}_{\bar{\beta}} \mathcal{K}=c^{2} \mathcal{K} .
$$


Let us consider the exact $(1,1)$-form

$$
\tilde{\rho}=2 \tilde{\rho}_{\alpha \bar{\beta}} \mathrm{d} \eta^{\alpha} \wedge \mathrm{d} \bar{\eta}^{\beta}=2\left(-\frac{1}{\mathcal{K}^{2}} \frac{\partial \mathcal{K}}{\partial \eta^{\alpha}} \frac{\partial \mathcal{K}}{\partial \bar{\eta}^{\beta}}+\frac{1}{\mathcal{K}} \frac{\partial^{2} \mathcal{K}}{\partial \eta^{\alpha} \partial \bar{\eta}^{\beta}}\right) \mathrm{d} \eta^{\alpha} \wedge \mathrm{d} \bar{\eta}^{\beta},
$$

and let us denote by i $\rho$ its pull-back by the section $s$,

$$
s^{*} \tilde{\rho}=2 \tilde{\rho}_{\alpha \bar{\beta}} \frac{\partial \eta^{\alpha}}{\partial z^{i}} \frac{\partial \bar{\eta}^{\beta}}{\partial \bar{z}^{\bar{\jmath}}} \mathrm{d} z^{i} \wedge \mathrm{d} \bar{z}^{\bar{\jmath}}=\mathrm{i} \rho .
$$

Using (92), we can see that the result is independent of the section $s$ used. In fact, we have that $\tilde{\rho}=\mathrm{i} \pi^{*} \rho$,

$$
\tilde{\rho}_{\alpha \bar{\beta}}=\mathrm{i} \rho_{i \bar{j}} \frac{\partial z^{i}}{\partial \eta^{\alpha}} \frac{\partial \bar{z}^{\bar{\imath}}}{\partial \bar{\eta}^{\beta}}
$$

where $z^{i}\left(\eta^{\alpha}\right)$ is the expression of the projection map $\pi: \tilde{\mathcal{M}} \rightarrow \mathcal{M}$ in coordinates.

The tensor $\tilde{\rho}_{\alpha \bar{\beta}}$ is degenerate. Indeed, $H^{\alpha}=c \eta^{\alpha}$ is a zero eigenvector due to the identities (93). We want to show that there is no other zero mode, under the assumption that $\tilde{g}_{\alpha \bar{\beta}}$ is non-degenerate. Let us write it as

$$
\tilde{\rho}_{\alpha \bar{\beta}}=\frac{1}{\mathcal{K}} \tilde{g}_{\alpha \bar{\beta}}-\mu_{\alpha} \bar{\mu}_{\beta}=\partial_{\alpha} \partial_{\bar{\beta}} \log |\mathcal{K}|, \quad \mu_{\alpha} \equiv \frac{1}{\mathcal{K}} \partial_{\alpha} \mathcal{K}=\partial_{\alpha} \log |\mathcal{K}|
$$

We assume now that there is a vector $v^{\alpha}$ such that $v^{\alpha} \tilde{\rho}_{\alpha \bar{\beta}}=0$, then we find that

$$
v^{\alpha}=\mathcal{K}\left(v^{\gamma} \mu_{\gamma}\right) \tilde{g}^{\bar{\beta} \alpha} \bar{\mu}_{\beta},
$$

where $\tilde{g}^{\bar{\beta} \alpha}$ is the inverse of $\tilde{g}_{\alpha \bar{\beta}}$. Hence any zero eigenvector is proportional to $\tilde{g}^{\bar{\beta} \alpha} \bar{\mu}_{\beta}$, and thus there is only one zero mode. In particular, we also obtain

$$
H^{\alpha}=\nu \bar{\mu}_{\beta} \tilde{g}^{\bar{\beta} \alpha}
$$

for some undetermined function $\nu(\eta, \bar{\eta})$.

The vectors $\partial_{i}=\eta^{0} \partial / \partial \eta^{i}$ are transversal to $H$, thus the matrix

$$
\rho_{i \bar{\jmath}}=-\mathrm{i} \tilde{\rho}_{\alpha \bar{\beta}} \frac{\partial \eta^{\alpha}}{\partial z^{i}} \frac{\partial \bar{\eta}^{\beta}}{\partial \bar{z}^{\bar{\jmath}}}
$$

is non degenerate. This matrix (or a matrix proportional to it) can therefore be taken to be the metric on $\mathcal{M}$. 
We define therefore the metric on $\mathcal{M}$

$$
g_{i \bar{\jmath}}=\partial_{i} \partial_{\bar{\jmath}}[ \pm \log |\mathcal{K}|]= \pm \mathrm{i} \rho_{i \bar{\jmath}}, \quad \pm=\operatorname{sign} \mathcal{K} .
$$

The reason for the \pm convention will be explained below.

The Ricci form of the Hermitian bundle agrees according to (17) with

$$
\begin{aligned}
\rho & =2 \rho_{i \bar{\jmath}} \mathrm{d} z^{i} \wedge \mathrm{d} \bar{z}^{\bar{\jmath}}=-2 \mathrm{i} \frac{\partial^{2} \log |\mathcal{K}|}{\partial z^{i} \partial \bar{z}^{\bar{\jmath}}} \mathrm{d} z^{i} \wedge \mathrm{d} \bar{z}^{\bar{\jmath}} \\
& =-2 \mathrm{i}\left(-\frac{1}{\mathcal{K}^{2}} \frac{\partial \mathcal{K}}{\partial z^{i}} \frac{\partial \mathcal{K}}{\partial \bar{z}^{\bar{j}}}+\frac{1}{\mathcal{K}} \frac{\partial^{2} \mathcal{K}}{\partial z^{i} \partial \bar{z}^{\bar{\jmath}}}\right) \mathrm{d} z^{i} \wedge \mathrm{d} \bar{z}^{\bar{j}} .
\end{aligned}
$$

We can also compute the signature of the matrix $\tilde{\rho}_{\alpha \bar{\beta}}$. A vector $V=V^{\alpha} \partial_{\alpha}$ is orthogonal to $H$ if $\mu_{\alpha} V^{\alpha}=0$ and the space of such vectors has dimension $n$. For two such vectors, $V$ and $V^{\prime}$ we have

$$
\tilde{g}\left(V, \bar{V}^{\prime}\right)=\mathcal{K} \tilde{\rho}\left(V, \bar{V}^{\prime}\right),
$$

so the signature of $\tilde{\rho}$ in the space orthogonal to $H$ the same than the signature of $\tilde{g}$ in such space up to a sign. Furthermore, the sign in the remaining direction of $\tilde{g}$ is the sign of $\mathcal{K}$ as it follows from (93).

We can choose a section $s$ such that the vectors $\partial_{i}$ have a lift $s_{*} \partial_{i}$ orthogonal to $H$. Then,

$$
\rho_{i \bar{\jmath}}=-\mathrm{i} s^{*} \tilde{\rho}\left(\partial_{i}, \partial_{\bar{\jmath}}\right),
$$

which is actually independent of the section. So the signature of the metric $g$ in $\mathcal{M}$, (97), is the same than the signature of $\tilde{g}$ in the space orthogonal to $H$.

We conclude that $\rho$ defines a symplectic structure compatible with the complex structure, so $\mathcal{M}$ is a Kähler manifold with Kähler metric as in (97). The Kähler form is in the first Chern class of a line bundle. This implies that the Kähler form is integer. Such manifolds are called Kähler-Hodge manifolds in the literature. When $\mathcal{M}$ is compact, this condition implies that $\mathcal{M}$ is a projective variety, so it is embedded in projective space. This is the Kodaira embedding theorem, see for example Ref. [28], page 181.

What we have proven here is that a projective Kähler manifold is a Kähler-Hodge manifold. 


\subsection{The Levi-Civita connection on a Kähler-Hodge manifold}

The previous part leads to consider (97) as the metric on $\mathcal{M}$. One can compute its Levi-Civita connection. However, there is a natural way of inducing a connection on $\mathcal{M}$ from the Levi-Civita connection in $\tilde{\mathcal{M}}$, which gives the same result. It clarifies the geometrical meaning of the metric in the quotient manifold $\mathcal{M}$. We will perform two projections of the connection, first to the bundle $\mathcal{H}=T \tilde{\mathcal{M}} / \sim$ and then to the tangent bundle $T \mathcal{M}$.

Projecting down to the symplectic bundle. Let $X$ be a vector field on $\mathcal{M}$ and $\xi$ a section of $\mathcal{H}$. Let $\pi: \tilde{\mathcal{M}} \rightarrow \mathcal{M}$ and $p: T \tilde{\mathcal{M}} \rightarrow \mathcal{H}$ the natural projections. Let $\tilde{\mathcal{D}}$ denote a linear connection on $T \tilde{\mathcal{M}}$.

The idea is to find adequate lifts $\tilde{X}$ of $X$ and $\tilde{\xi}$ of $\xi$, both vector fields on $\tilde{\mathcal{M}}$, in such way that the covariant derivative $\tilde{\mathcal{D}}_{\tilde{X}} \tilde{\xi}(\tilde{m})$ projects through $p$ to the same vector on $\mathcal{H}$, independently of the point $\tilde{m}$ in the fiber $\pi^{-1}(m)$ where it has been computed. This will define immediately a covariant derivative on $\mathcal{H}$ as

$$
\mathcal{D}_{X} \xi(m)=p\left(\tilde{\mathcal{D}}_{\tilde{X}} \tilde{\xi}(\tilde{m})\right), \quad \pi(\tilde{m})=m .
$$

Let us first define the respective lifts. A local section $\xi$ of $\mathcal{H}$ is specified by associating an equivalence class $\left[\left(\tilde{m}, v_{\tilde{m}}\right)\right]$ to any point $m$, with $\pi(\tilde{m})=m$. We can choose an arbitrary $\tilde{m} \in \pi^{-1}(m)$ and set $\tilde{\xi}(\tilde{m})=v_{\tilde{m}}$. Then $\tilde{\xi}$ is a vector field on $\tilde{\mathcal{M}}$ satisfying (see eq. 82 )

$$
\tilde{\xi}\left(\Re_{b}(\tilde{m})\right)=b^{-1} \mathrm{~d} \Re_{b} \tilde{\xi}(\tilde{m}) \quad \Leftrightarrow \quad \Re_{b *} \tilde{\xi}=b \tilde{\xi} .
$$

There is a one to one correspondence between the set of local sections of $\mathcal{H}$ and the set of local sections of $T \tilde{\mathcal{M}}$ satisfying $(99)$. So $\tilde{\xi}$ is a natural lift of $\xi$.

Notice that 98 means just that $\tilde{\mathcal{D}}_{\tilde{X}} \tilde{\xi}$ is a vector field on $\tilde{\mathcal{M}}$ satisfying (99), so it defines a section of $\mathcal{H}$. For any affine transformation $\mathfrak{R}_{b}$ of the connection $\tilde{\mathcal{D}}$, we have that 65

$$
\mathfrak{R}_{b *}\left(\tilde{\mathcal{D}}_{\tilde{X}} \tilde{\xi}\right)=\tilde{\mathcal{D}}_{\mathfrak{R}_{b *} \tilde{X}}\left(\mathfrak{R}_{b *} \tilde{\xi}\right)
$$

so all we need to complete the definition (98) are lifts satisfying

$$
\begin{gathered}
\Re_{b *} \tilde{\xi}=b \tilde{\xi} \\
\Re_{b *} \tilde{X}=\tilde{X}
\end{gathered}
$$


100 is already guaranteed. There are many lifts of the vector field $X$ to $T \tilde{\mathcal{M}}$, but we have a connection on the principal bundle $\tilde{\mathcal{M}}$ (or on its associated bundle $L$ ), so it is natural to consider the horizontal lift. Horizontal lifts satisfy (101), so this will show the existence of the induced connection on $\mathcal{H}$. Note that for the Levi-Civita connection or for an arbitrary flat connection, $\mathfrak{R}_{b}$ are affine transformations, so the result applies for these cases of special interest.

To understand the horizontal lift we introduce the definition of connection on a principal bundle as a Lie algebra valued 1-form. The relation with the standard covariant derivative in the associated vector bundles can be found in many places, (see for example Ref. [23]). For completeness we give a brief outline in the Appendix B.

Definition 4.3 Let $G$ be a Lie group and $\mathfrak{g}$ its Lie algebra. A connection on a principal $G$-bundle $P \stackrel{\pi}{\rightarrow} \mathcal{M}$ can be given by a $\mathfrak{g}$-valued 1 -form $\omega$ on $P$ such that:

(i). If $A$ is a fundamental vector field, generating the action of $G$ on the fibre, associated to $\hat{A} \in \mathfrak{g}$ then $\omega(A)=\hat{A}$.

(ii). $\mathfrak{R}_{b}{ }^{*} \omega=\operatorname{Ad}_{b^{-1}} \omega=b^{-1} \omega b, b \in G$. (Ad is the adjoint representation of $G)$.

A horizontal vector $X_{u}$ is a vector in $T_{u} P$ satisfying $\omega\left(X_{u}\right)=0$. In fact, $\omega$ defines a distribution of horizontal spaces on $T P$, denoted by $T P^{h}$. At each point $u$ with $\pi(u)=m$, the horizontal space is mapped isomorphically to $T_{m} \mathcal{M}$. If $T P^{v}=\operatorname{ker}\left(\left.T \pi\right|_{u}\right)$ is the set of vertical vectors tangent to the fiber, then $T_{u} P=T_{u} P^{h} \oplus T_{u} P^{v}$. Moreover, (ii) implies that the distribution is equivariant, that is

$$
T_{\Re_{b}(u)} P^{h}=T \Re_{b}\left(T_{u} P^{h}\right) .
$$

Let $X$ be a vector field on $T \mathcal{M}$. One can prove that there is a unique vector field $\tilde{X}$ on $T P$ such that $T \pi(\tilde{X})=X$ and $\tilde{X}(\tilde{m})$ is horizontal for every $\tilde{m}$. It is the horizontal lift of $X$.

The equivariance of horizontal subspaces, (102), implies (101) as we wanted to show. One can also show that any horizontal vector field on $\tilde{\mathcal{M}}$ satisfying the invariance condition (101) is the lift of a vector field on $\mathcal{M}$. 
One can prove that if $\tilde{X}$ and $\tilde{Y}$ are horizontal lifts of $X$ and $Y$ respectively, then $[\tilde{X}, \tilde{Y}]$ is the horizontal lift of $[X, Y]$. So if $\tilde{\mathcal{D}}$ is a flat connection (as $\tilde{\nabla}$ ) then the induced connection $\mathcal{D}$ on $\mathcal{H}$ is also a flat connection.

Example 4.4 Horizontal lift in $\tilde{\mathcal{M}}$.

As an example that we will use in the following, we are going to compute the horizontal lift of a holomorphic vector $X$ on $T \mathcal{M}$ to $T \tilde{\mathcal{M}}$ for the Hermitian connection.

In the coordinates $(89)$ we have

$$
\mathfrak{R}_{b}(m, y)=(m, b y), \quad \mathrm{d} \Re_{b}=\mathrm{d} z^{i} \otimes \partial_{i}+b \mathrm{~d} y \otimes \partial_{y} .
$$

The connection 1-form and its pull back are

$$
\omega=\omega_{y} \mathrm{~d} y+\kappa_{i} \mathrm{~d} z^{i}, \quad s^{*} \omega=\kappa_{i} \mathrm{~d} z^{i} .
$$

$\kappa_{i}$ is determined by the pull back, which from (16) and (94) is

$$
\kappa_{i}=\mathcal{K}^{-1} \partial_{i} \mathcal{K}=\partial_{i} \log |\mathcal{K}| .
$$

The other component, $\omega_{y}$, is determined by conditions (i) and (ii) in Definition 4.3. Since $A=y \partial_{y}$ and $\hat{A}=1$, (i) implies $\omega_{y}=y^{-1}$. (ii) is then satisfied.

The connection 1-form is then

$$
\omega=y^{-1} \mathrm{~d} y+\kappa_{i} \mathrm{~d} z^{i}=y^{-1} \mathrm{~d} y+\partial_{i} \log |\mathcal{K}| \mathrm{d} z^{i} .
$$

A vector on $T \tilde{\mathcal{M}}, v=v^{i} \partial_{i}+v^{y} \partial_{y}$ is horizontal if and only if

$$
y^{-1} v^{y}+\kappa_{i} v^{i}=0 .
$$

If $v$ is arbitrary, then $v=v^{h}+v^{v}$ with

$$
v^{h}=v^{i} \partial_{i}-y \kappa_{i} v^{i} \partial_{y}, \quad v^{v}=\left(v^{y}+y \kappa_{i} v^{i}\right) \partial_{y} .
$$

$v^{h}$ is the horizontal projection of $v$.

A vector $\tilde{X}=\tilde{X}^{y} \partial_{y}+\tilde{X}^{i} \partial_{i}$ is the horizontal lift of $X=X^{i} \partial_{i}$ if

$$
\begin{array}{rll}
T \pi(\tilde{X})=X & \text { i.e. } & \tilde{X}^{i}=X^{i}, \\
\omega(\tilde{X})=0 & \text { i.e. } & \tilde{X}^{y}=-y \kappa_{i} \tilde{X}^{i},
\end{array}
$$

so

$$
\tilde{X}=X^{i} \partial_{i}-y \kappa_{i} X^{i} \partial_{y}
$$


Projecting down to the tangent bundle. Let us consider the subbundle of $\mathcal{H}$ formed by equivalence classes $\left[\left(\tilde{m}, v_{\tilde{m}}\right)\right]$ such that $v_{\tilde{m}}$ is a horizontal vector. Notice that, due to 102$) b^{-1} \mathrm{~d} \Re_{b} v_{\tilde{m}}$ is horizontal if so is $v_{\tilde{m}}$. We will denote this bundle by $\operatorname{hor}(\mathcal{H})$. We have the following lemma:

Lemma $4.5 \operatorname{hor}(\mathcal{H}) \approx L \otimes T \mathcal{M}$.

Proof. Let $\left[\left(\tilde{m}, v_{\tilde{m}}^{h}\right)\right]$ be an element of $\operatorname{hor}(\mathcal{H})$. We can map it to $T_{m} \mathcal{M}$ with the projection $v_{m}=\mathrm{d} \pi v_{\tilde{m}}^{h} \in T_{m} \mathcal{M}$. If we choose another representative of the same equivalence class, $\left(\mathfrak{R}_{b}(\tilde{m}), v_{\mathfrak{R}_{b}(\tilde{m})}^{\prime h}\right)$, with $v_{\mathfrak{R}_{b}(\tilde{m})}^{\prime h}=b^{-1} \mathrm{~d} \mathfrak{R}_{b} v_{\tilde{m}}^{h}$ we obtain another vector on $T_{m} \mathcal{M}, v_{m}^{\prime}=b^{-1} v_{m}$. The natural projection applied to $\operatorname{hor}(\mathcal{H})$ defines then a section of $L \otimes T \mathcal{M}$.

In the other direction, let $X_{m} \in T_{m} \mathcal{M}$ and $\sigma \in L$. We consider the horizontal lift of $\sigma \otimes X_{m}$ to $L \otimes T \tilde{\mathcal{M}}$ for some choice of $\tilde{m} \in \pi^{-1}(m)$ and we denote it by $\sigma \otimes X_{\tilde{m}}^{h}$. Then we consider the equivalence class $[(\tilde{m}, \sigma \otimes$ $\left.\left.X_{\tilde{m}}^{h}\right)\right] \in L \otimes \operatorname{hor}(\mathcal{H})$. Let $\tilde{m}^{\prime}=\mathfrak{R}_{b}(\tilde{m})$ another choice and $\sigma^{\prime}=b \sigma$. Then we have the equivalence class $\left[\left(\tilde{m}^{\prime}, \sigma^{\prime} \otimes X_{\tilde{m}^{\prime}}^{h}\right)\right]$, with $X_{\tilde{m}^{\prime}}^{h}=\mathrm{d} \Re_{b} X_{\tilde{m}}^{h}$. Since $\left(\tilde{m}, X_{\tilde{m}}^{h}\right) \sim\left(\tilde{m}^{\prime}, b^{-1} X_{\tilde{m}^{\prime}}^{h}\right)$, then $\left(\tilde{m}^{\prime}, \sigma^{\prime} \otimes X_{\tilde{m}^{\prime}}^{h}\right) \sim\left(\tilde{m}, \sigma \otimes X_{\tilde{m}}^{h}\right)$, as we wanted to show.

Let $\mathcal{D}$ be a connection on $\mathcal{H}$ and let $p_{h}: \mathcal{H} \rightarrow \operatorname{hor}(\mathcal{H})$ be the natural projection. We can define a connection on $\operatorname{hor}(\mathcal{H})$ as

$$
\hat{\mathcal{D}}_{X} \xi=p_{h}\left(\mathcal{D}_{X} \xi\right), \quad \text { with } X \in T \mathcal{M} \text {, and } \xi \text { a section in } \operatorname{hor}(\mathcal{H}) \subset \mathcal{H} \text {. }
$$

We want to compute $\hat{\mathcal{D}}$ in coordinates. As before, let $s$ be a local section of $\tilde{\mathcal{M}}$, so $\tilde{m}=(m, y s(m)) \in \tilde{\mathcal{M}}$ and let $\left\{z^{i}\right\}$ be local coordinates on $\mathcal{M}$. Then $\left\{y, z^{i}\right\}$ are coordinates on $\tilde{\mathcal{M}}$. We need to compute the horizontal projection of an arbitrary section $\chi$ of $\mathcal{H}, \chi^{h}=p_{h}(\chi)$. The section $\chi$ has a lift $\tilde{\chi}$ to $T \tilde{\mathcal{M}}$ satisfying $(99)$. In coordinates, using the action of $\mathfrak{R}_{b}$ as in 103 , these equations imply the following $y$-dependence:

$$
\tilde{\chi}(y, z)=y^{-1} \chi^{i}(z) \partial_{i}+\chi^{y}(z) \partial_{y}
$$

and according to 105, the horizontal projection is

$$
\tilde{\chi}^{h}(y, z)=\chi^{i}(z)\left(y^{-1} \partial_{i}-\kappa_{i}(z) \partial_{y}\right)
$$

Let $\xi$ be a section of $\operatorname{hor}(\mathcal{H})$, so

$$
\tilde{\xi}=\xi^{i}(z)\left(y^{-1} \partial_{i}-\kappa_{i}(z) \partial_{y}\right)
$$


We have to compute the horizontal projection of $\mathcal{D}_{X} \xi$. Lifting to $\tilde{\mathcal{M}}$, we know that $\left(\tilde{\mathcal{D}}_{\tilde{X}} \tilde{\xi}\right)^{h}$ must be of the same form

$$
\left(\tilde{\mathcal{D}}_{\tilde{X}} \tilde{\xi}\right)^{h}=\chi^{i}\left(y^{-1} \partial_{i}-\kappa_{i}(z) \partial_{y}\right)
$$

for some $\chi^{i}$. So we can identify

$$
\chi^{i}=\left(\hat{\mathcal{D}}_{X} \xi\right)^{i}=X^{j}\left(\partial_{j} \xi^{i}+\hat{\Gamma}_{j k}^{i} \xi^{k}\right)
$$

For vectors of the form 107 and 106

$$
\begin{aligned}
\tilde{\mathcal{D}}_{\tilde{X}} \tilde{\xi}^{j}= & y^{-1} X^{i} \partial_{i} \xi^{j}+y^{-1} \tilde{\Gamma}_{i k}^{j} \xi^{k} X^{i}-\tilde{\Gamma}_{i 0}^{j} X^{i} \kappa_{k} \xi^{k} \\
& +y^{-1} \kappa_{i} X^{i} \xi^{j}-\tilde{\Gamma}_{0 k}^{j} \xi^{k} X^{i} \kappa_{i}+y \kappa_{i} X^{i} \tilde{\Gamma}_{00}^{j} \xi^{k} \kappa_{k} \\
= & y^{-1} \hat{D}_{X} \xi^{j}
\end{aligned}
$$

where we used the coordinates $\left\{y, z^{i}\right\}$ in $\mathcal{M}$ and used the index 0 for the components with respect to $y$. We obtain therefore for the connection coefficients:

$$
\hat{\Gamma}_{i k}^{j}=\tilde{\Gamma}_{i k}^{j}-y \tilde{\Gamma}_{i 0}^{j} \kappa_{k}-y \tilde{\Gamma}_{0 k}^{j} \kappa_{i}+y^{2} \kappa_{i} \kappa_{k} \tilde{\Gamma}_{00}^{j}+\kappa_{i} \delta_{k}^{j} .
$$

The last term is just the connection on $L$, while the rest defines a connection on $T \mathcal{M}$,

$$
\Gamma_{i k}^{j}=\tilde{\Gamma}_{i k}^{j}-y \tilde{\Gamma}_{i 0}^{j} \kappa_{k}-y \tilde{\Gamma}_{0 k}^{j} \kappa_{i}+y^{2} \kappa_{i} \kappa_{k} \tilde{\Gamma}_{00}^{j} .
$$

We have then written $\hat{\mathcal{D}}$ as a connection on $L \otimes T \mathcal{M}$.

We can now compute explicitly the Levi-Civita connection of $\tilde{g}$ in terms of the Levi-Civita connection of $g$, and check that the formula 108 is satisfied in this case. Due to $(92), \mathcal{K}$ is $y \bar{y}$ times a function that depends only on $z$ and $\bar{z}$. The relation between $\tilde{g}_{\alpha \bar{\beta}}$ and $g_{i \bar{\jmath}}$, given by (97), is

$$
\begin{aligned}
& \tilde{g}_{i \bar{\jmath}}=\mathcal{K}\left( \pm g_{i \bar{\jmath}}+\kappa_{i} \bar{\kappa}_{\bar{\jmath}}\right), \quad \tilde{g}_{0 \bar{\imath}}=\frac{\mathcal{K}}{y} \bar{\kappa}_{\bar{\imath}}, \quad \tilde{g}_{0 \overline{0}}=\frac{\mathcal{K}}{y \bar{y}} \\
& \tilde{g}^{\bar{\imath} j}= \pm \frac{1}{\mathcal{K}} g^{\bar{\imath} j}, \quad \tilde{g}^{\bar{\imath} 0}=\mp \frac{y}{\mathcal{K}} g^{\bar{\imath} j} \kappa_{j}, \quad \tilde{g}^{\overline{0} 0}=\frac{y \bar{y}}{\mathcal{K}}\left(1 \pm \kappa_{i} \bar{\kappa}_{\bar{\jmath}} g^{\bar{\jmath} i}\right),
\end{aligned}
$$


where $\kappa_{i}=\partial_{i} \log |\mathcal{K}|$. This leads to the Levi-Civita connection coefficients $\tilde{\Gamma}_{\alpha \beta}^{\gamma}$ :

$$
\begin{aligned}
& \tilde{\Gamma}_{i k}^{j}=g^{j \bar{m}}\left(\frac{1}{\mathcal{K}} \partial_{i} \partial_{\bar{m}} \partial_{k} \mathcal{K}-\frac{1}{\mathcal{K}^{2}} \partial_{\bar{m}} \mathcal{K} \partial_{i} \partial_{k} \mathcal{K}\right)=\Gamma_{i k}^{j}(g)+\kappa_{i} \delta_{k}^{j}+\kappa_{k} \delta_{i}^{j}, \\
& \tilde{\Gamma}_{i 0}^{j}=y^{-1} g^{j \bar{m}} \partial_{\bar{m}} \partial_{i} \log |\mathcal{K}|=y^{-1} \delta_{i}^{j}, \\
& \tilde{\Gamma}_{i j}^{0}=y \Gamma_{i j}^{k}(g) \kappa_{k}+2 y \kappa_{i} \kappa_{j} \pm \frac{y}{\mathcal{K}} \partial_{i} \partial_{j} \mathcal{K}, \\
& \tilde{\Gamma}_{00}^{j}=\tilde{\Gamma}_{0 i}^{0}=\tilde{\Gamma}_{00}^{0}=0,
\end{aligned}
$$

where $\Gamma(g)$ is the Levi-Civita connection of the metric on $\mathcal{M}$ We thus find that indeed $\Gamma_{i k}^{j}$ as determined in $(109)$ are the Christoffel symbols of the Levi-Civita connection on $\mathcal{M}$, as we wanted to show.

\subsection{Examples of Kähler-Hodge manifolds}

Example 4.6 Complex Grassmannian as a Kähler-Hodge manifold.

We consider the Grassmannian manifold of complex $p$-planes in $\mathbb{C}^{p+q}$, denoted by $G(p, q)$. We take

$$
\tilde{\mathcal{M}}=\{Z \mid Z \text { is a }(p+q) \times p \text { matrix of rank } p\} .
$$

We will write

$$
Z=\left(\begin{array}{l}
Z_{0} \\
Z_{1}
\end{array}\right)
$$

with $Z_{0}$ a $p \times p$ matrix and $Z_{1}$ a $q \times p$ matrix. Each $Z$ defines a $p$-plane in $\mathbb{C}^{p+q}$ as the span of the column vectors. Taking linear combinations of these vectors gives the same plane. Then, there is a right action of $\mathrm{GL}(p, \mathbb{C})$ on $\tilde{\mathcal{M}}$ which does not change the $p$-plane. $\tilde{\mathcal{M}} \rightarrow G(p, q)$ is a principal bundle with structure group $\mathrm{GL}(p, \mathbb{C})$.

The group $\operatorname{SL}(p+q)$ acts transitively on $G(p, q)$, but also the action of $\mathrm{SU}(p+q)$ is transitive, with little group $\mathrm{SU}(p) \times \mathrm{SU}(q) \times \mathrm{U}(1)$, so we have that $G(p, q)$ is the Hermitian symmetric space

$$
G(p, q)=\frac{\mathrm{SU}(p+q)}{\mathrm{SU}(p) \times \mathrm{SU}(q) \times \mathrm{U}(1)} .
$$

$G(p, q)$ is a Kähler manifold and we are going to show that it is in fact a Kähler-Hodge manifold. 
An open cover of $G(p, q)$ is given by the open sets with some fixed minor of order $p$ of $Z$ different from zero. Notice that this property is not changed by the right action of $\mathrm{GL}(p, \mathbb{C})$, so it is well defined on the equivalence classes For concreteness, let us fix

$$
U_{0}=\left\{Z=\left(\begin{array}{c}
Z_{0} \\
Z_{1}
\end{array}\right) \in G(p, q) \mid \operatorname{det} Z_{0} \neq 0\right\} .
$$

A $p$-plane in $U_{0}$ can be characterized by a $q \times p$ matrix $T$ such that a vector $\left(z_{1}, \ldots z_{p}, z_{p+1}, \ldots, z_{p+q}\right)$ satisfies

$$
\left(\begin{array}{c}
z_{p+1} \\
\vdots \\
z_{p+q}
\end{array}\right)=T\left(\begin{array}{c}
z_{1} \\
\vdots \\
z_{p}
\end{array}\right) .
$$

In fact, a matrix $Z$ with $\operatorname{det} Z_{0} \neq 0$ is a collection of $p$ column vectors satisfying the above property, so

$$
Z_{1}=T Z_{0} \quad \Leftrightarrow \quad T=Z_{1} Z_{0}^{-1} .
$$

An arbitrary matrix $T$ defines a $p$-plane in $U_{0}$, so we have

$$
U_{0} \approx \mathcal{M}_{q \times p}(\mathbb{C}) \approx \mathbb{C}^{p q},
$$

and the entries of $T$ are local coordinates on $U_{0}$.

The tautological bundle $H \rightarrow G(p, q)$, is the vector bundle with the fiber at each point of $G(p, q)$ the plane that it represents. It is a rank $p$ subbundle of the trivial bundle $G(p, q) \times \mathbb{C}^{p+q}$. It is a bundle associated to the principal bundle $\tilde{\mathcal{M}}$.

On the trivial bundle there is a fiber metric

$$
\left\langle\zeta, \zeta^{\prime}\right\rangle=\zeta^{1} \bar{\zeta}^{11}+\cdots+\zeta^{p+q} \bar{\zeta}^{\prime p+q}
$$

for $\zeta, \zeta^{\prime}$ vectors at a point in $G(p, q)$. It induces a fiber metric on the tautological bundle. A local section on $H$ is given by functions $\zeta^{1}, \ldots \zeta^{p}$, so that $T$ determines the plane:

$$
\zeta(T)=\left(\begin{array}{c}
\zeta^{1} \\
\vdots \\
\zeta^{p} \\
\zeta^{p+1} \\
\vdots \\
\zeta^{p+q}
\end{array}\right)=\left(\begin{array}{l}
11 \\
T
\end{array}\right)\left(\begin{array}{c}
\zeta^{1} \\
\vdots \\
\zeta^{p}
\end{array}\right) .
$$


The Hermitian inner product on the fiber is

$$
\begin{aligned}
\left\langle\zeta(T), \zeta^{\prime}(T)\right\rangle & =\left(\bar{\zeta}^{1}, \ldots, \bar{\zeta}^{p}\right)\left(\mathbb{1}, T^{\dagger}\right)\left(\begin{array}{c}
11 \\
T
\end{array}\right)\left(\begin{array}{c}
\zeta^{1} \\
\vdots \\
\zeta^{p}
\end{array}\right) \\
& =\left(\bar{\zeta}^{\prime 1}, \ldots, \bar{\zeta}^{p}\right)\left(\mathbb{1}+T^{\dagger} T\right)\left(\begin{array}{c}
\zeta_{1} \\
\vdots \\
\zeta_{p}
\end{array}\right)
\end{aligned}
$$

If $\alpha, \beta=1, \ldots p$ then we have the fiber metric

$$
\left\langle\zeta(T), \zeta^{\prime}(T)\right\rangle=h_{\alpha \bar{\beta}} \zeta^{\alpha} \bar{\zeta}^{\prime \beta}, \quad h^{\mathrm{t}}=\mathbb{1}+T^{\dagger} T .
$$

We can write the Hermitian fiber metric as

$$
h=h_{\alpha \bar{\beta}} \mathrm{d} z^{\alpha} \mathrm{d} \bar{z}^{\beta} .
$$

We consider now the line bundle $\Lambda^{p}(H)$ with fiber at a point $x \in G(p, q)$ $\Lambda^{p} H_{x} \approx \Lambda^{p} \mathbb{C}^{p} \approx \mathbb{C}$, i.e. the determinant. The structure group is

$$
\operatorname{GL}(p, \mathbb{C}) / \mathrm{SL}(p, \mathbb{C}) \approx \mathbb{C}^{\times}
$$

Let $\left\{s_{\alpha}(T)\right\}$ be a local frame on $H$, with $h_{\alpha \bar{\beta}}=\left\langle s_{\alpha}, s_{\beta}\right\rangle$. (To compare with (113) it is enough to take $s_{\alpha}(T)=\zeta(T)$ as in (112) with $\zeta^{\alpha}=1$ and the rest $0)$. Then a local section on $\Lambda^{p}(H)$ is of the form

$$
U(T)=u(T) s_{1} \wedge \cdots \wedge s_{p}
$$

There is an induced fiber metric on this bundle given by

$$
H=\operatorname{det}\left(h_{\alpha \bar{\beta}}\right) \mathrm{d} u \mathrm{~d} \bar{u} .
$$

As in (17) we get for the Ricci form associated to the Hermitian connection on the line bundle

$$
\rho_{i \bar{\jmath}}=-\mathrm{i} \partial_{\bar{\jmath}} \partial_{i} \log \operatorname{det}\left(h_{\alpha \bar{\beta}}\right)=-\mathrm{i} \partial_{\bar{\jmath}} \partial_{i} \log \operatorname{det}\left(\mathbb{1}+T^{\dagger} T\right),
$$

where $i, j=1, \ldots p q$ run over all the entries of the matrix $T$. 
Example 4.7 Non compact "Grassmannian" as a Kähler-Hodge manifold.

In the example above, let us change the fiber metric (111) to a pseudoEuclidean one with signature $(p, q)$,

$$
\left\langle\zeta, \zeta^{\prime}\right\rangle=\zeta^{1} \bar{\zeta}^{\prime 1}+\cdots+\zeta^{p} \bar{\zeta}^{\prime p}-\zeta^{p+1} \bar{\zeta}^{\prime p+1}-\cdots-\zeta^{p+q} \bar{\zeta}^{\prime p+q} .
$$

Then, instead of (113) we have

$$
h^{\mathrm{t}}=11-T^{\dagger} T,
$$

so on the points where the matrix $11-T^{\dagger} T$ is positive definite we have a positive definite, non degenerate fiber metric. The space of matrices satisfying this property is a domain in $\mathbb{C}^{p q}$. It is the Hermitian symmetric space

$$
D(p, q)=\frac{\mathrm{SU}(p, q)}{\mathrm{SU}(p) \times \mathrm{SU}(q) \times \mathrm{U}(1)} .
$$

The corresponding expression for the Ricci curvature is proportional, as before, to the standard Kähler metric on this symmetric space.

\section{Conformal calculus}

The ideas described in the previous section originate in physics as a property of certain sigma models of scalar fields coupled to gravity with a scaling symmetry. It is in fact a simplification of what occurs in supergravity (see for example Refs. [29, 30]), but the essential idea can be grasped in this simplification. We first consider the version with real scalars and then we move to Kähler manifolds.

\subsection{Real manifold.}

We consider a nonlinear sigma model of $n$ real scalar fields $\phi^{I}$ with lagrangian

$$
\mathcal{L}_{\mathbb{R}, 0}=-\frac{1}{2} \sqrt{g} g^{\mu \nu} G_{I J} \partial_{\mu} \phi^{I} \partial_{\nu} \phi^{J} .
$$

Here $g_{\mu \nu}$ is the metric of space time, gravitational field, $g^{\mu \nu}$ its inverse, and $g=\left|\operatorname{det} g_{\mu \nu}\right|$. The target space is a real Riemannian manifold with coordinates $\phi^{I}$ and $G_{I J}(\phi)$ is the Riemannian metric. 
We will be interested in the case that the Lagrangian has a dilatation symmetry given at the infinitesimal level by a vector $K=K^{I} \partial_{I}$. Let $D$ be the dimension of space-time. We assume that the vector $K$ is a homothetic Killing vector of the metric $G_{I J}$,

$$
\mathcal{L}_{K} G_{I J}=K^{L} \partial_{L} G_{I J}+\partial_{I} K^{L} G_{L J}+\partial_{J} K^{L} G_{I L}=c G_{I J}
$$

We fix

$$
c=D-2 \text {. }
$$

Then, the Lagrangian $\mathcal{L}_{\mathbb{R}, 0}$ is invariant under the infinitesimal transformations

$$
\begin{aligned}
\delta_{\epsilon} \phi^{I} & =\epsilon K^{I}, \\
\delta_{\epsilon} g_{\mu \nu} & =-2 \epsilon g_{\mu \nu},
\end{aligned}
$$

for an infinitesimal parameter $\epsilon$ independent of de point $x$ in spacetime.

A simple example is when the metric on the target space is such that

$$
G_{I J}(\lambda \phi)=\lambda^{D-4} G_{I J}(\phi)
$$

which means that the vector $K=\phi^{I} \partial_{I}$ is a homothetic Killing vector 71 ) with $c=D-2$. Then the lagrangian is invariant under the set of transformations

$$
\begin{aligned}
\phi^{I} & \longmapsto \lambda \phi^{I}, \\
g_{\mu \nu} & \longmapsto \lambda^{-2} g_{\mu \nu} \quad \lambda \in \mathbb{R},
\end{aligned}
$$

for a constant parameter $\lambda$ (independent of $x$ ). We obtain the infinitesimal transformations 116 by writing $\lambda=1+\epsilon+\mathcal{O}\left(\epsilon^{2}\right)$.

Let us now consider transformations of the form $(116)$ but with a parameter $\epsilon(x)$ depending on the point. Then

$$
\delta_{\epsilon} \mathcal{L}=-\sqrt{g} G_{I J}\left(\partial_{\mu} \epsilon\right) K^{I} \partial_{\nu} \phi^{J} g^{\mu \nu} .
$$

The lagrangian $\mathcal{L}_{\mathbb{R}, 0}$ is not invariant, but this can be remedied if we add an Einstein-Hilbert like term for the spacetime metric

$$
\mathcal{L}_{\mathbb{R}}=-\frac{1}{2} \sqrt{g} G_{I J} \partial_{\mu} \phi^{I} \partial_{\nu} \phi^{J} g^{\mu \nu}-\frac{1}{2} a\left(G_{I J} K^{I} K^{J}\right) \sqrt{g} R(g),
$$


where $R(g)$ is the spacetime curvature and

$$
a=\frac{1}{(D-1)(D-2)}
$$

In addition, we ought to assume that $K$ is a closed homothetic Killing vector (73). In this case we have that

$$
\delta_{\epsilon}(\sqrt{g} R(g))=\epsilon(2-D) \sqrt{g} R(g)+2(D-1) \partial_{\nu}\left(\sqrt{g} g^{\mu \nu} \partial_{\mu} \epsilon\right)
$$

and using the identity

$$
G_{I J} K^{I} \partial_{\nu} \phi^{J}=\frac{1}{D-2} \partial_{\nu}\left(G_{I J} K^{I} K^{J}\right)
$$

one can prove that $\mathcal{L}_{\mathbb{R}}$ transforms into a total derivative.

In order to have a positive definite energy for the gravitational field we must have $G_{I J} K^{I} K^{J}<0$, so one of the scalars is a ghost.

One can fix this gauge invariance by taking

$$
G_{I J} K^{I} K^{J}=-\frac{1}{a \kappa^{2}}
$$

where $\kappa^{2}$ is the gravitational coupling constant. Then the second term of (119) is just the Einstein-Hilbert action and the first term is a sigma model defined now on the surface (121).

\subsection{Kähler manifolds}

We can consider the same kind of model for $n+1$ complex scalar fields $X^{\alpha}$, which are coordinates in a Kähler manifold with metric $G_{\alpha \bar{\beta}}$. We assume now that this metric has a closed homothetic Killing vector $K$. As mentioned at the end of section 4.1, this implies the presence of the holomorphic homothetic Killing vector $H=(1-\mathrm{i} J) K$. The lagrangian density has the form

$$
\mathcal{L}_{\mathbb{C}, 0}=-\sqrt{g} g^{\mu \nu} G_{\alpha \bar{\beta}} \partial_{\mu} X^{\alpha} \partial_{\nu} \bar{X}^{\bar{\beta}}-\frac{1}{4} a G_{\alpha \bar{\beta}} H^{\alpha} \bar{H}^{\bar{\beta}} \sqrt{g} R(g) .
$$

The dilatation symmetry is generated by

$$
K=\frac{1}{2}\left(H^{\alpha}(z) \partial_{\alpha}+\bar{H}^{\bar{\alpha}}(\bar{z}) \partial_{\bar{\alpha}}\right),
$$


but this model has rigid symmetry generated by $J K$, which was not present in the real case. This leads to the infinitesimal transformations

$$
\begin{aligned}
& \delta_{\epsilon} X^{\alpha}=\frac{1}{2} H^{\alpha} \epsilon, \quad \delta_{\varphi} X^{\alpha}=\frac{1}{2} \mathrm{i} H^{\alpha} \varphi \\
& \delta_{\epsilon} g_{\mu \nu}=-2 \epsilon g_{\mu \nu} .
\end{aligned}
$$

Assuming (115), the action is invariant under these transformations where $\epsilon$ can be local, but $\varphi$ is still a global transformation parameter.

A relevant example is the finite transformation

$$
\begin{aligned}
& X^{\alpha} \longmapsto \lambda^{c / 2} X^{\alpha}, \\
& g_{\mu \nu} \longmapsto|\lambda|^{-2} g_{\mu \nu} \quad \lambda=|\lambda| \mathrm{e}^{\mathrm{i} \varphi}=1+\epsilon+\mathrm{i} \varphi+\ldots \in \mathbb{C},
\end{aligned}
$$

for which

$$
H^{\alpha}=c X^{\alpha}, \quad X^{\gamma} \partial_{\gamma} G_{\alpha \bar{\beta}}=0 .
$$

In that case, the transformations (124) can be integrated to a finite transformation. In order to implement the local invariance under $\varphi$, we introduce a $\mathrm{U}(1)$ connection $A_{\mu}$, which transforms as

$$
A_{\mu} \longmapsto A_{\mu}+\partial_{\mu} \varphi
$$

and we couple it minimally to the scalar fields defining

$$
\begin{aligned}
\mathcal{L}_{\mathbb{C}}= & -\sqrt{g} g^{\mu \nu} G_{\alpha \bar{\beta}} D_{\mu} X^{\alpha} D_{\nu} \bar{X}^{\bar{\beta}}-\frac{1}{4} a G_{\alpha \bar{\beta}} H^{\alpha} \bar{H}^{\bar{\beta}} \sqrt{g} R(g) . \\
& D_{\mu} X^{\alpha}=\partial_{\mu} X^{\alpha}-\frac{1}{2} \mathrm{i} A_{\mu} H^{\alpha},
\end{aligned}
$$

For shortness, we denote

$$
N=\frac{1}{c^{2}} G_{\alpha \bar{\beta}} H^{\alpha} \bar{H}^{\bar{\beta}}
$$

Using (77) for the Levi-Civita connection, we have also that

$$
\partial_{\alpha} N=\frac{1}{c} G_{\alpha \bar{\beta}} \bar{H}^{\bar{\beta}}, \quad \partial_{\alpha} \partial_{\bar{\beta}} N=G_{\alpha \bar{\beta}} .
$$

Hence, $N$ is the Kähler potential of the manifold described by the $X^{\alpha}$.

The field equation for the auxiliary field $A_{\mu}$ is algebraic and it allows us to solve for $A_{\mu}$ :

$$
A_{\mu}=\frac{\mathrm{i}}{c^{2} N} G_{\alpha \bar{\beta}}\left(H^{\alpha} \partial_{\mu} \bar{X}^{\bar{\beta}}-\partial_{\mu} X^{\alpha} \bar{H}^{\bar{\beta}}\right)=\frac{\mathrm{i}}{c N}\left(\partial_{\mu} \bar{X}^{\bar{\beta}} \partial_{\bar{\beta}} N-\partial_{\mu} X^{\alpha} \partial_{\alpha} N\right) .
$$


The first term of 127 , the scalar kinetic term $\mathcal{L}_{\text {scalar, }}$, is then

$$
\begin{aligned}
\frac{\mathcal{L}_{\text {scalar }}}{\sqrt{g}}= & -G_{\alpha \bar{\beta}} \partial_{\mu} X^{\alpha} \partial^{\mu} \bar{X}^{\bar{\beta}}-\frac{1}{4 N}\left[\partial_{\mu} \bar{X}^{\bar{\beta}} \partial_{\bar{\beta}} N-\partial_{\mu} X^{\alpha} \partial_{\alpha} N\right]^{2} \\
= & -\partial_{\mu} X^{\alpha} \partial^{\mu} \bar{X}^{\bar{\beta}}\left[\partial_{\alpha} \partial_{\bar{\beta}} N-\frac{1}{N}\left(\partial_{\alpha} N\right)\left(\partial_{\bar{\beta}} N\right)\right] \\
& -\frac{1}{4 N}\left[\partial_{\mu} N \partial^{\mu} N\right] \\
= & -N \partial_{\mu} X^{\alpha} \partial^{\mu} \bar{X}^{\bar{\beta}} \partial_{\alpha} \bar{\partial}_{\bar{\beta}} \log |N|-\frac{1}{4 N}\left[\partial_{\mu} N \partial^{\mu} N\right]
\end{aligned}
$$

Notice that $H^{\alpha}$ is a zero mode of the quantity in square brackets in the second line.

We can fix the dilation gauge freedom (125) by taking as before

$$
N=\frac{1}{c^{2}} G_{\alpha \bar{\beta}} H^{\alpha} \bar{H}^{\bar{\beta}}=-\frac{2}{a c^{2} \kappa^{2}} .
$$

The second term of (127) is then the Einstein-Hilbert action.

As $N$ gets a fixed value, a function of $N$ is not convenient as a Kähler potential for the restricted manifold. We will show now how to construct a Kähler potential, restricting to the case (126).

In that case, we rescale the coordinates $X^{\alpha}$, introducing $\eta^{\alpha}$ by

$$
\begin{aligned}
\Phi_{Y}: \tilde{\mathcal{M}} & \longrightarrow \tilde{\mathcal{M}} \\
\eta^{\alpha} & \longrightarrow X^{\alpha}=\eta^{\alpha} Y(\eta, \bar{\eta}),
\end{aligned}
$$

for an arbitrary function $Y(\eta, \bar{\eta})$. Notice that this map is not holomorphic with respect to $J$. However, it induces a new complex structure on $\tilde{\mathcal{M}}$, denoted as $J^{\prime}$, by the commutativity of the diagram

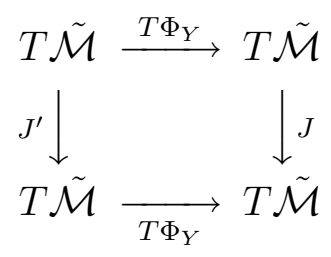

The map $T \Phi_{Y}$ then sends holomorphic vectors with respect to $J^{\prime}$ to holomorphic vectors with respect to $J$. In this sense, it is a holomorphic map. 
Defining

$$
\mathcal{K}=\frac{N}{Y \bar{Y}},
$$

then $\mathcal{K}$ is a function of $(\eta, \bar{\eta})$. The homogeneity properties of $N$ imply

$$
\mathcal{K}(\lambda \eta, \bar{\lambda} \bar{\eta})=\lambda \bar{\lambda} \mathcal{K}(\eta, \bar{\eta}),
$$

and therefore also

$$
\frac{\partial}{\partial X^{\alpha}} N=\bar{Y} \frac{\partial}{\partial \eta^{\alpha}} \mathcal{K}, \quad \frac{\partial^{2}}{\partial X^{\alpha} \partial \bar{X}^{\bar{\beta}}} N=\frac{\partial^{2}}{\partial \eta^{\alpha} \partial \bar{\eta}^{\bar{\beta}}} \mathcal{K} .
$$

Hence, $c \eta^{\alpha} \frac{\partial}{\partial \eta^{\alpha}}$ is a holomorphic homothetic Killing vector with respect to $J^{\prime}$, and $\mathcal{K}$ defines the Kähler potential of a projective Kähler manifold, see Definition 4.2.

The action reduces to

$$
\frac{\mathcal{L}_{\mathbb{C}}}{\sqrt{g}}=-N \partial_{\mu} \eta^{\alpha} \partial^{\mu} \bar{\eta}^{\bar{\beta}} \frac{\partial^{2}}{\partial \eta^{\alpha} \partial \bar{\eta}^{\bar{\beta}}} \log |\mathcal{K}|-\frac{1}{4 N}\left[\partial_{\mu} N \partial^{\mu} N\right]-\frac{1}{4} a c^{2} N R(g) .
$$

The first term in 130 is proportional to $-\tilde{\rho}_{\alpha \bar{\beta}}$ in $(95)$, which is the pull back of a 2 -form on the quotient manifold $\tilde{\rho}=\mathrm{i} \pi^{*} \rho$ as in (96). If $z^{i}$, $i=1, \ldots n$ are coordinates on the quotient, then similarly as in (97), a metric is defined. The appropriate normalization for the Kähler potential is

$$
g_{i \bar{\jmath}}=\partial_{i} \partial_{\bar{\jmath}}\left[-\frac{2}{a c^{2} \kappa^{2}} \log -\frac{a c^{2}}{2} \mathcal{K}\right] .
$$

On the quotient $N$ is constant and thus $\partial_{\mu} N=0$, so the action reduces to a sigma model in dimension $n$ coupled to gravity in the standard way,

$$
\mathcal{L}_{\mathbb{C}}=-\sqrt{g} g_{i \bar{\jmath}} \partial_{\mu} z^{i} \partial^{\mu} \bar{z}^{j}+\frac{1}{\kappa^{2}} \sqrt{g} R(g) .
$$

Note that the $D=4$ values of 115$)$ and $(120)$ lead to $a c^{2} / 2=1 / 3$. That is also the value that one finds in $N=1$ supergravity. For $N=2$ supergravity, one has two scalar manifolds, the one of the vector multiplets, and the one of hypermultiplets. There is another auxiliary field, whose origin is beyond our discussion here, such that when one eliminates the hypermultiplets, the effective value of $a$ is $1 / 2$, i.e. $a c^{2} / 2=1$.

We remark that we need here the lower signs in (97) in order to get the positive kinetic energy for gravity, and the other signatures should all be + in order to have positive kinetic terms of the sigma model. 


\section{Projective special Kähler manifolds}

\subsection{Definition of projective special Kähler manifolds}

A projective special Kähler manifold $\mathcal{M}$ is a Kähler-Hodge manifold such that the manifold $\tilde{\mathcal{M}}$ is rigid special Kähler. The flat connection on $\tilde{\mathcal{M}}$ is an extra structure that also projects to $\mathcal{M}$. Here we have the precise definition.

Definition 6.1 Let $\tilde{\mathcal{M}}$ be a rigid special pseudo-Kähler manifold with complex structure $\tilde{J}$, metric $\tilde{g}$, Kähler form $\tilde{\Phi}$ and flat symplectic connection $\tilde{\nabla}$. We assume that on $\tilde{\mathcal{M}}$ there is a free holomorphic action of $\mathbb{C}^{\times}$such that the fundamental vector field $H$ is a non null, holomorphic homothetic Killing vector for the flat connection,

$$
\begin{aligned}
& \tilde{\nabla}_{Y} H=c Y \quad \forall Y \in T^{1,0} \tilde{\mathcal{M}} \\
& \text { and } \quad \tilde{g}(H, \bar{H}) \neq 0 .
\end{aligned}
$$

Then we say that on $\mathcal{M}=\tilde{\mathcal{M}} / \mathbb{C}^{\times}$there is a projective special Kähler structure.

In fact, we will prove that (131) implies that $H$ is also an holomorphic homothetic Killing vector for the Levi-Civita connection, that is,

$$
\tilde{D}_{Y} H=c Y \quad \text { which is equivalent to } \quad \mathcal{L}_{Y} \tilde{g}=c \tilde{g}, \quad \forall Y \in T^{1,0} \tilde{\mathcal{M}}
$$

so it is enough to require it for the flat connection. Let us look at (131) in special coordinates. From (48) we have

$$
\tilde{\nabla} H=\frac{\partial H^{\beta}}{\partial \eta^{\alpha}} \mathrm{d} \eta^{\alpha} \otimes \frac{\partial}{\partial \eta^{\beta}}-\frac{1}{2} \frac{\partial \tau_{\beta \gamma}}{\partial \eta^{\alpha}} H^{\gamma} \mathrm{d} \eta^{\alpha} \otimes \frac{\partial}{\partial y_{\beta}}=c \mathrm{~d} \eta^{\alpha} \otimes \frac{\partial}{\partial \eta^{\alpha}}
$$

The first term is holomorphic, while in the second there is an holomorphic and an antiholomorphic part, since $y_{\beta}$ is real. To cancel the antiholomorphic part necessarily

$$
\frac{\partial \tau_{\beta \gamma}}{\partial \eta^{\alpha}} H^{\gamma}=0
$$

and then

$$
\frac{\partial H^{\beta}}{\partial \eta^{\alpha}}=c \delta_{\alpha}^{\beta} \quad \Leftrightarrow \quad H^{\beta}=c \eta^{\beta} \text { (up to a constant). }
$$


We can always shift $\eta^{\alpha}$ by a constant, it is still a special coordinate. In particular, (134) implies that special coordinates are homogeneous coordinates as defined in (84). (133) and (134) imply that $\tau_{\alpha \beta}$ are homogeneous functions of $\eta$ of degree 0 ,

$$
\tau_{\alpha \beta}(\lambda \eta)=\tau_{\alpha \beta}(\eta), \quad \lambda \in \mathbb{C}^{\times},
$$

so they depend on the prepotential $\mathcal{F}$ as in 45 :

$$
\tau_{\alpha \beta}=-8 \frac{\partial^{2} \mathcal{F}}{\partial \eta^{\alpha} \partial \eta^{\beta}},
$$

must be an homogeneous function of $\eta$ of degree 2 ,

$$
\mathcal{F}(\lambda \eta)=\lambda^{2} \mathcal{F}(\eta), \quad \lambda \in \mathbb{C}^{\times}
$$

For the Levi-Civita connection, we have

$$
D H=\frac{\partial H^{\beta}}{\partial \eta^{\alpha}} \mathrm{d} \eta^{\alpha} \otimes \frac{\partial}{\partial \eta^{\beta}}+\frac{1}{8} \mathrm{i} g^{\beta \bar{\delta}} \frac{\partial \tau_{\delta \gamma}}{\partial \eta^{\alpha}} H^{\gamma} \mathrm{d} \eta^{\alpha} \otimes \frac{\partial}{\partial \eta_{\beta}},
$$

and using 133 and 134 we get

$$
D H=c \mathrm{~d} \eta^{\alpha} \otimes \frac{\partial}{\partial \eta^{\alpha}}
$$

which proves 132 .

Using (134) we can compute the integral surfaces 80 of $H$ in special coordinates,

$$
H\left(\rho_{\lambda}(x)\right)=\frac{\mathrm{d} \rho_{\lambda}}{\mathrm{d} \lambda} \Leftrightarrow c \rho_{\lambda}^{\alpha}(x)=\frac{\mathrm{d} \rho_{\lambda}^{\alpha}}{\mathrm{d} \lambda} \Leftrightarrow \rho_{\lambda}^{\alpha}(x)=\mathrm{e}^{c \lambda} \eta^{\alpha}(x),
$$

since $\rho_{0}(x)=x$ and $\rho_{0}^{\alpha}(x)=\eta^{\alpha}(x)$.

We will denote also by $\mathfrak{R}_{b}(x)=\rho_{\lambda}(x)$ with $b=\mathrm{e}^{c \lambda} \in \mathbb{C}^{\times}$.

As we saw in general in $(94), \theta=c^{2} \mathcal{K}$.

Since $\nabla$ is flat, it descends to $\mathcal{H}$ as a flat connection, and then it defines a connection on $L \otimes T \mathcal{M}$ as in 109 . This connection is not necessarily flat.

Next we will see that also the holomorphic cubic form descends to an appropriate bundle over $\mathcal{M}$, and we will compute the curvature tensor in terms of it. 
The holomorphic cubic form. We consider the holomorphic cubic form $\Xi$ defined in section 3.7. We want to see how it descends to the manifold $\mathcal{M}$. If $X$ is a vector field on $\mathcal{M}$, its horizontal lift is 106

$$
\tilde{X}=X^{i} \partial_{i}-y \kappa_{i} X^{i} \partial_{y}=X^{i}\left(\partial_{i} \eta^{\alpha}-\kappa_{i} \eta^{\alpha}\right) \partial_{\alpha}
$$

So

$$
\frac{\partial \mathcal{F}}{\partial \eta^{\alpha}} \mathrm{d} \eta^{\alpha}(\tilde{X})=X^{i}\left(\partial_{i} \mathcal{F}-2 \kappa_{i} \mathcal{F}\right)=X^{i} D_{i}^{L}(\mathcal{F})=D_{X}^{L} \mathcal{F}
$$

where we have used the fact that $\mathcal{F}$ is homogeneous of degree 2 . It is in fact a section of $\left(L^{*}\right)^{\otimes 2}$, and $D^{L}$ denotes the covariant derivative with respect to the Hermitian connection. We can also write

$$
D_{i}^{L} \mathcal{F}=\mathrm{e}^{2 \log |\mathcal{K}|} \partial_{i}\left(\mathrm{e}^{-2 \log |\mathcal{K}|} \mathcal{F}\right)=\mathcal{K}^{2} \partial_{i}\left(\frac{1}{\mathcal{K}^{2}} \mathcal{F}\right) .
$$

If $Y, Z$ are also vector fields on $\mathcal{M}$ and $\tilde{Y}, \tilde{Z}$ are their horizontal lifts respectively, we have

$$
\frac{\partial^{3} \mathcal{F}}{\partial \eta^{\alpha} \partial \eta^{\beta} \partial \eta^{\gamma}} \mathrm{d} \eta^{\alpha} \mathrm{d} \eta^{\beta} \mathrm{d} \eta^{\gamma}(\tilde{X}, \tilde{Y}, \tilde{Z})=D_{X}^{L} D_{Y}^{L} D_{Z}^{L}(\mathcal{F})
$$

This shows that the holomorphic cubic form on $\tilde{\mathcal{M}}, \tilde{\Xi}$ descends to a section of $\left(L^{*}\right)^{\otimes 2} \otimes\left(T^{*} \mathcal{M}\right)^{\otimes 3}$,

$$
\Xi(X, Y, Z) \equiv \tilde{\Xi}(\tilde{X}, \tilde{Y}, \tilde{Z}) .
$$

This procedure of lifting the vector fields on $\mathcal{M}$ to $\tilde{\mathcal{M}}$ gives us also another way of computing the metric on $\mathcal{M}$.

The metric. As before, let $\tilde{X}$ and $\tilde{Y}$ be the horizontal lifts of $X$ and $Y$, vector fields on $\mathcal{M}$. Then we have, using (136), (93) and 97),

$$
\begin{aligned}
\tilde{g}(\tilde{X}, \overline{\tilde{Y}}) & =\partial_{\alpha} \partial_{\bar{\beta}} \mathcal{K}\left(\partial_{i} \eta^{\alpha}-\kappa_{i} \eta^{\alpha}\right)\left(\partial_{j} \bar{\eta}^{\beta}-\bar{\kappa}_{j} \bar{\eta}^{\bar{\beta}}\right) X^{i} \bar{Y}^{j} \\
& =\mathcal{K} \partial_{i} \partial_{\bar{\jmath}}(\log |\mathcal{K}|) X^{i} \bar{Y}^{j}=|\mathcal{K}| g(X, \bar{Y}) .
\end{aligned}
$$


The Riemannian curvature on $\mathcal{M}$. From $(109)$ we can compute the curvature tensor of the Levi-Civita connection on $\mathcal{M}$. Since $\tilde{\mathcal{M}}$ and $\mathcal{M}$ are Kähler manifolds we can use (11). We have

$$
R_{i k \bar{\ell}}^{j}=-\partial_{\bar{\ell}} \Gamma_{i k}^{j}=\tilde{R}_{i k \bar{\ell}}^{j}+\partial_{\bar{\ell}}\left(\delta_{i}^{j} \partial_{k} \log |\mathcal{K}|+\delta_{k}^{j} \partial_{i} \log |\mathcal{K}|\right) .
$$

These are the components of the curvature tensor in the coordinates $z^{i}$. To avoid confusion, we will split the coordinates $\eta^{\alpha}$ as $\left(\eta^{0}, \eta^{a}\right), a=1, \ldots n$. In this way the indices $i, j, k$ will always refer to the coordinates $z$.

We can use $\sqrt{62}$ to express $\tilde{R}^{j}{ }_{i k \bar{\ell}}$ in terms of the cubic form $\tilde{\Xi}$. We first have to write $\tilde{\Xi}$ in terms of $\left(y, z^{i}\right)$. We have

$$
\mathrm{d} \eta^{0}=\mathrm{d} y, \quad \mathrm{~d} \eta^{i}=z^{i} \mathrm{~d} y+y \mathrm{~d} z^{i} .
$$

Due to the homogeneity condition 135 and $\tilde{\Xi}$ being a third derivative of $\mathcal{F}$ as in (57), we have

$$
\eta^{\alpha} \tilde{\Xi}_{\alpha \beta \gamma}=0,
$$

and therefore the $\mathrm{d} y$ terms in (139) do not contribute if we rewrite

$$
\tilde{\Xi}=\tilde{\Xi}_{\alpha \beta \gamma} \mathrm{d} \eta^{\alpha} \mathrm{d} \eta^{\beta} \mathrm{d} \eta^{\gamma}=y^{3} \tilde{\Xi}_{a b c} \delta_{i}^{a} \delta_{j}^{b} \delta_{k}^{c} \mathrm{~d} z^{i} \mathrm{~d} z^{j} \mathrm{~d} z^{k}=\Xi_{i j k} \mathrm{~d} z^{i} \mathrm{~d} z^{j} \mathrm{~d} z^{k},
$$

where $\Xi_{i j k}$ has been defined in (137). This leads to

$$
\Xi_{i j k}=2 y^{2} \frac{\partial^{3} \mathcal{F}\left(1, z^{i}\right)}{\partial z^{i} \partial z^{j} \partial z^{k}}
$$

where $\mathcal{F}\left(1, z^{i}\right)$ is $\mathcal{F}(\eta)$ with $\eta^{0}$ replaced by 1 , and $\eta^{i}$ by $z^{i}$.

On the other hand, using $(110)$, we find

$$
R_{i k \bar{\ell}}^{j}=\frac{1}{4 \mathcal{K}^{2}} g^{j j^{\prime}} g^{p^{\prime} \bar{p}} \Xi_{p^{\prime} k i} \bar{\Xi}_{\bar{p} \bar{\jmath}^{\prime} \bar{\ell}} \pm \delta_{i}^{j} g_{k \bar{\ell}} \pm \delta_{k}^{j} g_{i \bar{\ell}} .
$$

Notice that in (141) all the the dependence in $y, \bar{y}$ cancels as $\mathcal{K}$ is proportional to $y \bar{y}$.

\subsection{Examples of projective special Kähler manifolds}

Example 6.2 Projective space and unit ball as special Kähler manifolds. 
We consider the complex projective space $\mathbb{C P}^{m}$ of lines in the complex space $\mathbb{C}^{m+1}$. It is a special case of Example 4.6 , with $p=1, q=m$.

We have a covering of $\mathbb{C P}^{m}$ by open sets

$$
U_{i}=\left\{\text { Lines } S \text { in } \mathbb{C}^{m+1} \text { with }\left.z^{i}\right|_{S} \neq 0\right\},
$$

(these are the lines that do not lie in the hyperplane $z^{i}=0$ ). Let us take a fixed index $i=0$, then we have that

$$
\left.z^{j}\right|_{S}=\left.t^{j} z^{0}\right|_{S}, \quad j \neq 0
$$

so $\left(t^{1}, \ldots, t^{m}\right)$ is a set of coordinates on $U_{0}$.

The tautological bundle is already a line bundle, $L \subset \mathbb{C P}^{m} \times \mathbb{C}^{m+1}$ so there is no need of taking the determinant. On $\mathbb{C P}^{m} \times \mathbb{C}^{m+1}$ we have the fiber metric

$$
\left\langle\zeta, \zeta^{\prime}\right\rangle=\zeta^{0} \bar{\zeta}^{0}+\cdots+\zeta^{m} \bar{\zeta}^{m}
$$

which we will restrict to $L$. On $L$ the fiber metric and the Hermitian connection are

$$
h(t, \bar{t})=1+\sum_{j=1}^{m} t^{j} \bar{t}^{j}, \quad \Gamma_{i}=\left(1+\sum_{j=1}^{m} t^{j} \bar{t}^{j}\right)^{-1} \bar{t}^{i}
$$

The Ricci form 114 becomes

$$
\rho_{i \bar{\jmath}}=-\mathrm{i} g_{i \bar{\jmath}}=-\mathrm{i} \partial_{\bar{\jmath}} \partial_{i} \log h=-\mathrm{i} \partial_{\bar{\jmath}} \partial_{i} \log (1+t \bar{t})=\frac{\mathrm{i}}{h^{2}} \bar{t}^{i} t^{j}-\frac{\mathrm{i}}{h} \delta^{i j} .
$$

We can define the prepotential as

$$
\mathcal{F}=\frac{1}{4} \mathrm{i}\left(\eta^{0} \eta^{0}+\cdots+\eta^{m} \eta^{m}\right), \quad \eta^{i}=t^{i} \eta^{0}, \quad i=1, \ldots m .
$$

Then the Kähler potential in $\tilde{\mathcal{M}}=\mathbb{C}^{m+1}$ and in $\mathbb{C P}^{m}$ is

$$
\mathcal{K}=2 \Im\left(\frac{\partial \mathcal{F}}{\partial \eta} \bar{\eta}\right)=\eta \bar{\eta} ; \quad \log |\mathcal{K}|=\log (1+t \bar{t})+\log \eta^{0} \bar{\eta}^{0}
$$

As the third derivative of the prepotential vanishes, the curvature is given by the last two terms of (141), where we have to use the + signs. 
If we change the fiber metric 142 to

$$
\left\langle\zeta, \zeta^{\prime}\right\rangle=\zeta^{0} \bar{\zeta}^{0}-\cdots-\zeta^{m} \bar{\zeta}^{m},
$$

we obtain that $h$ is positive on the unit ball

$$
h(t, \bar{t})=1-t \bar{t}>0 \quad \text { for } \quad t \bar{t}<1,
$$

which is the symmetric space

$$
\frac{\mathrm{SU}(1, m)}{\mathrm{SU}(m) \times \mathrm{U}(1)}
$$

Notice that the metric is

$$
g_{i \bar{\jmath}}=\frac{1}{h^{2}} t^{i} \bar{t}^{j}+\frac{1}{h} \delta^{i j}=-\partial_{i} \bar{\partial}_{j} \log h .
$$

This means that we have to start with the negative Kähler potential $\mathcal{K}$. This sign is important in physical applications (supergravity), as we saw in section 5. In this case, we have to use the - signs in the last two terms of (141).

Example 6.3 A pseudo-Riemannian special Kähler manifold

We want to describe now the pseudo-Riemannian symmetric space

$$
\frac{\mathrm{SU}(1,2)}{\mathrm{SU}(1,1) \times \mathrm{U}(1)}
$$

We start the construction as for the projective space, on which we try to define a pseudo-Riemannian metric. As we did for the passage to the unit-ball, we will have to restrict to those points where this metric is non degenerate.

As a fiber metric on the trivial bundle $\mathbb{C P}^{2} \times \mathbb{C}^{3}$ we take

$$
\langle\zeta, \zeta\rangle=\bar{\zeta}^{1} \zeta^{1}-\bar{\zeta}^{2} \zeta^{2}+\bar{\zeta}^{3} \zeta^{3}
$$

The space is covered by the three open sets

$$
U_{i}=\left\{\left(\begin{array}{c}
\zeta^{1} \\
\zeta^{2} \\
\zeta^{3}
\end{array}\right) \mid \zeta^{i} \neq 0\right\}, \quad i=1,2,3
$$


as before. In the patch $U_{1}$, we have

$$
\left(\begin{array}{c}
\zeta^{2} \\
\zeta^{3}
\end{array}\right)=T^{1} \zeta^{1}, \quad \text { with } \quad T^{1}=\left(\begin{array}{c}
t^{21} \\
t^{31}
\end{array}\right),
$$

and a local section of the tautological bundle is given by a function $\zeta^{1}\left(T^{1}\right)$,

$$
\zeta\left(T_{1}\right)=\left(\begin{array}{l}
\zeta^{1} \\
\zeta^{2} \\
\zeta^{3}
\end{array}\right)=\left(\begin{array}{c}
1 \\
T^{1}
\end{array}\right) \zeta^{1}\left(T^{1}\right) .
$$

The inner product becomes

$$
\left\langle\zeta\left(T^{1}\right), \zeta^{\prime}\left(T^{1}\right)\right\rangle=\bar{\zeta}^{1}\left(1, T^{1 \dagger}\right) g\left(\begin{array}{c}
1 \\
T^{1}
\end{array}\right) \zeta^{\prime 1}=\bar{\zeta}^{1}\left(1-\vec{t}^{21} t^{21}+\vec{t}^{31} t^{31}\right) \zeta^{\prime 1}
$$

with

$$
g=\left(\begin{array}{ccc}
1 & 0 & 0 \\
0 & -1 & 0 \\
0 & 0 & 1
\end{array}\right)
$$

Doing the same computation for $U_{2}, U_{3}$ we obtain

$$
\begin{array}{ll}
\text { For } U_{1} & \left\langle\zeta\left(T^{1}\right), \zeta^{\prime}\left(T^{1}\right)\right\rangle=\bar{\zeta}^{1}\left(1-\vec{t}^{21} t^{21}+\vec{t}^{31} t^{31}\right) \zeta^{\prime 1} \\
\text { For } U_{2} & \left\langle\zeta\left(T^{2}\right), \zeta^{\prime}\left(T^{2}\right)\right\rangle=\bar{\zeta}^{2}\left(\bar{t}^{12} t^{12}-1+\bar{t}^{32} t^{32}\right) \zeta^{\prime 2} \\
\text { For } U_{3} & \left\langle\zeta\left(T^{3}\right), \zeta^{\prime}\left(T^{3}\right)\right\rangle=\bar{\zeta}^{3}\left(\bar{t}^{13} t^{13}-\bar{t}^{23} t^{23}+1\right) \zeta^{\prime 3}
\end{array}
$$

Equations (145) give us the fiber metric on the tautological (line) bundle. If in each $U_{i}$

$$
\left.\left\langle\zeta, \zeta^{\prime}\right\rangle=h_{i} \zeta^{i} \bar{\zeta}^{\prime i}, \quad \text { (no sum over } i\right)
$$

then

$$
\begin{aligned}
& h_{1}\left(T^{1}\right)=\left(1-\vec{t}^{21} t^{21}+\vec{t}^{31} t^{31}\right) \\
& h_{2}\left(T^{2}\right)=\left(\bar{t}^{12} t^{12}-1+\vec{t}^{32} t^{32}\right) \\
& h_{3}\left(T^{3}\right)=\left(\bar{t}^{13} t^{13}-\vec{t}^{23} t^{23}+1\right) .
\end{aligned}
$$

In the intersections, the change of coordinates

$$
t^{i j}=\frac{1}{t^{j i}}, \quad \zeta^{i}=t^{i j} \zeta^{j}
$$


leaves (146) invariant.

We have to restrict ourselves to the space where the fiber metric is positive definite. Let

$$
\mathcal{U}_{i}=\left\{T^{i} \in U_{i} \mid h_{i}\left(T^{i}\right)>0\right\} .
$$

$\mathcal{U}_{1}$ and $\mathcal{U}_{3}$ are homeomorphic to $\mathbb{C}^{2}$, but $\mathcal{U}_{2}$ is $U_{2}$ minus a ball of radius 1 centered at $T^{2}=0$. The point $T^{2}=0$ is the only point in $U_{2}$ that is not contained in $U_{1}$ or $U_{3}$. So we can safely ignore $\mathcal{U}_{2}$, since $\left\{\mathcal{U}_{1}, \mathcal{U}_{3}\right\}$ form a covering of the space of points where the fiber metric is definite positive.

Notice that in $U_{1}, \xi^{2}=t^{21} \xi^{1}$ serves as a coordinate and the same in $U_{3}$, $\xi^{2}=t^{23} \xi^{3}$, so $\xi^{2}$ is a global coordinate and describes $\mathbb{C}$. The other coordinate, $t^{21}$ or $t^{12}$ respectively in $U_{1}$ and $U_{3}$ describe a sphere $S^{2}$, so we have that the topology of $(143)$ is $S^{2} \times \mathbb{C}$.

Let us compute the Ricci form in $U_{1}$. For simplicity we will denote $t^{21}=$ $t^{2}, t^{31}=t^{3}$.

$$
\rho_{i \bar{\jmath}}=-\mathrm{i} \partial_{i} \partial_{\bar{\jmath}} \log h=\frac{-\mathrm{i}}{h^{2}}\left(\begin{array}{cc}
-1-t^{3} \bar{t}^{3} & t^{3} \bar{t}^{2} \\
t^{2} \bar{t}^{3} & 1-t^{2} \bar{t}^{2}
\end{array}\right)
$$

The metric is

$$
g_{i \bar{\jmath}}=\mathrm{i} \rho_{i \bar{\jmath}}
$$

and it is easy to see that it has one positive and one negative eigenvalue.

The prepotential is

$$
\mathcal{F}=\mathrm{i}\left(\eta^{1} \eta^{1}-\eta^{2} \eta^{2}+\eta^{3} \eta^{3}\right)
$$

with

$$
t^{2}=\frac{\eta^{2}}{\eta^{1}}, \quad t^{3}=\frac{\eta^{3}}{\eta^{1}}
$$

and then the Kähler potential is

$$
\mathcal{K}=4\left(\eta^{1} \bar{\eta}^{1}-\eta^{2} \bar{\eta}^{2}+\eta^{3} \bar{\eta}^{3}\right)=4 \eta_{1} \bar{\eta}_{1} h
$$

We can also see that $\Xi=0$. Then 141 gives $R_{i \bar{\jmath}}=R^{k}{ }_{i k \bar{\jmath}}=3 g_{i \bar{\jmath}}$ and $R=6$. 


\section{Conclusions}

In this paper we have extended the definition of special Kähler geometry to the case of arbitrary signature of the Kähler metric. For the rigid case, we have extended the definition given in Ref. [8], while for the projective case we have given a definition inspired in the conformal calculus framework.

We have seen that the non existence of prepotential in some symplectic coordinates, which was known for projective special geometry [12] (the special geometry that occurs in supergravity), is in fact a characteristic of pseudo Riemannian manifolds, and applies also to the rigid case. This was masked by the fact that in physical applications of rigid special geometry one is only interested in the Euclidean signature, which gives positive definite kinetic energy for the scalar fields.

Projective (or 'local', referring to the local supersymmetry invariance of supergravity) special geometry is obtained from a rigid special manifold that has a closed homothetic Killing vector $K$. If $K$ is such vector and $J$ is the complex structure $J K$ is a Killing vector, so the metric has an extra $U(1)$ symmetry. The result is that the existence of a closed homothetic Killing vector is equivalent to the existence of a holomorphic homothetic Killing vector, which we define in (77). This means essentially that there is an action of the group $\mathbb{C}^{\times}$, and the procedure to obtain the projective special manifold is to take quotient of the rigid 'mother' manifold by this action (and from here, the name of 'projective' geometry). The positive signatures of the kinetic terms of scalars and gravity in supergravity theories require that the rigid manifold has signature $(2,2 n)$. We extend, however, projective special geometry to arbitrary signatures. If the signature of the projective manifold

is $(s, t)$ ( $s$ positive eigenvalues, $t$ negative eigenvalues), then the signature of the 'mother' rigid manifold is either $(s+1, t)$ or $(s, t+1)$. It is the later case that occurs in supergravity. The standard formula for the curvature is generalized to (141), the lower choice in \pm being the standard supergravity case. The other possibility allows us also to discuss special geometries with a compact isometry group.

In fact, this projectivization can be discussed for general Kähler manifolds, not necessarily special. We develop the formalism in this more general case and, for example, we prove that the projective Kähler manifold is automatically Kähler-Hodge. As this is the method that is used in conformal calculus, it implies that all the Kähler manifolds that are constructed in this way for $N=1$ or $N=2$ supergravity satisfy the Kähler-Hodge condition 
that was introduced in Ref. [22]. We also give an interpretation of the LeviCivita connection in these projective Kähler manifolds as induced from the connection of the 'mother' manifold in a particular way, making use of the line bundle and the Hermitian connection. 


\section{A Some technical results}

Lemma A.1 (See lemma A1 in [7]) Let

$$
V=\left(\begin{array}{l}
\alpha \\
\beta
\end{array}\right)
$$

be an $2 n \times n$ matrix of rank $n$ ( $\alpha$ and $\beta$ are $n \times n$ matrices). Then, there is a matrix $S \in \operatorname{Sp}(2 n, \mathbb{R})$ such that the transformed matrix

$$
V^{\prime}=S V=\left(\begin{array}{l}
\alpha^{\prime} \\
\beta^{\prime}
\end{array}\right)
$$

has the property that $\alpha^{\prime}$ itself has rank $n$.

Proof. We make an outline of the proof. Let us denote

$$
\alpha=\left(\begin{array}{c}
\alpha^{1} \\
\vdots \\
\alpha^{n}
\end{array}\right), \quad \beta=\left(\begin{array}{c}
\beta_{1} \\
\vdots \\
\beta_{n}
\end{array}\right),
$$

and let $r-1 \leq n$ be the rank of $\alpha$. If $r-1=n$ we have already the result, so we will take $r-1<n$. Without loosing generality, we can assume that $\alpha^{1}, \ldots \alpha^{r-1}$ are linearly independent. Then

$$
\alpha^{r}=\sum_{i=1}^{r-1} \lambda_{i} \alpha^{i}
$$

Let $\beta_{k}$ be such that $\alpha^{1}, \ldots \alpha^{r-1}, \beta_{k}$ are also linearly independent. For the particular case $k=r$ the symplectic matrix

$$
S=\left(\begin{array}{cc}
\mathbb{1}-E_{r, r} & E_{r, r} \\
-E_{r, r} & \mathbb{1}-E_{r, r}
\end{array}\right)
$$

gives an $\alpha^{\prime}$ with rank $r$.

(We have used the standard notation $\left(E_{i, j}\right)_{k}^{l}=\delta_{i}^{l} \delta_{j k}$. )

In the generic case $k \neq r$, we consider the symplectic matrix

$$
S=\left(\begin{array}{cc}
\mathbb{1}-E_{r, r}-\frac{1}{\sigma} E_{k, r} & E_{r, k}+\sigma E_{r, r} \\
-\frac{1}{\sigma} E_{r, r} & \mathbb{1}-E_{r, r}
\end{array}\right),
$$


where $\sigma$ is a parameter $\sigma \neq 0$. It follows that it is always possible to choose $\sigma$ such that the vectors $\alpha^{\prime 1}, \ldots \alpha^{\prime r}$ are independent. In fact, the conditions on $\sigma$ are that $\sigma$ must be different from some fixed quantities.

By iterating this procedure we see that transforming $V$ with a finite number of symplectic matrices it is possible to construct a matrix $V^{\prime}$ such that $\operatorname{rank}\left(\alpha^{\prime}\right)=n$.

\section{Remark A.2}

When passing from a constant matrix $V$ to a point dependent matrix $V(z)$, one has first to restrict to a neighbourhood where the same components of $V$ are independent (not only in number). Otherwise the theorem could not be applied. So we may have to enlarge the number of open sets of our covering.

Next, we want to consider a constant symplectic transformation in order to have flat Darboux coordinates in each open set. The constraints for $\sigma$ (which must be constant) become now point dependent, namely $\sigma$ must be different from certain functions of $z$ and $\bar{z}$. This is always possible, but perhaps in an open subset of the original open set. For each point there is a neighbourhood contained in a compact set where the constraints can be satisfied. One can cover the manifold with such neighbourhoods and, assuming that the space is locally compact, one can pick up a subcovering which is locally finite.

Lemma A.3 The subgroup of $\operatorname{Sp}(2 n, \mathbb{R})$ formed by the matrices of the form

$$
\left\{\left(\begin{array}{cc}
A & 0 \\
C & \left(A^{T}\right)^{-1}
\end{array}\right)\right\}
$$

is a maximal subgroup.

Before going to the proof let us explain a way of seeing maximality. Let $\mathfrak{g}$ be a Lie algebra and $\mathfrak{s}$ a Lie subalgebra with respective groups $G$ and $S$, with $S \subset G$. We are interested in deciding when $\mathfrak{s}$ is maximal in $\mathfrak{g}$. Note that the adjoint action of $S$ on $\mathfrak{g}$ leaves $\mathfrak{s}$ stable and so $S$ acts on $\mathfrak{a}:=\mathfrak{g} / \mathfrak{s}$.

Theorem A.4 If there is a subgroup $T \subset S$ such that the action of $T$ on $\mathfrak{a}$ is irreducible, then $\mathfrak{s}$ is maximal in $\mathfrak{g}$ 
(We can operate over the complex numbers as maximality over the complexes is stronger than maximality over the reals.)

Proof. Let $\mathfrak{h}$ be a subalgebra such that $\mathfrak{s} \subset \mathfrak{h} \subset \mathfrak{g}$ with $\mathfrak{h} \neq \mathfrak{s}$. We must show that $\mathfrak{h}=\mathfrak{g}$. Since $\mathfrak{h}$ is invariant under $T$, the image $\mathfrak{b}$ of $\mathfrak{h}$ in $\mathfrak{a}$ is stable under $T$. Since $\mathfrak{h}$ is strictly larger than $\mathfrak{s}$, the space $\mathfrak{b}$ is not 0 and is stable under $T$. By the irreducibility of the action of $T$ we must have $\mathfrak{b}=\mathfrak{a}$ so that $\mathfrak{h}=\mathfrak{g}$.

Let us go back to the proof of Lemma A.3.

Proof. In our case $S$ is the lower triangular block group and so we can take $\mathfrak{a}$ to be the space of matrices

$$
\left(\begin{array}{ll}
0 & b \\
0 & 0
\end{array}\right) \quad b=b^{t} .
$$

We take $T$ to be the subgroup

$$
\left(\begin{array}{cc}
A & 0 \\
0 & A^{t^{-1}}
\end{array}\right) \quad A \in \mathrm{GL}(n) .
$$

Then the action of $T$ on $\mathfrak{a}$ works out to be

$$
(A, b) \longmapsto A b A^{t}
$$

which is the representation of GL( $n)$ on the symmetric tensors of the $n$-space, which is known to be irreducible. Applying the Theorem A.4, we complete our proof.

\section{B Connection on a principal bundle and co- variant derivative}

We will relate now the definition 4.3 of connection on a principal bundle to the covariant derivative in associated bundles.

Let $E$ be an associated vector bundle to $P$, with standard fiber $F$, and let $R: G \rightarrow \operatorname{End}(F)$ be the representation of $G$ on $F$. For simplicity we will consider $G \approx R(G)$, although this is not necessary. We want to define the covariant derivative of a section of $E$ in terms of the connection 1-form. Let $\left\{e_{1}, \ldots e_{k}\right\}$ be a basis on $F$. A local frame of $E$ is a set of $k=\operatorname{rank}(E)$ independent local sections of $E$. We will denote it by $\epsilon(m)=\left\{\epsilon_{1}(m), \ldots \epsilon_{k}(m)\right\}$, 
with $m \in \mathcal{M}$. It can be interpreted as an invertible map $\epsilon(m): F \rightarrow E_{m}$ such that

$$
\epsilon(m)\left(e_{a}\right)=\epsilon_{a}(m),
$$

so it provides with an identification of the fiber $E_{m}$ with $F$. The set of frames is a principal bundle with structure group $\mathrm{GL}_{n}$. $P$ is a subbundle of the bundle of frames, so a local section $s: \mathcal{M} \rightarrow P$ is a local frame of $E$.

The pull-back $\Gamma=\sigma^{*} \omega$ defines a local $\mathfrak{g}$-valued 1-form on $\mathcal{M}$. The covariant derivative of a local section of $E, \sigma=\sigma^{a} \epsilon_{a}$, is given by

$$
\nabla_{i} \sigma=\left(\partial_{i} \sigma^{a}+\Gamma_{i b}^{a} \sigma^{b}\right) \epsilon_{a}, \quad \text { so } \quad \Gamma_{i b}^{a}=\left(\nabla_{i} \epsilon_{b}\right)^{a} .
$$

(148) relates the definition of connection as a $\mathfrak{g}$-valued 1-form on $P$ with the notion of covariant derivative that we have been using through the text. (148) is given in terms of a local section $\sigma$ on $P$, but changing the section gives the usual gauge transformation of the local connection 1-form on $\mathcal{M}$. The coordinate independent description of the covariant derivative can be found for example in Ref. [23].

\section{Acknowledgments.}

We are grateful to L. Andrianopoli and S. Ferrara for interesting and very useful discussions.

This work is supported in part by the European Community's Human Potential Programme under contract MRTN-CT-2004-005104 'Constituents, fundamental forces and symmetries of the universe'. The work of A.V.P. is supported in part by the FWO - Vlaanderen, project G.0235.05 and by the Federal Office for Scientific, Technical and Cultural Affairs through the "Interuniversity Attraction Poles Programme - Belgian Science Policy" P5/27.

The work of M. A. Ll. and O. M. has been supported in part by research grants from the Spanish Ministerio de Educación y Ciencia (FIS2005-02761 and EU FEDER funds), the Generalitat Valenciana (ACOMP06/187, GV05/102).

A.V.P. thanks the Universitat de València for hospitality during a visit that initiated this work. M. A. Ll. and O. M. want to thank the Instituut voor Theoretische Fysica, Katholieke Universiteit Leuven for its kind hospitality during part of this work. 


\section{References}

[1] G. Sierra and P. K. Townsend, An introduction to $N=2$ rigid supersymmetry, in Supersymmetry and Supergravity 1983, ed. B. Milewski (World Scientific, Singapore, 1983)

[2] J. Gates, S. James, Superspace formulation of new nonlinear sigma models, Nucl. Phys. B238 (1984) 349

[3] B. de Wit and A. Van Proeyen, Potentials and symmetries of general gauged $N=2$ supergravity - Yang-Mills models, Nucl. Phys. B245 (1984) 89

[4] A. Strominger, Special geometry, Commun. Math. Phys. 133 (1990) $163-180$

[5] L. Castellani, R. D'Auria and S. Ferrara, Special Kähler geometry: an intrinsic formulation from $N=2$ space-time supersymmetry, Phys. Lett. B241 (1990) 57

[6] L. Castellani, R. D'Auria and S. Ferrara, Special geometry without special coordinates, Class. Quant. Grav. 7 (1990) 1767-1790

[7] B. Craps, F. Roose, W. Troost and A. Van Proeyen, What is special Kähler geometry?, Nucl. Phys. B503 (1997) 565-613, hep-th/9703082

[8] D. S. Freed, Special Kähler manifolds, Commun. Math. Phys. 203 (1999) 31-52, hep-th/9712042

[9] V. Cortés, C. Mayer, T. Mohaupt and F. Saueressig, Special geometry of Euclidean supersymmetry. I: Vector multiplets, JHEP 03 (2004) 028, hep-th/0312001

[10] V. Cortés, C. Mayer, T. Mohaupt and F. Saueressig, Special geometry of Euclidean supersymmetry. II: Hypermultiplets and the c-map, JHEP 06 (2005) 025, hep-th/0503094

[11] T. Mohaupt, New developments in special geometry, hep-th/0602171

[12] A. Ceresole, R. D'Auria, S. Ferrara and A. Van Proeyen, Duality transformations in supersymmetric Yang-Mills theories coupled to supergravity, Nucl. Phys. B444 (1995) 92-124, hep-th/9502072 
[13] S. Ferrara, L. Girardello and M. Porrati, Minimal Higgs Branch for the Breaking of Half of the Supersymmetries in $N=2$ Supergravity, Phys.

Lett. B366 (1996) 155-159, hep-th/9510074

[14] G. Lopes Cardoso, B. de Wit, J. Käppeli and T. Mohaupt, Black hole partition functions and duality, JHEP 03 (2006) 074, hep-th/0601108

[15] S. Ferrara and O. Maciá, Real symplectic formulation of local special geometry, Phys. Lett. B637 (2006) 102-106, hep-th/0603111

[16] S. Ferrara and O. Maciá, Observations on the Darboux coordinates for rigid special geometry, JHEP 05 (2006) 008, hep-th/0602262

[17] S. Ferrara, R. Kallosh and A. Strominger, $N=2$ extremal black holes, Phys. Rev. D52 (1995) 5412-5416, hep-th/9508072

[18] A. Strominger, Macroscopic Entropy of $N=2$ Extremal Black Holes, Phys. Lett. B383 (1996) 39-43, hep-th/9602111

[19] S. Ferrara and R. Kallosh, Supersymmetry and attractors, Phys. Rev. D54 (1996) 1514-1524, hep-th/9602136

[20] C. M. Hull, Duality and the signature of space-time, JHEP 11 (1998) 017, hep-th/9807127

[21] S. Ferrara, Spinors, superalgebras and the signature of space-time, hep-th/0101123

[22] E. Witten and J. Bagger, Quantization of Newton's constant in certain supergravity theories, Phys. Lett. B115 (1982) 202

[23] S. Kobayashi and K. Nomizu, Foundations of Differential Geometry. John Wiley and Sons Inc., 1996

[24] A. Newlander and L. Nirenberg, Complex analytic coordinates in complex manifolds, Ann. of Math. 63 (1957) 391-404

[25] S. Kobayashi, Differential Geometry of complex vector bundles. Iwanami Shoten Publishers and Princeton University Press., 1987

[26] S. K. Donaldson and P. B. Kronheimer, The Geometry of Four-Manifolds. Oxford Science Publications, Clarendon Press, 19906 
[27] E. Bergshoeff, S. Cucu, T. de Wit, J. Gheerardyn, R. Halbersma, S. Vandoren and A. Van Proeyen, Superconformal $N=2, D=5$ matter with and without actions, JHEP 10 (2002) 045, hep-th/0205230

[28] O. Griffiths and J. Harris, Principles of algebraic geometry. Wiley, 1978

[29] R. Kallosh, L. Kofman, A. D. Linde and A. Van Proeyen, Superconformal symmetry, supergravity and cosmology, Class. Quant. Grav. 17 (2000) 4269-4338, hep-th/0006179, E: 21 (2004) 5017

[30] A. Van Proeyen, Special geometries, from real to quaternionic, hep-th/0110263, proceedings of the 'Workshop on special geometric structures in string theory', Bonn, 8-11/9/2001; Electronic library of Mathematics: http://www.emis.de/proceedings/SGSST2001/ 


\section{Index}

affine transformation, 38

infinitesimal, 39

almost complex linear connections, 10

almost complex manifold, 7

almost Kähler manifold, 10

antiholomorphic form, 9

Bianchi identities, 14

Christoffel symbols, 13

complete linear connection, 40

complete vector field, 39

complex conjugation, 7

complex Grassmannian, 56

complex manifold, 7

tangent bundle, 16

complex projective space, 14, 69

conformal calculus, 5964

covariant derivative, 77

covariant differential, 18

Darboux coordinates, 4, 10

dilatation symmetry, 60

fiber metric, 16

flat coordinates, 20

flat frame, 20

flat symplectic coordinates, 29

fundamental vector field, 44

Hermitian fiber metric, 15

Hermitian metric, 9

Hermitian symmetric space, 59

Hermitian vector bundle, 17

holomorphic cubic form, $35,38,67$

holomorphic form, 9 holomorphic map, 16

holomorphic prepotential, see prepotential

holomorphic vector bundle, 16

homothetic

closed homothetic Killing vector, 41

holomorphic homothetic Killing vector, 43,45

Killing vectors, 40

transformation, 40

horizontal lift, 52

horizontal projection, 53

horizontal space, 52

hypermultiplet, 64

isometry, 40

Killing vector, 40

Kodaira embedding theorem, 50

Kähler manifold, 11

holonomy, 11

Kähler potential, 12

Kähler-Hodge manifold, 5, 38, 50

leaves of a foliation, 44

Levi-Civita connection, 13

local frame, 77

metric connection, 17

Newlander-Nirenberg theorem, 10

prepotential, 3, 28, 32

principal bundle, 11

connection, 77

projective Kähler manifold, 3859 
definition, 44

projective special Kähler geometry, 3

projective special Kähler manifold, 65

72

curvature, 68

definition, 65

pseudo-Riemannian special Kähler manifold, 4

quasi-Kähler manifold, 10

quasicomplex manifold, 7

quasicomplex structure

torsion, 10

restricted holonomy group, 11

Ricci tensor, 14

rigid special Kähler manifold, 1838

curvature, 37

definition, 19

special coordinates, 3, 4, 29, 32

special Kähler geometry, 3

symplectic bundle, 3

symplectic connection, 19

symplectic manifold, 10

symplectic structure, 10

tautological bundle, 57

unit ball, 68

upper half plane, 31

vector multiplet, 64 